\title{
Microtubule-Associated Proteins and their Essential Roles During Mitosis
}

\author{
Helder Maiato $^{1,2}$, Paula Sampaio ${ }^{1}$ and Claudio E.Sunkel ${ }^{1,3}$
}

${ }^{1}$ Instituto de Biologia Molecular e Celular, Universidade do Porto, Rua do Campo Alegre 823, 4150-180 Porto, Portugal

${ }^{2}$ Laboratory of Cell Regulation, Division of Molecular Medicine, Wadsworth Center, New York State Dept. of Health, Albany, NY 12201, USA

${ }^{3}$ Instituto de Ciências Biomédicas de Abel Salazar, Universidade do Porto, Largo do Prof. Abel Salazar, 4000 Porto, Portugal

Running title: Microtubule-associated proteins in cell division

Author for Correspondence:

C.E.Sunkel, Instituto de Biologia Molecular e Celular

Rua do Campo Alegre 823

4150-180 Porto, Portugal

Tel: +351-22-6074900

Fax: +351-22-6099157

Email: cesunkel@ibmc.up.pt 


\section{Table of Contents}

I. Introduction

II. The mitotic apparatus
A. Microtubules
B. Centrosomes
C. Kinetochores

III. MAPs required for cell division: Identification and Properties
A. Non-motor MAPs
B. Microtubule-based motor proteins

IV. Mitotic spindle organization
A. Assembly of a bipolar spindle
B. Spindle orientation and positioning
C. Balance of forces in spindle architecture and function

V. Microtubule-kinetochore attachment and metaphase chromosome alignment
A. Chromosome capture and poleward movement
B. Chromosome congression
C. Kinetochore-attachment checkpoint

VI. Chromosome segregation
A. Anaphase A
B. Anaphase B

\section{Mitotic exit and cytokinesis}
A. Coordination between karyokinesis and cytokinesis
B. Role of microtubules in the specification and positioning of the cleavage site
C. Organization of the spindle midzone and formation of the cleavage furrow
D. Splitting cells apart

VIII. Concluding remarks

References 


\begin{abstract}
Microtubules play essential roles during mitosis, including chromosome capture, congression, and segregation. In addition, microtubules are also required for successful cytokinesis. At the heart of these processes is the ability of microtubules to do work, a property that derives from their intrinsic dynamic behavior. However, if microtubule dynamics were not properly regulated, it is certain that microtubules alone could not accomplish any of these tasks. In vivo, the regulation of microtubule dynamics is the responsibility of microtubule-associated proteins. Among these, we can distinguish several classes according to their function: (1) promotion and stabilization of microtubule polymerization; (2) destabilization or severance of microtubules; (3) functioning as linkers between various structures; or (4) motility-related functions. Here we discuss how the various properties of microtubule-associated proteins can be used to assemble an efficient mitotic apparatus capable of ensuring the bona fide transmission of the genetic information in animal cells.
\end{abstract}

Key words: Mitosis/Microtubule/Microtubule-associated proteins/Spindle/Kinetochore 


\section{Introduction}

Mitosis is a fundamental biological process that has captured the imagination of researchers for more than a century. During this process, the previously duplicated genome is structurally reorganized into compact chromosomes, each made up of two sister chromatids, which are then equally segregated into daughter cells. Mitosis was first described by Walter Flemming in the late 1870s and consists of two distinct processes: division of the nucleus, or karyokinesis, and division of the cytoplasm, or cytokinesis. Traditionally, the division of the nucleus occurs in five stages: prophase, prometaphase, metaphase, anaphase, and telophase. In prophase, chromatin starts to condense, to form clearly defined chromosomes (Figure 1). By this time, centrosomes, which are the major microtubule-organizing centers (MTOCs) in animals (reviewed by Doxsey, 2001), have already migrated to opposite sides of the nucleus, and a bipolar microtubule-based structure, known as the mitotic spindle, is assembled (Figure 2). This structure is responsible for chromosome segregation during mitosis (reviewed by Wittmann et al., 2001). In vertebrates, the end of prophase and the beginning of prometaphase are marked by nuclear envelope breakdown (NEB). During prometaphase, each chromosome initially orients to one pole of the spindle, a process that is mediated by attachment of microtubules (MTs) to the kinetochore, a protein-based structure located on the surface of the primary constriction of each chromatid. Subsequently, MTs derived mostly from the opposite pole bind the other sister kinetochore, and the chromosome becomes bioriented, moving toward the center of the cell and aligning at the spindle equator during metaphase. Later, during anaphase, sister chromatids separate and migrate toward opposite poles of the spindle. This stage can be subdivided into anaphase A and B. During anaphase A, the two chromatids from each chromosome lose their cohesion, split apart, and move toward opposite poles, while during anaphase $\mathrm{B}$, the spindle usually elongates, thereby increasing the distance between the two poles. Finally, during telophase, each set of chromatids decondenses to form two daughter nuclei, and a cleavage furrow forms between them. This cleavage furrow then contracts and eventually gives rise to the midbody, a structure formed by bundled MTs. This structure participates in the division of the cytoplasm and the formation of the two daughter cells during cytokinesis.

MTs are highly dynamic polymers that alternate between states of growth and shrinkage, a phenomenon known as dynamic instability (Mitchison and Kirschner, 1984a). Modulation of MT's dynamic behavior is thought to be due primarily to association with a broad class of proteins known as Microtubule-Associated Proteins (MAPs). However, the definition of MAPs has changed significantly since the time the first of these proteins were isolated 
(reviewed by Olmsted, 1986). MAPs were originally identified as proteins that co-purified with tubulin through repeated cycles of MT polymerization and depolymerization and were shown to stimulate MT assembly, suggesting a possible role form them in stabilizing the polymer. Subsequently, it was proposed that MAPs be defined as proteins that bind MTs in vivo (Solomon et al., 1979). Since spindle organization and function are now known to be governed by many different types of proteins that associate transiently or constitutively with MTs and can modify their dynamic properties, we have chosen a broader definition for MAPs, to include all of those proteins that are found at least transiently associated with MTs, either in vitro or in vivo, regardless of their functional, regulatory, or motility properties. Of course, we have to be careful, when considering a protein to be a MAP according to this definition, to exclude all of the proteins that may bind to MTs indirectly but yet play no role in modulating MT properties or function.

In this review we will first provide a short description of the major components of the mitotic apparatus and the major mitotic events. We will then focus on the roles of various MAPs as the cell transits from interphase into mitosis (see Table 1 for general refence), including: 1) organization and maintenance of the bipolar spindle; 2) attachment of MTs to kinetochores; 3) chromosome movement; and 4) cleavage-furrow formation during cytokinesis. The results obtained to date reveal that MAPs are essential to coordinately regulate the dynamic properties of MTs and their interactions with various cellular structures so that MTs can effectively be used by the cell to move chromosomes during mitosis.

\section{The mitotic apparatus}

\section{A. Microtubules}

\section{Structure}

MTs are anisotropic polymers of $\alpha$ - and $\beta$-tubulin heterodimer subunits. In cells, these are normally organized into 13 linear protofilaments to form a 25-nm diameter cylindrical structure (Tilney et al., 1973; for reviews see Wade and Hyman, 1997; Nogales, 2001). MT subunits were first purified on the basis of their affinity for colchicine, a natural drug that arrests cells in mitosis (Kiefer et al., 1966; Borisy and Taylor, 1967a; Borisy and Taylor, 1967b; Shelanski and Taylor, 1967; Weisenberg et al., 1968), and later $\beta$-tubulin was shown to hydrolyze GTP (Weisenberg et al., 1976), which is required for the dynamic behavior of MTs. 
In order to better understand the dynamic behavior of MTs, it was first necessary to define their structure. Early cryo-electron microscopy (cryo-EM) studies allowed the determination of the kinetic intermediates of dynamic MTs (Mandelkow and Mandelkow, 1986). Through the use of this technique, and backed up by previous observations, it was observed that MTs elongate through formation of an open, curved tubulin sheet, which, triggered by GTP hydrolysis, closes at a variable rate to form a MT cylinder (Simon and Salmon, 1990; Mandelkow et al., 1991; Chretien et al., 1995). In addition, the sheet length fluctuates as the result of rapid closing (which triggers depolymerization) and opening of the cylinder (when polymerization initiates). The effects of GTP hydrolysis on MT structure have also been confirmed by cryo-EM using slowly hydrolyzable GTP analogues (Hyman et al., 1995; Muller-Reichert et al., 1998). More recently, through the use of Xenopus egg extracts, it was shown that the structural transitions at MT ends correlate with the dynamic properties of MTs in this system (Arnal et al., 2000).

\section{Dynamic properties}

The study of MT dynamics in vitro (reviewed by (Desai and Mitchison, 1997) indicated that, during the steady state (when MT length is constant) there is preferential incorporation of tubulin subunits at one end and loss at the other (Margolis and Wilson, 1978). Thus, a unidirectional flux, or treadmilling, of tubulin subunits from one end of the MT to the other normally takes place (Margolis and Wilson, 1981). These results gained further impact after it was shown that treadmilling also occurs in vivo (Rodionov and Borisy, 1997; for reviews see Waterman-Storer Salmon, 1997; Margolis and Wilson, 1998).

Studies of elongation of individual MTs from isolated centrosomes led to the dynamic instability model of MT behavior, which postulates that a single microtubule never reaches a steady state length, but intead interconverts between states of polymerization and depolymerization as a result of GTP hydrolysis (Mitchison and Kirschner, 1984a; Mitchison and Kirschner, 1984b; Horio and Hotani, 1986). Thus, within a given population of MTs, some will be growing and some shrinking (reviewed by Hyman and Karsenti, 1996).

The intrinsic dynamic properties of MTs are the result of a fast-growing end, known as the plus end, which extends from the MTOC, where the MT slow-growing ends, the so-called minus ends, are anchored (Allen and Borisy, 1974). As a MT grows, a GTP-tubulin dimer is incorporated at the plus end, and the bound GTP is hydrolyzed in an exchangeable GTP binding site on the $\beta$-tubulin subunit during or soon after polymerization (Mitchison, 1993). Since the GTP bound to $\alpha$-tubulin cannot be hydrolyzed, this causes the accessible energy 
form on the MT lattice to be predominantly composed of GDP molecules. The rapid loss of GTP-tubulin subunits and oligomers from the MT end is thought to trigger depolymerization, a phenomenon known as ‘catastrophe’ (Walker et al., 1988). Depolymerizing MTs can also infrequently transit back to the state of elongation, which is known as 'rescue' (Walker et al., 1988).

One way in which to ascertain the role of GTP hydrolysis in MT dynamic instability was through the use of non-hydrolizable or slowly-hydrolyzable GTP analogues ( Hyman et al., 1992; reviewed by Caplow, 1992). These studies led to the conclusion that GTP hydrolysis occurs very rapidly, during or soon after subunit addition, and that a lattice composed mainly of exchangeable GDP molecules is very unstable. Concomitantly, there is increasing evidence that polymerizing MTs are stabilized by special structures that associate with their plus ends, originally postulated to be caps of GTP, or GDP- $\mathrm{P}_{\mathrm{i}}$-tubulin (Carlier and Pantaloni, 1981; Hill and Carlier, 1983; Hill and Chen, 1984; Carlier et al., 1984; Mitchison and Kirschner, 1984a). In this model, the loss of such a GTP-cap would result in a catastrophe, whereas the cap's reformation on a depolymerizing end would result in rescue. Attempts to measure the size of the minimum GTP-cap have established that only a few GTP-containing subunits (corresponding to one to three layers of the lattice) are sufficient to stabilize a growing MT (Walker et al., 1991; Drechsel and Kirschner, 1994; Caplow and Shanks, 1996). The MT properties described above were established mainly by in vitro investigation on the intrinsic behavior of purified tubulin under defined conditions. Nevertheless, a growing body of in vivo evidence has defined physiological MT dynamics and has provided quantitation of the various parameters described previously (Cassimeris et al., 1988; Sammak and Borisy, 1988; Schulze and Kirschner, 1988; Shelden and Wadsworth, 1993; Rusan et al., 2001).

Current models of MT dynamics generally accept that treadmilling and dynamic instability are two intrinsic properties of MTs that are likely to coexist in cells, and may account for the execution of various processes such as kinetochore capture and chromosome movement during mitosis (Farrell et al., 1987; Hotani and Horio, 1988; for reviews see Waterman-Storer and Salmon, 1997; Margolis and Wilson, 1998).

\section{B. Centrosomes}

\section{Structure, duplication, and separation}

The term 'centrosome' was originally coined by Theodor Boveri in 1888 to describe an autonomous permanent organelle of the cell, the dynamic center of the cell, and the coordinator of nuclear and cytoplasmic division (for reviews see Mazia, 1984; Doxsey, 2001). As the major MTOC (Figure 3) in animal cells (Pickett-Heaps, 1969), the centrosome 
determines the numbers and the distribution of MTs (Kellogg et al., 1994). The functionally equivalent organelle in yeast is the spindle pole body (SPB) (for reviews see Winey and Byers, 1993; Francis and Davis, 2000; Hagan and Petersen, 2000. In animal cells, the centrosome is composed of two centrioles, which are open-ended cylinders, each comprising nine sets of triplet MTs linked together, plus some surrounding pericentriolar material (reviewed by Doxsey, 2001). Despite its apparent organizational simplicity, the centrosome is an extremely complex organelle and is thought to contain over 100 different proteins (reviewed by Bornens, 2002).

In vertebrate cells, the centrosome duplicates during $S$ phase, and the mother and daughter centrosomes typically separate during late G2 or prophase, before NEB at prometaphase. How centrosome duplication is regulated has remained an intriguing mystery for more than a century. However, recent studies in Xenopus egg extracts revealed that cyclin E and its associated cyclin-dependent kinase, Cdk2, regulate the splitting of centrioles, one of the earliest stages in centrosome duplication (Hinchcliffe et al., 1999; Lacey et al., 1999). Cdk2 seems also to be required for centrosome duplication in mammalian cells, but cyclin A activity predominates over that of cyclin E (Matsumoto et al., 1999; Meraldi et al., 1999).

For a long time it was thought that centrosome separation involved pushing forces generated between interacting MTs of opposite polarities, derived from opposing centrosomes (reviewed by Mazia, 1987). However, more recent studies in newt lung cells suggest that the force-producing mechanism for centrosome separation during spindle formation at least in very large and flat cells is intrinsic to each aster and is not mediated by MT-MT interactions between opposing asters (Waters et al., 1993). In support of these observations, evidence from various model systems has confirmed that motor proteins such as dynein and kinesin-like proteins, localized to the cortex and MT plus-ends, are implicated in this process and may provide both the pulling and the pushing forces required for aster separation (Heck et al., 1993; Blangy et al., 1995; Sharp et al., 1999b; Sharp et al., 2000a).

\section{Role in microtubule nucleation}

Centrosomes are the main sites of MT nucleation in animal cells. MT nucleation occurs in the pericentriolar material and requires a ring-shaped multiprotein complex containing $\gamma$ tubulin, a protein related to the $\alpha$ - and $\beta$-tubulins (Oakley and Oakley, 1989; Moritz et al., 1995; Zheng et al., 1995; reviewed by Gunawardane et al., 2000;). These $\gamma$-tubulin ring complexes ( $\gamma$ TuRCs) form a lattice structure through association of $\gamma$-tubulin with pericentrin, a component of the pericentriolar material (Doxsey et al., 1994; Dictenberg et al., 1998). 
Recent studies on the detailed structure and function of the $\gamma$ TuRCs provided experimental support for a model in which several small $\gamma$-tubulin sub-complexes are organized into a ring by other members of the ring complex, and may function as MT minus-end caps (Keating and Borisy, 2000; Moritz et al., 2000; Wiese and Zheng, 2000). This model postulates that $\alpha$ and $\beta$-tubulin heterodimers bind longitudinally to $\gamma$ TuRCs, creating a layer at which additional dimers bind, and where a MT can form. This model also explains how MT polarity is established by the centrosome, and assumes that $\alpha$-tubulin is the subunit at the minus end and $\beta$-tubulin is the exposed subunit at the plus-end, a geometry which was later confirmed experimentally (Nogales et al., 1999). An alternative model postulates that lateral interactions between $\gamma$-tubulin and both $\alpha$ - and $\beta$-tubulin serve to initiate the assembly of a protofilament, with which other tubulin dimers interact laterally, to eventually form a MT (Erickson and Stoffler, 1996; Erickson, 2000).

\section{Role in spindle organization}

The interphase cytoskeleton is a highly complex multiprotein and multipolymer matrix. This matrix contains an array of polar MTs that is disassembled just before prophase and is replaced during mitosis by a very different and more dynamic structure, the mitotic spindle (for reviews see Schrader, 1944; Mazia, 1961). This structure is composed mainly of centrosome-nucleated MTs (Figure 3) and is regulated by MAPs that appear to mediate all the events that occur during mitosis. Historically, centrosomes have been considered essential for spindle assembly, due to their association with spindle poles and their MT-nucleating activity. However, higher plants, as well as some Drosophila cell lines, lack canonical centrioles but can still organize normal spindles and undergo cell division (Debec et al., 1995; Smirnova and Bajer, 1992). In addition, early laser ablation experiments of centrioles in mammalian cells suggested that mitosis can progress normally without centrioles (Berns and Richardson, 1977). Moreover, in many systems, including Drosophila female meiosis, no centrosomes are found at the spindle poles (for reviews see Wilson, 1925; Gonzalez et al., 1998). These observations suggest that alternative mechanisms exist that ensures spindle bipolarity. Studies using Xenopus egg extracts supported these conclusions (Sawin and Mitchison, 1991a). Further, it was shown that addition of DNA-coated beads to Xenopus egg extracts is sufficient to generate apparently normal bipolar spindles in the complete absence of centrosomes (Heald et al., 1996). Recent genetic studies and other experiments involving a combination of in vivo GFP labeling of centrosomal components, laser microsurgery, and state-of-the-art microscopic techniques, have conclusively demonstrated that such alternative 
pathways also exist in somatic cells, from Drosophila to vertebrates (Bonaccorsi et al., 2000; Khodjakov et al., 2000). In these cases, spindle assembly is likely directed by chromatin/kinetochore-MT interactions, MT-MT interactions, and the combined activities of molecular motors (reviewed by Karsenti and Vernos, 2001). However, it remains unclear how MTs nucleate and organize into kinetochore fibers (k-fibers), for example, in the absence of centrosomes. Together, the above observations strongly suggest that in vertebrates and other higher animals, the role of the centrosome as the organizer of the mitotic spindle is redundant with other mechanisms. Instead, the centrosome may have at least one other vital function in the cell that is independent of its role as a MTOC. Such a function may be related to cell-cycle progression (see discussion ahead and the review by Rieder et al., 2001).

\section{Kinetochores}

\section{Structure}

It was initially observed by Metzner (Metzner, 1894; see also Schrader, 1944) that spindle fibers interact with a specific chromosomal structure, for which the consensual name 'kinetochore' was later proposed by J. A. Moore, in order to ascribe to this structure the functional properties responsible for chromosome movement (Sharp, 1934). The kinetochore is a protein-based structure located on the surface of each chromosome that mediates its interaction with MTs. At the ultrastructural level, the mammalian kinetochore appears as a trilaminar disk located at the primary constriction of each chromosome, the centromere (Brinkley and Stubblefield, 1966; Jokelainen, 1967; for reviews see Rieder, 1982; Craig et al., 1999). Mammalian kinetochores have four morphologically distinct layers, although only three of these can be detected in the presence of MTs (see Earnshaw, 1994). The innermost layer is a disk of densely packed material called the inner plate, and is continuous with the surface of the centromeric heterochromatin underneath the kinetochore. This region is thought to be responsible for the assembly and size determination of a robust kinetochore capable of withstanding the stresses imposed by the spindle. The most consistently observed feature of the kinetochore is the outer plate, a dense structure that is typically $0.5 \mu \mathrm{m}$ in diameter and 30-40 nm thick. The outer plate forms the primary point of attachment for spindle MTs. Between the two plates there is a third layer, more appropriately considered as an interstitial space. This space is a region of 15-35 nm across that does not stain under standard protocols for electron microscopy, and is traversed by numerous fibers that appear to connect the inner and outer regions of the kinetochore (McEwen et al., 1993). The outer surface of the kinetochore is associated with a poorly defined 10-20 nm filamentous 
meshwork known as the fibrous corona. This structure can only be seen in kinetochores that do not have large numbers of associated MTs, and, based on the localization of distinct molecular components, an outer kinetochore corona domain has been proposed (Maiato et al., 2003a). An exhaustive review on the molecular components of the vertebrate kinetochore has been published (Maney et al., 2000), but the list continues to grow rapidly.

\section{Role in microtubule attachment}

Mazia (1961) provided a simple and concise functional description of the kinetochore, as the point of attachment of the chromosome to the mitotic apparatus, or the anchor point for a spindle fiber. Chromosome fragments lacking kinetochores are incapable of mitotic movements, and chromosomes that have lost portions of their arms but retain their kinetochores perform normally (Rieder et al., 1986; Bajer and Vantard, 1988). Thus, the kinetochore was considered for many years to be the only essential part of the chromosome during mitosis. Indeed, kinetochores that were experimentally detached from mammalian centromeres could still interact with spindle MTs and could complete all of the mitotic movements in the absence of other chromosomal components (Brinkley et al., 1988). On the other hand, it seems that several aspects of kinetochore function are related to the behavior of the ends of dynamic MTs to which it is attached (Mitchison, 1988).

As cells enter mitosis, centrosomes nucleate a large number of highly dynamic MTs. It is thought that their plus ends continuously probe the cytoplasmic space, in what has been described as a "search-and-capture” mechanism (Hill, 1985; Kirschner and Mitchison, 1986; Holy and Leibler, 1994). This mechanism proposed that those MTs growing from the centrosome that accidentally contact a kinetochore will become capped and stabilized, while the MTs that do not contact it will soon depolymerize. This idea found strong support when studies in live cells directly showed that dynamically unstable MTs growing from centrosomes become stabilized upon contact with kinetochores (Hayden et al., 1990). Previously, it had been shown that kinetochores of isolated chromosomes could capture the ends of MTs in vitro (Mitchison and Kirschner, 1985). Moreover, kinetochore MTs turn over less rapidly than do astral MTs in vivo (Mitchison et al., 1986), and detachment of the chromosome from the MTs by a microneedle leads to rapid MT depolymerization (Nicklas and Kubai, 1985). However, MT plus ends captured by the kinetochore can still depolymerize while remaining attached (Huitorel and Kirschner, 1988; Koshland et al., 1988; Cassimeris and Salmon, 1991; Hunt and McIntosh, 1998;), and the rate at which attached MT ends transit from growing to shrinking is increased (Hyman and Mitchison, 1990). Thus, a captured MT is stabilized at a kinetochore not because it cannot undergo catastrophe, but 
instead because it cannot detach (Hyman and Karsenti, 1996). In most eukaryotic cells, kinetochores bind a number of MTs resulting in the formation of a k-fiber (Witt et al., 1981). MTs within a k-fiber display coordinated dynamic properties: when the attached kinetochore is moving away from the spindle pole, all MTs grow, whereas and while it is moving toward the pole, all MTs depolymerize (Skibbens et al., 1993).

\section{MAPs required for cell division: identification and properties}

Since the pioneering isolation of the mitotic apparatus from sea urchin eggs (Mazia and Dan, 1952), attempts have been made to identify the proteins that constitute the mitotic spindle. Early reports estimated that tubulin (Mohri, 1968), constitutes only 5-15\% of the total protein fraction present in the spindle (excellent reviews on the initial characterization of MTs and isolation of the mitotic apparatus were compiled by Olmsted and Borisy, 1973 and Sakai, 1978). This important observation led to further investigation directed at unraveling the unknown spindle proteins that are associated with tubulin. Soon after the first description of MT polymerization in vitro (Borisy and Olmsted, 1972; Weisenberg, 1972), it was found that several proteins co-polymerized with brain tubulin, even after several cycles of assembly and disassembly; these were originally described as high-molecular-weight MAPs (Borisy et al., 1975; Murphy and Borisy, 1975). Many of these proteins were later identified, through immunofluorescence with specific antibodies, to be part of the mitotic spindle (Connolly et al., 1978; Sherline and Schiavone, 1978). Through the use of identical biochemical strategies, several other MAPs were identified as components of the mitotic spindle (Bulinski and Borisy, 1980a; Bulinski and Borisy, 1980b; Bulinski and Borisy, 1980c; De Brabander et al., 1981; Izant et al., 1982; Keller and Rebhun, 1982; Zieve, 1982; Vallee and Bloom, 1983; Izant et al., 1983; Scholey et al., 1984; Hirokawa et al., 1985). In general, MAPs are known to be involved in regulation of the dynamic properties of MTs. Some promote MT polymerization and stability while others induce depolymerization. This broad range of funtions suggests that it is the coordinated action of MAPs that governs the assembly and properties of the mitotic spindle (Figure 4). In the following sections we will concentrate on the most recent advances in the molecular characterization of the MAPs that have major implications for mitosis.

\section{A. Non-motor MAPs}

Despite significant advances in the biochemical characterization, the isolation of genes encoding for MAPs started relatively recently. The first report of the cloning of a gene encoding a MAP as a component of the mitotic spindle was in Drosophila (Goldstein et al., 
1986). The authors described the molecular characterization of a non-essential 205-KDa MAP, isolated from Drosophila S2 tissue culture cells. This protein is likely to be the orthologue of MAP4, a widely distributed mammalian structural MAP that is also important for chromosome movement during mitosis (Shiina and Tsukita, 1999).

\section{Stabilizing factors}

a. MAP4. MAP4 was identified as a human protein that stimulated the polymerization of pure tubulin (Bulinski and Borisy, 1980b). Proteins related to MAP4 have also been found in Xenopus (XMAP230) (Andersen et al., 1994; Shiina and Tsukita, 1999) and Drosophila (DMAP205) (Goldstein et al., 1986). In tissue culture cells, MAP4 localizes along interphase and mitotic MTs (Bulinski and Borisy, 1980b; Olson et al., 1995). Studies in vitro indicated that MAP4 stabilizes MTs by specifically promoting rescue events (Ookata et al., 1995). This activity was also shown in vivo, since reduction of MAP4 in culture cells resulted in an overall decrease in tubulin polymer and a slowing of MT growth (Nguyen et al., 1999). MAP4 is phosphorylated by Cdk1 upon the cell's entry in mitosis, without alterion of its ability to bind MTs (Vandre et al., 1991; Tombes et al., 1991; Shiina et al., 1992; Ookata et al., 1997). However, in vitro and in vivo studies have shown that phosphorylation of MAP4 reduces the protein's binding affinity for MTs and completely abolishes its MT-stabilizing activity (Andersen et al., 1994; Ookata et al., 1995; Shiina and Tsukita, 1999; Chang et al., 2001). Further, overexpression of mutant forms of MAP4, which mimic phosphorylation, alters the protein's MT-stabilization activity and causes perturbations in the cell's progression into and through mitosis (Chang et al., 2001). The cell-cycle regulation of MAP4 by phosphorylation suggested that this could be one of the interphase MT-stabilizing factors that must be turned off, in order that MT dynamics be modified at the onset of mitosis; surprisingly, however, MAP4 does not appear to be required during mitosis (reviewed by McNally, 1996). Indeed, microinjection of inactivating antibodies into human fibroblasts and monkey epithelial cells abolishes MAP4 binding to MTs, but cell viability is not affected (Wang et al., 1996). While a mitotic function of MAP4 has not been widely supported by most experimental data, recent results showed that overexpression of the protein causes formation of monopolar spindles, suggesting that this MAP may, indeed, play a role during early stages of mitotic spindle formation (Holmfeldt et al., 2003).

The Xenopus homologue of MAP4 is thought to be XMAP230, however, several studies have highlighted a number of functional differences between the two proteins in their respective systems. XMAP230 localizes to interphase MTs and dissociates from them during early stages of mitosis, but it then re-associates with the spindle during metaphase and 
anaphase (Andersen et al., 1994). Moreover, rather than promoting rescue events, XMAP230 was shown to increase the MT elongation rate, and to strongly suppress catastrophes (Andersen et al., 1994). However, similarly to MAP4, the activity of XMAP230 was shown to be regulated by phosphorylation and overexpression of non-phosphorylatable forms in tissue culture cells affects chromosome separation during anaphase A (Shiina and Tsukita, 1999). Furthermore, XMAP230 was shown to be required for mitotic spindle assembly in vitro and in vivo (Cha et al., 1998; Cha et al., 1999), indicating that XMAP230 does have a mitotic function in this system.

b. CLIP-170. Analysis of MT dynamics in vivo implied that the interaction of MT plusends with various factors or structures affects MT plus-end behavior. Some of these factors have been recently characterized, and are collectively termed MT-plus-end-tracking proteins (+TIPs). They represent a class of highly conserved MAPs that localize to the growing MT plus ends and play an important role during mitosis (reviewed by Schuyler and Pellman, 2001a). CLIP-170 was the first member of this growing family of proteins to be isolated. This protein was shown to localize in small patches close to MT ends (Rickard and Kreis, 1990), and to be required for linking endocytic vesicles to MTs in vitro (Pierre et al., 1992). Vertebrate CLIPs include the brain-specific CLIP-115 (De Zeeuw et al., 1997), and orthologues have been found in several other species, e.g., D-CLIP-190 in Drosophila (Lantz and Miller, 1998), Bik1 in budding yeast (Berlin et al., 1990), and Tip1 in fission yeast (Brunner and Nurse, 2000).

Fluorescence time-lapse microscopy of living cells revealed that GFP-CLIP-170 localizes specifically to growing MT plus ends, and when MTs shrink, or when their dynamic behavior is suppressed by drug treatment, GFP-CLIP-170 rapidly dissociates (Perez et al., 1999). Based on this behavior, it was proposed that GFP-CLIP-170 passively treadmills from the growing MT plus-end, rather than being actively translocated by a motor protein along the elongating tubulin polymer. CLIP-170 targets MT plus-ends either by recognition of a specific transient conformation at the growing tip, or else by co-polymerization with free tubulin. Due to its higher affinity for the GTP-tubulin conformation, CLIP-170 may incorporate into or bind specifically to a cap of GTP-tubulin present at the extremity of a polymerizing MT. Recent evidence from crosslinking and sedimentation velocity experiments favors the co-polymerization hypothesis (Diamantopoulos et al., 1999), but a definitive proof that holds true for living cells is still lacking.

Originally, it was proposed that CLIP-170 plays a role in regulating MT dynamics at the plus-ends (Rickard and Kreis, 1990). While direct evidence for such a role has not yet been 
obtained, experiments in fission yeast have shown that Tip1p prevents premature MT catastrophe, allowing MTs to reach the cell cortex (Brunner and Nurse, 2000; reviewed by Sawin, 2000). Accordingly, mutants for the fission yeast tip1 (Mata and Nurse, 1997; Brunner and Nurse, 2000) or its budding-yeast homologue BIK1 (Berlin et al., 1990) have abnormally short MTs. More recently, CLIP-170 was shown to promote MT rescue in vivo (Komarova et al., 2002), indicating that CLIPs act as anti-catastrophe factors by promoting MT plus-end stability. The binding of CLIP170 to MT ends has been proposed to be negatively regulated by a kinase activity associated with MTs (Rickard and Kreis, 1991; Perez et al., 1999; Hoogenraad et al., 2000). On the other hand, a recent study has shown that CLIP-170 binding to MTs in vitro and in vivo can be positively regulated by FKBP12rapamycin-associated protein kinase (Choi et al., 2002).

During mitosis, CLIP-170 localizes to MT plus ends and accumulates strongly in kinetochores in prometaphase, but only weakly in kinetochores of metaphase chromosomes (Dujardin et al., 1998; Lin et al., 2001). Based on this peculiar behavior, it was initially postulated that CLIP-170 plays a role in kinetochore-MT attachment; however, experimental evidence is still lacking. Candidate functional partners for CLIP-170 are cytoplasmic dynein and its positive regulator, dynactin. CLIP-170 co-localizes with dynein and dynactin subunits, both at cortical sites (Busson et al., 1998; Valetti et al., 1999; Vaughan et al., 1999; reviewed by Dujardin and Vallee, 2002) and at the kinetochore (Dujardin et al., 1998). Based on experiments in which dominant-negative forms of CLIP-170 were expressed in tissue-culture cells, a role for these proteins in kinetochore function during metaphase chromosome alignment had been proposed. Apart from this work, there is no direct evidence for a mitotic role of CLIP-170.

c. CLASPs. Recently, a new family of +TIPs, known as CLASPs (CLIP-associated proteins), was identified from a yeast two-hybrid screening with a conserved region of CLIP115 (Akhmanova et al., 2001; reviewed by McNally, 2001). CLASPs are members of the conserved MAST/Orbit family of MAPs (Inoue et al., 2000; Lemos et al., 2000; reviewed by Sharp, 2002). Although CLASPs were isolated on the basis of their interaction with CLIPs, they do not require this interaction to bind MTs (Akhmanova et al., 2001). Indeed, at least CLASP1 is known to have a central MT targeting domain independent of the CLIP-binding domain at its C-terminal (Maiato et al., 2003a).

In polarized interphase fibroblasts, CLASPs localize at the plus ends of MTs, and seem to be responsible for orienting stable MT arrays at the cell's leading edge (Akhmanova et al., 2001). During mitosis (Figure 4), CLASPs show a highly dynamic distribution, concentrating 
at the plus ends of growing MTs, centrosomes, and kinetochores. During anaphase, they accumulate in the spindle midzone and they ultimately concentrate at the midbody matrix (also known as the Flemming body) during telophase and cytokinesis (Lemos et al., 2000; Maiato et al., 2003a). Significantly, CLASP1 localization echoes the distribution of MT plus-ends. Therefore, it is reasonable to suggest that this protein family is responsible for at least some localized regulation of MT dynamics during mitosis.

Surprisingly, MAST/Orbit was shown to bind tubulin in a GTP-dependent manner (Inoue et al., 2000). Moreover, Stu1, the putative homologue of MAST/Orbit and CLASPs in S. cerevisiae, was originally found in a screen for suppressors of cold-sensitive mutants of $\beta$ tubulin (Pasqualone and Huffaker, 1994), and was recently shown to associate directly and specifically with $\beta$-tubulin (Yin et al., 2002). In this context, it is tempting to speculate that these proteins regulate some aspect of MT dynamics at or near the stabilizing GTP-cap at the MT plus-ends.

Genetic analyses of CLASP homologues in yeast and Drosophila indicate that these proteins regulate MT behavior during mitosis. All members of this family studied so far have been shown to be essential for assembly and maintenance of the mitotic spindle (Maiato et al., 2002; Yin et al., 2002). The molecular mechanism for the mitotic role of these proteins is still vague. However, studies on CLASP1 indicate that this protein targets independently to the MT plus-ends and to a novel outer-kinetochore region (Maiato et al., 2003a). Furthermore, CLASP1 was shown to directly regulate the behavior of kinetochore-attached MTs, thus making it a strong candidate as a mediator of their coordinated dynamic properties. Further analysis on how CLASP1 modulates the ability of kinetochore-attached MTs to incorporate and lose tubulin subunits at their plus-ends should provide important clues on this process.

d. APC. Adenomatous polyposis coli (APC) tumor suppressor is a large multidomain protein of $\sim 300$ KDa that is conserved from Drosophila to humans (for reviews see MimoriKiyosue and Tsukita, 2001; Bienz, 2002). Germline mutations in the APC gene are responsible for familial adenomatous polyposis, an autosomal dominant inherited disease, while somatic mutations in APC occur in $~ 80 \%$ of sporadic colorectal tumors (reviewed by Fearnhead et al., 2001). The molecular basis of APC function appears to be dependent on the protein's ability to bind $\beta$-catenin, a protein that functions in cell adhesion and Wnt-based signal transduction pathways (Rubinfeld et al., 1993; Su et al., 1993; reviewed by Peifer and Polakis, 2000). 
APC localizes to the MT cytoskeleton, as well as to the leading edges of migrating epithelial cells (Nathke et al., 1996). Analysis of GFP-tagged APC in living cells has revealed a peculiar dynamic behavior for this protein (Mimori-Kiyosue et al., 2000b; reviewed by Mimori-Kiyosue and Tsukita, 2001). APC-GFP moves continuously along a subset of MTs, toward their distal ends, in an ATP-dependent manner, and it accumulates as granular aggregates at their growing plus ends. When MTs begin to shorten, the APC granules drop off from the MT ends. These results suggest that the movement of APC to the MT tips is mediated by a motor protein (Mimori-Kiyosue and Tsukita, 2003).

Studies in vitro and in vivo suggest a role for APC in stabilizing MT plus-ends (Zumbrunn et al., 2001). In addition, APC is hyperphosphorylated during mitosis, implying that its MT-binding activity is cell cycle-regulated (Bhattacharjee et al., 1996). Biochemical studies have shown that APC can be phosphorylated by Cdk1, PKA, and GSK3 $\beta$, resulting in a decrease in its ability to bind and stabilize MTs (Trzepacz et al., 1997; Zumbrunn et al., 2001). APC is also a high-affinity substrate for the spindle -checkpoint Bub kinases, and it forms a complex with Bub1 and Bub3 (Kaplan et al., 2001); however, the real significance of these interactions is not known. Nevertheless, there is some evidence that APC, like Bub1 and Bub3, localizes to kinetochores, in a MT-dependent manner, and it has been implicated in the assurance of proper chromosome segregation (Fodde et al., 2001; Kaplan et al., 2001). However, these results remain highly controversial, since the kinetochore localization of APC could not be reproduced by others (see review by Mimori-Kiyosue and Tsukita, 2001). Future studies on APC localization using GFP-tagging in living cells may turn out to be critical to resolve this paradox.

e. EB1. EB1 is another highly conserved protein that is likely to be involved in the regulation of MT dynamics and that concentrates at growing MT plus ends (reviewed by Tirnauer and Bierer, 2000). Homologues of EB1 have been found in many organisms, including Bim1p in budding yeast (Schwartz et al., 1997), mal3p in fission yeast (Beinhauer et al., 1997), dEB1 in Drosophila (Lu et al., 2001), and DdEB1 in Dictyostelium (Rehberg and Graf, 2002). EB1 was originally discovered as an APC-interacting protein in a yeast twohybrid screen (Su et al., 1995). In addition, EB1 also associates with components of the dynein-dynactin complex, independently of APC and MTs (Berrueta et al., 1999), through direct binding of EB1 to the dynactin subunit, p150 ${ }^{\text {Glued }}$ (Ligon et al., 2003). Presence of the N-terminal of EB1 is sufficient for this protein's association with MTs, while the APC and 
p150 ${ }^{\text {Glued }}$ bind in a mutually exclusive manner to the C-terminal end of EB1 (Askham et al., 2002).

EB1 proteins localize to centrosomes and along cytoplasmic and spindle MTs in a cometlike fashion that shows a higher concentration at the plus-ends (Schwartz et al., 1997; Berrueta et al., 1998; Morrison et al., 1998; Tirnauer et al., 1999; Mimori-Kiyosue et al., 2000b; Rehberg and Graf, 2002; Rogers et al., 2002). The dynamic behavior of EB1 on the distal ends of MTs was analyzed by expression of a GFP fusion protein (Mimori-Kiyosue et al., 2000b). The results revealed that EB1-GFP concentrates at the growing ends of all cytoplasmatic MTs, and it disappears when MTs start to shorten. More interesting, EB1 binds to MT plus-ends at the kinetochore interface of polymerizing MTs, suggesting that it could modulate their dynamic behavior during mitosis (Tirnauer et al., 2002a). Although EB1 was identified as an APC-interacting protein, it concentrates at the plus-ends of MTs independently of APC (Berrueta et al., 1998; Morrison et al., 1998). Nevertheless, EB1 was shown to be required for normal targeting of APC to MT plus ends (Askham et al., 2000; Mimori-Kiyosue et al., 2000a).

EB1 has been implicated in MT stability, since deletion of Bim1 and Mal3 in yeast results in hypersensitivity to MT-destabilizing agents (Beinhauer et al., 1997; Schwartz et al., 1997), and since overexpression of human EB1 induces the formation of stable MT bundles (Bu and Su, 2001; Ligon et al., 2003). It is apparent, from in vitro assays, that EB1 increases the frequency of MT rescue and decreases catastrophes, thus promoting MT elongation (Tirnauer et al., 2002b; Ligon et al., 2003). Moreover, EB1 promotion of MT elongation in vitro requires the presence of the C-terminal half of APC, which is essential for EB1 binding (Nakamura et al., 2001). Furthermore, EB1 seems not only to increase MT stability, but also to promote MT plus-end dynamics during interphase, as shown by studies in yeast and Drosphila cells (Tirnauer et al., 1999; Rogers et al., 2002). The MT stabilizing activity of EB1 is likely to be cell cycle-regulated, since the binding capacity of EB1 along the MT wall is reduced in mitotic extracts, when relative compared to interphase extracts (Tirnauer et al., 2002b). Nevertheless, the comet-like accumulation of EB1 at the plus-ends is not affected.

How EB1 function is regulated remains unclear, but it could be via the interaction with the protein's binding partners. Several studies have shown that APC-EB1 interaction is down-regulated during mitosis, due to APC hyperphosphorylation (Askham et al., 2000; Bhattacharjee et al., 1996; Nakamura et al., 2001). Accordingly, immunoprecipitation studies have indicated that the interaction between EB1 and APC does not appear to occur during mitosis (Askham et al., 2000). 
The properties and the localization of EB1 suggest that it should play a role in mitosis. In support of this, deletion of Bim1 and Mal3 in yeast results in aberrant spindle behavior and chromosome mis-segregation (Beinhauer et al., 1997; Schwartz et al., 1997). Also, a null mutant of DdEB1, as well as depletion of EB1 from Drosophila cells by RNAi, confirmed that the protein is required for normal spindle assembly during mitosis (Rehberg and Graf, 2002; Rogers et al., 2002). Furthermore, depletion of EB1 reduces MT length in mitotic Xenopus egg extracts, but not in interphase extracts (Tirnauer et al., 2002b). Therefore, one possibility is that EB1 performs different roles during interphase and during mitosis, depending on the binding activities of specific partners such as APC and dynactin.

\section{Destabilizing factors}

The high frequency of MT catastrophes observed in vivo suggests the existence of factors that mediate this process (for reviews see McNally, 1999; Walczak, 2000). Such factors oppose the action of stabilizing MAPs and promote MT destabilization, by reducing net tubulin assembly.

a. Katanin. The first MT-destabilizing factor to be discovered was katanin, a MTstimulated ATPase that requires ATP hydrolysis to sever MTs (Vale, 1991; McNally and Vale, 1993; Hartman et al., 1998; Hartman and Vale, 1999). Katanin is a distinct type of enzyme that utilizes energy from nucleotide hydrolysis to mediate MT severance (for reviews see Quarmby and Lohret, 1999; Quarmby, 2000). Katanin is a heterodimer composed of two subunits, a p60 subunit with ATPase activity, and a p80 subunit containing WD40 repeats. The latter subunit targets the enzyme to the centrosome and regulates the MT-severing activity of p60 (Hartman et al., 1998; McNally et al., 2000). Katanin accumulation at centrosomes and spindle poles during mitosis is MT-dependent (McNally et al., 1996; McNally and Thomas, 1998; McNally et al., 2000) and the protein’s mechanism of action may involve recognition of defects in the MT lattice (Davis et al., 2002).

The regulation of katanin is not well understood and seems to be invoked at several levels. Studies in vitro indicate that the protection of MTs by MAPs such as XMAP230 could be via inhibition of katanin activity (McNally et al., 2002). In contrast, Polo-like kinase1 stimulates katanin’s severing activity (McNally et al., 2002).

Katanin is likely to be an important factor in promoting changes in MT dynamics during onset of mitosis. Studies with Xenopus egg extracts have shown that katanin is the major MTsevering factor in mitotic extracts (McNally and Thomas, 1998), whereas this activity is suppressed in interphase (McNally et al., 2002). At centrosomes, katanin may also regulate 
the number of free MT ends, since its inhibition prevents the redistribution of $\gamma$-tubulin from the centrosome to the spindle, and slows down nocodazole-induced spindle MT disassembly (Buster et al., 2002). Surprisingly, depletion of katanin activity does not appear to affect cell progression through mitosis, but it has been shown to be essential for the assembly of the meiotic spindle in C. elegans (Srayko et al., 2000).

b. OP18/stathmin. Op18/stathmin, a phosphoprotein that is overexpressed in some human tumors, was also originally purified as a MT-destabilizing factor (Belmont and Mitchison, 1996). However, Op18’s MT-destabilization mechanim is still unknown. Its hypothetical role on MT dynamics in vivo relies on distinct properties identified in vitro (for reviews see Andersen, 2000; Walczak, 2000; Cassimeris, 2002). Op18 was shown both to promote MT catastrophe and to serve as a tubulin-sequestering enzyme, depending on the $\mathrm{pH}$ and the region of the protein involved (Howell et al., 1999). Independent of its sequestration activity, Op18 appears to interact with MT plus-ends and to induce tubulin GTPase activity (Howell et al., 1999; Larsson et al., 1999a; Larsson et al., 1999b; Segerman et al., 2000; Steinmetz et al., 2000). Op18 also appears to modify MT ends, since the addition of Op18 to Xenopus egg extracts increased the number of blunt MT ends (Arnal et al., 2000).

Op18 is negatively regulated by Cdk phosphorylation (Marklund et al., 1996). Further, Polo-like kinase may also regulate Op18 (Budde et al., 2001). Interestingly, during S and M phases, OP18 is hyperphosphorylated (Strahler et al., 1992; Brattsand et al., 1994; Marklund et al., 1994; reviewed by Cassimeris, 2002), leading to its down-regulation, which seems to be essential for G2/M transition and mitosis (Larsson et al., 1997; Larsson et al., 1995; Marklund et al., 1994). In contrast, there is some evidence that dephosphorylation mediated by PP2A also has a regulatory role with respect to OP18 (Tournebize et al., 1997).

Mitotic chromatin was also shown to modulate OP18 activity resulting in MT growth around chromosomes during spindle assembly in vitro (Andersen et al., 1997). Nevertheless, this activity does not appear to be due to a chromatin-associated kinase, but rather through a MT-associated kinase that becomes dominant due to the stabilization of MTs (via a chromatin-dependent RanGTP pathway) around chromatin (Carazo-Salas et al., 1999; Kuntziger et al., 2001).

c. Kin I. A third class of proteins implicated in MT destabilization are the Kin I members of the kinesin superfamily (Desai et al., 1999). For example, the Xenopus XKCM1 and the mammalian MCAK are two orthologues of these MT-destabilizing factors (Wordeman and Mitchison, 1995; Walczak et al., 1996; Kim et al., 1997). Unlike other motors, 
MCAK/XKCM1 do not move along MTs, but instead are thought to couple ATP hydrolysis with the bending of the protofilament at the Mt plus ends, resulting in depolymerization (Desai et al., 1999; Moores et al., 2002).

MCAK/XKCM1 are soluble in the cytoplasm, but a small fraction is found concentrated at the centromeric regions of mitotic chromosomes and at the spindle poles (Walczak et al., 1996; Wordeman and Mitchison, 1995).

In vitro and in vivo studies have shown that XKCM1/MCAK act as catastrophe factors, affecting either the structural or chemical properties at MT ends (Desai and Mitchison, 1997; Maney et al., 1998; Desai et al., 1999; Tournebize et al., 2000; Maney et al., 2001; KlineSmith and Walczak, 2002). Moreover, the binding of the MCAK/XKCM1 monomer to MT ends induces a conformational change in protofilament structure that is followed by ATPdependent Kin I release and MT depolymerization (Desai et al., 1999; Maney et al., 2001; Niederstrasser et al., 2002). The precise molecular mechanism for this depolymerization activity was only recently established, revealing the existence of an ATP-hydrolyzing complex at the MT ends (Hunter et al., 2003; reviewed by Walczak, 2003).

XKCM1 seems to be required for spindle assembly in animal cells, since microinjection of blocking antibodies or overexpression of the protein results in the formation of aberrant spindles (Walczak et al., 1996; Kline-Smith and Walczak, 2002). Recently, an MCAK partner, ICIS, was identified in a search for MAPs that may stimulate MCAK's MTdestabilizing activity (Ohi et al., 2003). ICIS is also required for mitotic spindle formation in Xenopus egg extracts, and depends on MCAK for proper localization at the inner centromeres. The roles of these proteins in mitosis are discussed in a later section.

\section{Dual-function microtubule modulators}

Until recently, all MAPs had been shown to either stabilize or destabilize the MT polymer. However, recent studies have shown that members of the Dis1-Tog family of MAPs not only promote MT growth, but they may also promote MT depolymerization, giving rise to more dynamic MTs (McNally, 2003).

The Dis1-TOG family is widely conserved in nature and has emerged as a group of highly versatile regulators of MT dynamics (reviewed byOhkura et al., 2001). The founding member of this family of MAPs, Dis1, was identified in a cytological screen for coldsensitive mutations that affected chromosome disjunction in S. pombe (Ohkura et al., 1988; Rockmill and Fogel, 1988). The human homologue, ch-TOG, was identified as a gene overexpressed in colonic and hepatic tumors (Charrasse et al., 1995; Charrasse et al., 1998). Subsequently, several other homologues were identified, including the Xenopus XMAP215 
(Gard and Kirschner, 1987; Tournebize et al., 2000), C. elegans Zyg9 (Vanderslice and Hirsh, 1976; Mathews et al., 1999), Stu2p from S. cerevisiae (Wang and Huffaker, 1997), Alp14, a second homologue in S. pombe (Garcia et al., 2001; Nakaseko et al., 2001), Msps in Drosophila (Cullen et al., 1999) and DdCP224 from Dictyostelium (Graf et al., 2000). More recently, a plant homologue, Mor1, was also identified in Arabidopsis (Whittington et al., 2001).

Proteins from this family are characterized by the presence of an N-terminal domain of 200 amino acid residues and a variable number of HEAT repeats thought to be involved in protein-protein interactions (Neuwald and Hirano, 2000). The C-terminal non-repeat regions are responsible for MT binding. Structural analyses of XMAP215 and Stu2p showed that they are thin, elongate molecules that can span up to eight tubulin dimers along a protofilament (Cassimeris et al., 2001; van Breugel et al., 2003).

All Dis1-TOG family members localize to interphase MTs, and they are found throughout the spindle during mitosis. Stu2 associates laterally with MTs (Wang and Huffaker, 1997) and concentrates in regions rich in MT plus-ends (Kosco et al., 2001). Dis1-TOG family proteins are also found associated with centrosomes/SPB throughout the cell cycle (Wang and Huffaker, 1997; Graf et al., 2000; Nakaseko et al., 2001; Garcia et al., 2001). However, during mitosis in somatic cells, chTOG, XMAP215, and Msps only localize to centrosomes (Charrasse et al., 1998; Cullen et al., 1999; Tournebize et al., 2000; Popov et al., 2001). In general, centrosome/SPB localization appears to be independent of MTs, and immunoelectron microscopy revealed that XMAP215 is in the pericentriolar material (Popov et al., 2001). Furthermore, during mitosis, Dis1, Alp14, and Stu2p localize to the kinetochores (Garcia et al., 2001; He et al., 2001; Nakaseko et al., 2001), suggesting that this family of proteins plays a highly diversified role in mitosis.

Several studies support the idea that Dis1-TOG proteins function in MT plus-end stabilization. XMAP215 was first identified from Xenopus egg extracts through its ability to stimulate rapid MT plus-end growth (Gard and Kirschner, 1987). The depletion of XMAP215 in Xenopus egg extracts results in much shorter and more dynamic MTs, a consequence of increased frequency of catastrophe (Tournebize et al., 2000). Also, the human homologue, ch-TOG, has been shown to promote MT assembly both in solution and from nucleation centers (Charrasse et al., 1998), and to be essential for the formation of taxol-induced asters in extracts from human mitotic cells (Dionne et al., 2000). Depletion of ch-TOG causes a significant reduction in MT polymerization that is ATP-dependent, suggesting that the activity of ch-TOG normally counteracts the action of ATP-dependent destabilizing factors 
(Dionne et al., 2000). Furthermore, ch-TOG is thought to bind along the length of individual protofilaments, although it also binds tubulin dimers or oligomers at a region distinct from its MT-binding domain. Ch-TOG was proposed to promote MT growth through stabilization of nascent protofilaments, and to facilitate tubulin addition at plus ends (Spittle et al., 2000). However, studies in vivo and in vitro have complicated this general picture. The initial characterization of XMAP215 revealed that this protein promotes MT turnover, thus supporting a role in the regulation of MT dynamics (Vasquez et al., 1994). In sharp contradiction, genetic analysis in S. cerevisiae has shown in vivo that when levels of Stu2p are reduced, MTs are much less dynamic, with lower frequency of catastrophe (Kosco et al., 2001). Moreover, mitosis in these mutant cells was severely affected, since chromosomes were not able to align at the metaphase plate. More recently, it was shown in vitro and in vivo that Stu2p promotes MT destabilization by inhibiting MT growth rates, resulting in an increase in catastrophes (van Breugel et al., 2003). Since Stup2 was shown to preferentially bind MT plus ends in vitro, this effect may be the result of interference with the process of addition of tubulin at MT plus ends. Similar studies have also shown, again in vitro, that purified XMAP215 acts as a MT-destabilizing factor (Shirasu-Hiza et al., 2003). These studies have raised the possibility that Dis1-TOG MAPs promote either stabilization or destabilization by antagonizing MT pauses. Along this line of thought, a pause - defined as a metastable intermediate state between phases of polymerization and depolymerization - has been reported to occur both in cells and in Xenopus egg extracts (Tran et al., 1997; Tirnauer et al., 2002b). Therefore, antagonization of the pause state would increase MT dynamics, biasing toward either polymerization or depolymerization, depending on what other factors are also modulating the activity of XMAP215 and Stu2p.

Like most MAPs, Dis1-TOG plus-end MT-stabilizing activity is suppressed by Cdk1dependent phosphorylation as cells progress into mitosis (Vasquez et al., 1999). However, phosphorylation of XMAP215 alters neither this protein's capacity to increase rates of MT shortening, nor its ability to bind taxol-polymerized MTs (Vasquez et al., 1999). Genetic analyses and RNAi studies indicated that, during mitosis, these proteins are required for spindle organization, and they may regulate the balance of forces during the metaphaseanaphase transition (Wood et al., 1980; Kemphues et al., 1986; Nabeshima et al., 1995; Wang and Huffaker, 1997; Matthews et al., 1998; Nabeshima et al., 1998; Cullen et al., 1999; Severin et al., 2001; Gergely et al., 2003).

\section{Coordinated actions of MAPs}


The regulation of MT dynamics within cells appears to be dependent on the coordinated actions of multiple MAPs having either opposing or synergistic activities (reviewed by Schuyler and Pellman, 2001a; Andersen and Wittmann, 2002). The use of Xenopus egg extracts to investigate mechanisms that regulate MT dynamics has revealed that XMAP215 modulates the catastrophe frequency of MTs by opposing the MT-destabilizing activity of XKCM1, suggesting that the basic parameters of MT dynamics can be regulated by the balanced activities of MAPs (Tournebize et al., 2000; for reviews see Andersen, 2000; Heald, 2000; Walczak, 2000). Indeed, the essential features of physiological MT dynamics can be reconstituted in vitro by mixing three purified components: tubulin, XMAP215, and XKCM1 (Kinoshita et al., 2001), implying that these proteins regulate MT dynamics in vivo (reviewed by (Kinoshita et al., 2002). In two other independent studies, it was suggested that the MT stabilizer, MAP4/XMAP230, counteracts katanin-mediated MT disassembly (McNally et al., 2002), and suppresses the activity of the catastrophe factor XKCM1 (Holmfeldt et al., 2002). The dual MT-regulatory properties of XMAP215 and Stu2p suggest that these proteins mediate MT dynamics in conjunction with other factors that promote the growth or shrinkage of MT plus-ends (Pearson et al., 2003; Shirasu-Hiza et al., 2003; van Breugel et al., 2003). Accordingly, in a two-hybrid screening, Stu2 interacts with Bim1 and Bik1 (Chen et al., 1998) and studies in Dictyostelium provide some evidence that DdEB1 is present in a complex with DdCP224 (Rehberg and Graf, 2002). However this is unlikely to be a constitutive interaction, since the two proteins only co-localize at the MT ends. Lastly, recent studies have indicated that genetic interactions between Alp14 and Kin I proteins (Klp5/Klp6) are essential to increase MT dynamic behavior, and to allow bipolar spindle formation during prometaphase (Garcia et al., 2002a).

\section{Chromosomal passengers}

Chromosomal passenger proteins associate with chromosomes during prophase, and become increasingly concentrated at the inner centromere region during prometaphase and metaphase. By the time of the metaphase-anaphase transition, passenger proteins abruptly transfer to the central region of the mitotic spindle and to the cell cortex, in the region where the contractile ring will form (for reviews and proper classification see Earnshaw and Bernat, 1991; Adams, 2001; Earnshaw, 2001). Based on this behavior, it was proposed that these transient chromosome-associated proteins play a direct role in chromosome functions in early mitosis, but shift functions during anaphase and telophase, to modulate the role of the cytoskeleton (Earnshaw and Cooke, 1991). 
The first chromosomal passenger protein to be described, INCENP (for Inner Centromere Protein), attracted interest because of its relocalization from the centromeres to the spindle midzone, ending up in the cleavage furrow as the cell goes through mitosis (Cooke et al., 1987).

As shown by knockout experiments, INCENP is an essential protein during mouse development (Cutts et al., 1999). Since INCENP binds directly to tubulin and requires dynamic MTs for targeting to the cleavage furrow, it can be considered as a MAP (Wheatley et al., 2001b). Furthermore, overexpression of certain truncated forms of INCENP causes ectopic bundling of cytoplasmic MTs, suggesting that it has intrinsic MT-modification properties (Mackay et al., 1998).

Another chromosomal passenger protein is Aurora B, a member of the Aurora protein kinase family first identified in D. melanogaster in a search for genes that regulate the structure and function of the mitotic spindle (Glover et al., 1995). Budding yeast contains a single Aurora kinase, Ipl-1p, which was shown to mediate chromosomal events and to have a function in the spindle throughout mitosis (Francisco and Chan, 1994; Francisco et al., 1994). In contrast, metazoans have evolved a multigenic Aurora kinase family, within which the original Aurora protein is now termed Aurora A and is thought to be involved in spindle dynamics, whereas Aurora B is involved in chromosomal events and cytokinesis (for reviews see Bischoff and Plowman, 1999; Giet and Prigent, 1999a; Carmena and Earnshaw, 2003).

A third chromosomal passenger protein, Survivin, is a member of a family of proteins discovered first in baculoviruses, and soon thereafter in mammalian cells, as negative regulators of the apoptotic response (Miller, 1999; Reed and Bischoff, 2000). These proteins, known as IAPs (inhibitor of apoptosis), are characterized by a zinc-binding motif termed the BIR repeat (baculovirus iap repeat). However, the role, if any, of Survivin in apoptotic regulation remains unclear. It was originally reported that human survivin concentrates at spindle poles, binds to spindle MTs throughout mitosis, and associate with MTs in vitro (Li et al., 1998). In contrast, recent studies have shown that Survivin actually exhibits a classic chromosomal-passenger distribution (Skoufias et al., 2000; Wheatley et al., 2001a). This controversy seems to be the result of the properties of the monoclonal antibody originally used to determine the mitotic localization of Survivin (Li et al., 1998). Although this antibody does recognize human Survivin in vitro (Wheatley et al., 2001a), the persistence of spindle staining with this antibody in cells depleted of all known Survivin isoforms by RNAi (Carvalho et al., 2003) strongly suggests that the antibody also recognize an additional epitope. In contrast, the same depletion experiments completely abolished chromosome 
accumulation of Survivin detected either with a polyclonal antibody or in cells stably expressing GFP-Survivin (Carvalho et al., 2003). These results convincingly demonstrate that Survivin is a bona fide chromosomal-passenger protein. Another argument against a role of Survivin in apoptosis was revealed by RNAi-mediated inactivation of BIR1, the Survivin orthologue in C. elegans (Fraser, 1999). Such inactivation leads to defects in cytokinesis, implying that this protein normally functions in cell division rather than cell death.

Accordingly, survivin knockout mice die by 4.5 days due to defects in cell division, and not apoptosis (Uren et al., 2000). A number of recent studies have shown that INCENP is a specific binding partner for Aurora B in several higher eukaryotes (Adams et al., 2000; Kaitna et al., 2000). Moreover, SLI15, the putative orthologue of INCENP in budding yeast, was found to interact genetically with and to be a physical binding partner of Ipl-1 (Kim, 1999). Interference with the function of both proteins by several means leads to dramatic defects in chromosome segregation during mitosis. Consistently, INCENP, Aurora B and Survivin are mutually required for their correct centromere and targeting to the spindle midzone in several organisms (Adams et al., 2000; Kaitna et al., 2000; Speliotes, 2000; Adams et al., 2001; Wheatley et al., 2001a; Wheatley et al., 2001b; Carvalho et al., 2003; Chen et al., 2003; Honda et al., 2003). Moreover, direct interactions among Survivin, Aurora $\mathrm{B}$ and INCENP were shown both in vitro and in cells in which endogenous INCENP localization was disrupted by means of transfection with dominant-negative constructs (Wheatley et al., 2001a). Further, direct evidence for the presence of Survivin in the complex was obtained recently (Bolton et al., 2002; Leverson et al., 2002). In addition to Aurora B, INCENP, and Survivin, a fourth component of this complex was found in C. elegans and was termed CSC-1 (Romano et al., 2003), but significant similarity with any protein in more divergent organisms was not found. Finally, an antigen known as TD-60, which behaves as a typical chromosomal passenger, remained mysterious for more than a decade (Andreassen et al., 1991). TD-60 co-localizes with INCENP and Aurora B throughout mitosis and cytokinesis (Martineau-Thuillier et al., 1998), but it was cloned only recently and was shown to be a member of the RCC1 family required for prometaphase progression (Mollinari et al., 2003). While there is some evidence for INCENP and Survivin binding directly to MTs, the existence of such binding remains undetermined for the other passenger proteins. Maybe this passenger's association with MTs is mediated either by INCENP or Survivin.

\section{B. Microtubule-based motor proteins}

Certain molecular motors are MAPs with the special capability to convert chemical energy in the form of ATP into force and movement (Barton and Goldstein, 1996; Hirokawa 
et al., 1998). Using isolated spindles, Mazia and co-workers (1961) provided the first description of an ATPase activity associated with this structure. Subsequently, a soluble 13S protein fraction with ATPase activity was isolated from sea urchin spindles (Weisenberg, 1968). Its sedimentation coefficient and activity resembled those of ciliary dynein, the first MAP to be identified (Gibbons and Rowe, 1965). Although the idea was very controversial at that time, there was increasing suspicion of the presence and function of dynein in the spindle (McIntosh et al., 1975; Mohri, 1976; Sakai, 1976; reviewed by Sakai, 1978). Through the use of ATP analogs, kinesin was isolated from squid axoplasm and was shown to be involved in MT-based motility of particles (Vale et al., 1985). Subsequently, a protein immunologically related to kinesin was localized in the mitotic spindle (Scholey et al., 1985).

As a consequence of MT polarity, these motors can now be classified as either minus-end directed, with an activity capable of moving cargo from the plus-end to the minus-end of the MT, or plus-end directed, with an activity capable of moving cargo from the minus-end to the plus-end of the MT. Mitotic processes known to be mediated by molecular motors include: separation of spindle poles during G2/prophase and the maintenance of centrosome separation throughout mitosis; movement of chromosomes toward and away from the poles during prometaphase, metaphase, and anaphase; spindle elongation during anaphase B; and finally, assembly and function of the spindle midzone and midbody during telophase and cytokinesis.

\section{Dynein}

Dynein was first discovered in cilia and flagella, where it powers MT sliding in the axoneme by generating minus-end directed MT movement (Gibbons and Rowe, 1965). Cytoplasmic dynein, discovered later to be one of the original high-molecular-weight proteins, MAP1C (Lye et al., 1987; Paschal et al., 1987), is involved in diverse activities including intracellular transport, nuclear migration, and the orientation of the mitotic spindle (for reviews see Karki and Holzbaur, 1999). It consists of a massive multisubunit complex composed of two heavy chains ( $>400 \mathrm{KDa}$ ), each of which folds to form two heads of the motor domain that binds to MTs and hydrolyzes ATP; three or four intermediate chains ( 74 $\mathrm{KDa}$ ); and four light chains ( $55 \mathrm{KDa}$ ), which are thought to bind cargo. Cytoplasmic dynein functions in close cooperation with dynactin, which works as an activating multisubunit complex (for reviews see Schroer, 1994; Vallee and Gee, 1998; Hirokawa et al., 1998; Karki and Holzbaur, 1999).

\section{Kinesins}


Kinesin was first found in neural tissue, where it appears to generate plus-end-directed movements needed for axonal transport (Vale et al., 1985). Structurally, there are three major groups of kinesins, classified according to the position of the motor domain: N-terminal kinesins, C-terminal kinesins, and internal motor domain kinesins (reviewed by Vale and Fletterick, 1997). Both N- and C-terminal kinesin groups are further subdivided according to common structural features. Kinesin motors that do not fall into any of these groups are known as 'orphans'. Conventional kinesins contain a distinct motor domain equivalent to the force-producing element, which often corresponds to the MT-binding domain. Additionally, the C-terminal tail of a kinesin is usually separated from the motor domain by a coiled-coil region termed the stalk, thought to target the motor domain to a particular cargo within the cell (thereby constituting the cargo domain). Subsequent studies have shown that other relatives in the kinesin superfamily have essential functions during cell division. These kinesin-like proteins (KLPs) share a common core motor domain, which is attached to various tail domains thought to target motors to other proteins or cellular structures.

\section{Organization of the mitotic spindle}

During interphase, MTs are relatively long and stable; however, at the G2/M transition, the interphase MT network is disassembled and MTs become highly dynamic, showing high frequencies of both rescue and catastrophe (Belmont et al., 1990; Rusan et al., 2001). The transition from interphase to mitosis is accompanied by an increase in MT dynamics (Saxton et al., 1984; Zhai et al., 1996) and a concomitant decrease in the overall MT polymer level that occurs at the time of NEB. Despite of net negative pressures on MT polymerization, MT polymer levels increase as mitosis progresses, largely as a result of chromosome-MT interaction (Zhai et al., 1996). Increased MT dynamics is associated with phosphorylation of MAPs, rendering them unable to promote MT stabilization and thus allowing the action of destabilizing factors to become dominant (Andersen, 1998). Active cyclin dependent kinase 1 (Cdk1) appears to be a major regulator of this process, since the addition of Cdk1 kinase to interphase Xenopus egg extracts induces an increase in MT dynamics similar to that observed in vivo during the G2/M transition (Verde et al., 1990).

\section{A. Assembly of a bipolar spindle}

\section{Motor proteins}

The roles of multiple MT motors in spindle assembly (see Figure 3) have been the subject of intense investigation (for reviews see Hoyt and Geiser, 1996; Vernos and Karsenti, 1996). Pioneering work in budding yeast showed that disruption of the balance of the kinesin motors 
Cin8, Kip1p, and Kar3p resulted in a rapid collapse of the spindle (Saunders and Hoyt, 1992; Saunders et al., 1997b). Studies on mitotic spindles assembled in vitro using DNA-coated beads incubated in the presence of Xenopus egg extracts have led to a model in which multiple MT-based motor proteins, such as XKLP1 (Vernos et al., 1995); dynein; Eg5, the vertebrate homologue of Drosophila KLP61F (Le Guellec et al., 1991; Heck et al., 1993); and XCTK2, the Xenopus counterpart of Drosophila Ncd (Endow et al., 1990; McDonald et al., 1990), are required for establishing spindle bipolarity (Walczak et al., 1998). Subsequent studies in living Drosophila embryos have confirmed that spindle assembly, maintenance, and elongation in vivo depend upon the coordinated activities of most of these motors (Sharp et al., 1999b; Sharp et al., 2000a).

KLP61F/Eg5 are members of the conserved bimC kinesin family (reviewed by Kashina et al., 1997) shown to be required for bipolar spindle formation (Sawin et al., 1992; Heck et al., 1993; Mayer et al., 1999). The role of Eg5 in spindle formation may be explained, in part, by this protein's association with dynactin, which is regulated in a phosphorylation-dependent manner by Cdk1 (Blangy et al., 1995; Blangy et al., 1997). Eg5 has also been shown to associate with and be phosphorylated in vivo by Eg2, the Xenopus orthologue of Aurora A kinase (Giet et al., 1999), which is also required for the formation of a bipolar spindle (Glover et al., 1995). These all appear to be part of the same molecular machinery. The action of Eg5 and other kinesins like XKLP2 (Boleti et al., 1996) is thought to generate the pushing forces required for centrosome separation. Moreover, the activity of Eg5 can be counteracted by that of HSET (Mountain et al., 1999), a human minus end-directed kinesin-like protein related to yeast Kar3p (Ando et al., 1994; Kuriyama et al., 1995). This finding supports the notion that spindle bipolarity is achieved by the coordinated action of several motor proteins in a highly regulated pathway.

Cytoplasmic dynein and dynactin localize to astral MTs and to cortical sites in mitotic cells, and are thought to anchor astral MTs to the cell cortex (Busson et al., 1998). Likewise, they have been shown to bind the nuclear envelope, where they can stabilize MTs and where they play an essential role in centrosome migration along the nucleus (Busson et al., 1998; Gonczy et al., 1999; Robinson et al., 1999; Piehl and Cassimeris, 2003). Dynein was also proposed to facilitate NEB by moving the nuclear membranes poleward along the astral MTs (Beaudouin et al., 2002; Salina et al., 2002). Nevertheless, it is well known that in cells treated with MT-depolymerizing drugs (and therefore having no MTs), the nuclear envelope breaks down with normal kinetics and the cells accumulate in c-mitosis (reviewed by Rieder and Palazzo, 1992). Dynein also localizes to spindle MTs and kinetochores ( Pfarr et al., 
1990; Steuer et al., 1990; Yoshida et al., 1985; Hays et al., 1994) and through its minus-enddirected motor activity at the cell cortex may provide the pulling forces that maintain separation of spindle poles that were pulled apart during the early stages of mitotic spindle formation (Vaisberg et al., 1993). Additionally, the minus-end directed activity of dynein/dynactin is responsible for accumulation of several proteins on centrosomes and thus has an important role in the organization of spindle poles (reviewed by Merdes and Cleveland, 1997).

\section{Microtubule-associated proteins}

a. NuMA. NuMA was the first non-motor MAP shown to be required for the establishment and maintenance of spindle bipolarity (Yang and Snyder, 1992). NuMA is a large protein that is present in the nucleus of interphase cells and concentrates in the polar regions of the spindle during mitosis (Lydersen and Pettijohn, 1980; reviewed by Compton and Cleveland, 1994). It was proposed that NuMA, together with dynein/dynactin, tethers MTs at the spindle poles and is essential for mitotic spindle pole assembly and stabilization (Gaglio et al., 1995; Merdes et al., 1996; Gaglio et al., 1997; Quintyne et al., 1999; for reviews see Merdes and Cleveland, 1997; Compton, 1998). NuMA is transported to MT minus-ends by the dynein/dynactin complex (Merdes et al., 2000) and forms a matrix at the spindle poles that appears to anchor MT ends through stable crosslinking of MT fibers (Dionne et al., 1999; Haren and Merdes, 2002). The anchorage of MTs at spindle poles by NuMA, together with the action of the motor protein HSET, is essential to generate proper tension across sister kinetochores, and for chromosome movement during mitosis (Gordon et al., 2001).

b. TPX2. Another MAP involved in the organization of spindle poles and assembly of spindle MTs is TPX2 (Gruss et al., 2001; Wittmann et al., 2000). Initially identified as the targeting protein of Xklp2 (Wittmann et al., 1998; Wittmann et al., 2000), TPX2 has been shown in more recent studies to be required for Aurora A kinase activation and localization to the spindle MTs, a process that is stimulated by Ran-GTP (Kufer et al., 2002; Tsai et al., 2003; Eyers et al., 2003). Inhibition of human TPX2 in tissue-culture cells results in fragmentation of spindle poles and the formation of multipolar spindles, through a mechanism dependent on the bipolar kinesin, Eg5 (Garrett et al., 2002; Kufer et al., 2002). Based on these observations it was proposed that hTPX2 is involved in the balance between MT stability and motor-dependent forces required for spindle assembly (Garrett et al., 2002).

c. CLASPs. Members of the CLASP family (Maiato et al., 2002) have also been implicated in the maintenance of spindle architecture (Figure 5). How these proteins 
contribute to this process can be explained by several mechanisms. The yeast counterpart STU1 was shown to functionally interact with a member of the kinesin family, cin8 (Yin et al., 2002). These observations, together with the similarities in the loss-of-function phenotypes of Drosophila mutants for KLP61F, the orthologue of cin8 in this organism (Heck et al., 1993; Sharp et al., 1999a), strongly suggest that MAPs from the CLASP family and kinesin-like motors act cooperatively to maintain spindle bipolarity (reviewed by Sharp, 2002). In addition, it was found that the origin of the monopolar spindles seen after interference with MAST/Orbit function in Drosophila was due to the collapse of a preassembled bipolar spindle, and that chromosomes were unable to congress to the metaphase plate (Maiato et al., 2002). These observations suggest that MAST/Orbit have a role in MTkinetochore attachment or some aspect of the dynamic regulation of kinetochore-attached MTs that, when compromised, contributes directly to the stability of the mitotic spindle and chromosome movement. Indeed, in vivo analysis of human cells injected with anti-CLASP1 antibodies revealed that this protein is able to regulate MT dynamics at the kinetochore and throughout the spindle (Maiato et al., 2003a; Maiato et al., 2003b).

d. EB1. EB1 has also been implicated in spindle assembly and function. In Dictyostelium, null mutants for EB1 show aberrant spindle assembly (Rehberg and Graf, 2002). Mutaiton in bim1 in yeast and depletion of EB1 in Drosophila by RNAi also result in short spindles and short astral MTs (Tirnauer et al., 1999; Rogers et al., 2002). In addition, spindle length and chromosome segregation are abnormal in Drosophila embryos injected with antibodies against EB1 (Rogers et al., 2002). These results suggest that, during mitosis, EB1 is required to promote MT growth that facilitates interactions with the cell cortex and between MTs of opposite polarity that normally maintain the spindle length.

e. Dis1-TOG. Members of the Dis1-TOG family have also been shown to regulate spindle assembly (reviewed by Ohkura et al., 2001). XMAP215 is required for efficient spindle assembly in mitotic Xenopus egg extracts, since immunodepletion either abolishes spindle formation or results in the assembly of small spindles with very short MTs (Tournebize et al., 2000). More recently, XMAP215 was proposed to have a role in MT nucleation and anchoring at the centrosome (Popov et al., 2002). Also, the related protein in S. pombe Alp14/Mtc1 was shown to be required for bipolar spindle formation, maintenance of spindle length, and centromere movement during the metaphase-anaphase transition (Nabeshima et al., 1998; Garcia et al., 2001). Analyses of a mutation in the Drosophila homologue gene, msps, also revealed the formation of disorganized and short spindles (Cullen et al., 1999; Cullen and Ohkura, 2001), while similar phenotypes have been observed 
in human somatic cells depleted of ch-TOG (Gergely et al., 2003). Ch-TOG is thought to play an essential role in the organization of spindle poles, having a direct influence on the overall organization of the mitotic spindle.

f. TACC. The localization of Dis1-TOG proteins to the spindle poles has been shown to depend on the transforming a acidic coiled-coil (TACC) family of MAPs that, in humans, have been implicated in cancer (for reviews see Gergely, 2002; Raff, 2002). In Drosophila and C. elegans, spindle pole localization of Msps/Zyg-9 requires D-TACC and TAC-1, respectively (Bellanger and Gonczy, 2003; Cullen and Ohkura, 2001; Le Bot et al., 2003; Lee et al., 2001a; Srayko et al., 2003). Moreover, the centrosomal localization of the D-TACC/Msps complex seems to be regulated by Aurora A phosphorylation (Giet et al., 2002). In human cells, TACCs localize exclusive to centrosomes (Gergely et al., 2000a; Gergely et al., 2000b), where they appear to increase the number and/or stability of centrosome-associated MTs (Gergely et al., 2000a). Therefore, together with Dis1-TOG proteins, TACCs probably have an essential role in the organization of the spindle poles and a minor role in stabilization of spindle MTs.

\section{B. Spindle orientation and positioning}

Proper positioning of the mitotic spindle inside the cell is essential for establishment of the cleavage plane. This is of special importance in maintenance of the organization of cells within a developing organism and for cell fate determination resulting from asymmetric cell division (reviewed by Knoblich, 2001). In budding yeast, the spindle must be oriented correctly with respect to the mother-bud axis, since the cleavage plane is predetermined by the bud neck (Yeh et al., 1995). Spindle orientation in animal cells and in yeast requires the interaction of astral MTs with the cell cortex, a process that depends on plus-end MAPs that regulate MT dynamics. Spindle positioning also involves motor proteins as well as a group of proteins that control and establish cell polarity (for reviews see Stearns, 1997; Kaltschmidt and Brand, 2002; Ahringer, 2003; Morris, 2003).

In budding yeast, positioning of the spindle results from a MT-based search mechanism that requires highly dynamic MTs for nuclear migration into the bud (Schuyler and Pellman, 2001b; Shaw et al., 1997). Prior to nuclear migration, MTs search the cell cortex until they interact with Kar9p, a protein that is anchored to the bud cell cortex by myosin, actin, Bud6p, and Bni1p (Gabriel, 1998; Theesfeld et al., 1999; Beach et al., 2000; Kopecka and Miller et al., 2000; Yeh et al., 2000; Yin et al., 2000). Interaction of Kar9p with MTs requires Bim1p, the yeast homologue of EB1 (Miller et al., 2000). This protein is thought to couple MT shortening to nuclear migration (Beach et al., 2000). Kar9p is then loaded onto the bud 
spindle pole, whence it is translocated to cytoplasmic MTs (Liakopoulos et al., 2003). Although homologues of Kar9p have not been found in higher eucaryotes, APC may perform a similar function in these organisms (Bloom, 2000; Liakopoulos et al., 2003). In Drosophila embryos, adherent junctions of neuroepithelial cells are enriched in E-APC and dEB1, which are both required to prevent the intrinsic asymmetric division of these cells, and thus promote symmetric division along the planar axis (Lu et al., 2001). Also, APC2 and its binding partner Armadillo are localized to the sites of cortical spindle attachment, and both participate in the anchoring of spindle MTs during the syncytial nuclear divisions in early Drosophila embryos (McCartney et al., 2001). Furthermore, depletion of EB1 in Drosophila cells results in abnormal spindle positioning (Rogers et al., 2002). Interestingly, Kar9p interacts physically with Stu2p, a member of the Dis1-TOG family (Miller et al., 2000). In this complex, Stu2 is likely to play a role in regulating the dynamics of cytoplasmatic MTs (Kosco et al., 2001). Together, all these results indicate that spindle positioning within cells depends on MAPs that localize to MT plus-ends, either causing local changes in MT dynamic behavior or regulating the interaction of MT plus-ends with cortical components.

The role of motor proteins in spindle orientation has been extensively investigated in budding yeast. It has been demonstrated that spindle positioning requires the function and localization of the dynein/dynactin complex (Li et al., 1993; Muhua et al., 1994; Carminati and Stearns, 1997; Miller et al., 1998; Yeh et al., 2000), and the Kar3p, Kip3p, and Kar2p motor proteins (Cottingham and Hoyt, 1997; DeZwaan et al., 1997). In concert, these motor proteins direct spindle elongation to distal points in the mother cell and the bud. Interestingly, these motor proteins not only affect overall spindle positioning, but they additionally affect MT dynamics, with Kip2p having a stabilizing effect, whereas dynein, Kip3p, and Kar3 promote destabilization (Cottingham and Hoyt, 1997; Miller et al., 1998; Cottingham et al., 1999). Surprisingly, dynein, a minus end- directed motor, is found accumulated at the plus ends of astral MTs. This is thought to result from a direct interaction between dynein and the plus-end-tracking proteins Bik1p and Pac1, the yeast homologues of CLIP-170 and LIS1 respectively (Sheeman et al., 2003). This accumulation at the plus ends of astral MTs may allow anchoring of dynein at specific cortical sites and thereby direct spindle positioning. Although studies in mammalian cells have not progressed as far as in yeast, dynein is also known to be required for spindle orientation and position in higher eukaryotic cells (McGrail and Hays, 1997; O'Connell and Wang, 2000; reviewed by Dujardin and Vallee, 2002).

\section{Microtubule-kinetochore attachment and metaphase chromosome alignment}


Spindle MTs interact directly with chromosomes, and are required to mediate chromosome movements at various stages of mitosis. However, tubulin, the major constituent of MTs, while unable to bind DNA directly in vitro, can do so in the presence of MAPs. Because of these observations, it was originally proposed that the binding of MTs to chromosomes might take place through MAPs that work as linkers (Corces et al., 1978; Wiche et al., 1978). In support of this view, several MAPs had been localized to the kinetochore (De Mey, 1984; Kellogg et al., 1989), and ultrastructural analysis has shown that most kinetochore MTs end up at the outer plate (Ris and Witt, 1981), while only a few terminate in the inner plate or even contact the chromatin (Comings and Okada, 1971). These observations suggest that proteins from the outer kinetochore assemble onto DNA to create an ideal interface for MTs and chromosomes; a model was proposed in which the kinetochore is constructed from functional subunits that are organized around the repeating units of repetitive centromeric DNA (Zinkowski et al., 1991). Accordingly, DNA could only be found associated with the inner plate of the kinetochore (Cooke et al., 1993). Thus, the outer kinetochore plate, together with the ill-defined fibrous corona, may be exclusively composed of proteinaceous material containing MAPs. Initial attempts to isolate MT-kinetochore protein complexes were based on the use of CREST sera from patients with scleroderma autoimmune disease (Balczon and Brinkley, 1987). This study elegantly described the isolation of kinetochore proteins that co-purified with tubulin by affinity chromatography, after chemical crosslinking of tubulin with kinetochores. It was then clear that some structural kinetochore components account for normal MT-kinetochore interactions (Simerly et al., 1990; Bernat et al., 1990), and account for the proper assembly of a functional kinetochore. However, the analysis was limited to proteins recognized by CREST sera, leaving unaddressed many other components that only now are being identified.

\section{A. Chromosome capture and poleward movement}

Pioneer studies in diatoms suggested that the kinetochores usually associate laterally with MTs that have one end attached to the spindle pole (Tippit et al., 1980). Thus, the k-fiber appears to result from an initial lateral contact of the kinetochore with MTs growing from the poles. Ten years later, the same conclusions were drawn for vertebrate cells, in which it was conclusively shown that the predominant mechanism for chromosome attachment results from an interaction between astral MTs and the kinetochore (Hayden et al., 1990; Mitchison, 1990; Rieder, 1990). Therefore, k-fibers are thought to originate from those astral MTs that come into contact with the kinetochore. In the meantime, several observations have suggested that chromosomes of vertebrate cells initially attach laterally to MTs before interacting 
directly with the plus ends (Mitchison and Kirschner, 1985; Huitorel and Kirschner, 1988). This hypothesis was then confirmed by live cell correlative light and electron microscopy studies of ultra-thin serial sections of early prometaphase cells (Rieder and Alexander, 1990; Merdes and De Mey, 1990). Together, these studies revealed that the attachment and subsequent poleward movement of a chromosome correlates with the lateral association of at least a single long MT with one of the kinetochores of the chromosome. Once this association is established, the kinetochore is rapidly transported poleward along the lateral surface of the MT by a mechanism that is not dependent on MT depolymerization. Furthermore, the leading kinetochore does not need to be directly oriented toward the centrosome for this movement to occur (Rieder and Alexander, 1990). These observations led to the proposal that motor proteins located in the fibrous corona of the kinetochore or on the surface of the astral MTs, or both, could be responsible for this particular movement (reviewed by Sluder, 1990). An obvious candidate to drive kinetochore-to-pole movement upon MT attachment is cytoplasmic dynein, a known component of the fibrous corona with minus-end coupled motility (Wordeman et al., 1991; Banks and Heald, 2001). Localization of dynein at the kinetochores is maximal during prometaphase and decreases substantially after kinetochores begin to attach to MTs (King et al., 2000; Wojcik et al., 2001). Overexpression of the 50KDa subunit of dynactin, p50-dynamitin, or microinjection of antibodies against dynein revealed a role in poleward chromosome movement throughout mitosis (Vaisberg et al., 1993; Echeverri et al., 1996; Sharp et al., 2000b). Further, Zw10 and Rough deal (Rod) (Williams et al., 1996; Basto et al., 2000; Chan et al., 2000), both shown to be involved in targeting the dynein complex to kinetochores (Starr et al., 1998), are required for poleward chromosome motion in Drosophila (Starr et al., 1998; Savoian et al., 2000; Wojcik et al., 2001).

\section{B. Chromosome congression}

\section{Microtubule kinetochore interactions}

As the chromosome moves poleward after initial lateral attachment to a single MT, the kinetochore captures the plus ends of neighboring astral MTs, and chromosome motion slows down (Biggins and Walczak, 2003). Studies in vitro have shown that this tethering of MT plus-ends can be explained, in part, by the preferential binding of kinetochores to GTP- rather than GDP-MTs, and to the plus ends preferentially over the MT lattice (Severin et al., 1997). The outer kinetochore plate ultimately becomes saturated with astral MT plus-ends, and consequently, neighboring MTs may bundle with kinetochore MTs to form the stabilized kfiber. At this stage, chromosomes are attached by a single kinetochore to a single pole, i.e., 
they are mono-oriented. From then on, chromosomes oscillate toward and away from that pole, indicating that they are subject to both pulling and pushing forces (Bajer, 1982; Rieder et al., 1986). The process of arranging chromosomes equidistantly from the two poles at metaphase is known as congression (Rieder and Salmon, 1994). A single kinetochore of a bioriented chromosome possesses the capability of congressing to the spindle equator (Khodjakov et al., 1997), indicating that congression can be initiated as soon as a single kinetochore of a chromosome becomes attached to the spindle. When undergoing poleward motion, the centromere is typically stretched, whereas during motion away from the pole it becomes flattened or indented, without exerting a significant pushing force on the chromosome (Skibbens et al., 1993; Khodjakov and Rieder, 1996). For bi-oriented chromosomes, the centromere becomes maximally stretched when both sister kinetochores are moving poleward, and maximally compressed when both are moving toward the spindle equator. This characteristic tendency of a kinetochore to autonomously and abruptly switch between these phases was termed directional instability (Skibbens et al., 1993).

\section{The role of kinesin-like proteins}

It has been demonstrated that during prometaphase, the assembly of spindle-polar MT arrays results in a force that pushes chromosome arms away from the spindle poles (Rieder et al., 1986; Leslie, 1992; Cassimeris et al., 1994; for reviews see Rieder and Salmon, 1994; Ault and Rieder, 1994; Inoue and Salmon, 1995). These "polar ejection forces" or "polar winds" are thought to be involved in chromosome congression, ultimately leading to the formation of the metaphase plate (for reviews see Bajer and Mole-Bajer, 1972; Salmon, 1988; Salmon, 1989; Rieder and Salmon, 1994).

In addition to the physical pushing by MT plus ends, motor proteins bound to chromosome arms can also contribute to the polar ejection force through interaction with astral MTs (Yajima et al., 2003). In this context, several kinesin-related motor proteins have been found to be associated with the chromosome arms: nod in Drosophila (Afshar et al., 1995b), Xklp1 in Xenopus oocytes (Vernos et al., 1995), and chromokinesin in vertebrates (Wang and Adler, 1995; Tokai et al., 1996; Antonio et al., 2000; Funabiki and Murray, 2000). However, recent findings suggested that chromokinesin by itself is only required for orientation of chromosome arms away from the spindle poles and for chromosome oscillation, but not for congression (Levesque and Compton, 2001). A role for chromokinesin in chromosome congression may only be essential in coordination with the spindle-pole organizer protein NuMA (Levesque et al., 2003), suggesting a strong linkage between chromosome positioning and anchoring at the spindle pole. 
Nevertheless, in cells that do not have canonical centrosomes, and therefore lack astral MTs that could produce polar ejection forces, chromosomes still congress. This shows very clearly that our current understanding of the mechanisms of chromosome congression is still far from complete. Indeed, it is very likely that other motor proteins with plus end-directed motility localized at the kinetochore also contribute to chromosome congression during prometaphase. The best characterized example of this class of proteins is CENP-E, originally identified in a screening for novel components of the centromere/kinetochore (Yen et al., 1991; Yen et al., 1992). Ultra-structural and immuno-localization studies showed that during mitosis, CENP-E concentrates in the fibrous corona and outer plate of the mammalian kinetochore from prometaphase through anaphase, and in some MT plus-ends (Cooke et al., 1997; Yao et al., 1997). However, the functional significance of the binding of CENP-E to MT plus ends is not yet clear. CENP-E is one of the largest members (312 KDa) of the kinesin superfamily (Yen et al., 1992; reviewed by Vale and Fletterick, 1997). Surprisingly, a CENP-E-associated minus-end directed motor activity was purified from HeLa cells (Thrower et al., 1995). However, expression of a recombinant fragment of CENP-E was shown to have a plus end-directed motor activity (Wood et al., 1997) making this a controversial issue. One interpretation is that the minus end-directed motor activity arises from another motor protein associated with CENP-E. Indeed, CENP-E was shown to be transported to the poles by dynein upon MT attachment at the kinetochore (Howell et al., 2001). Alternatively, CENP-E could have both plus- and minus-end-directed motor activities, depending on its associations with particular regulatory factors (reviewed by (Grancell and Sorger, 1998). Based on in vitro motility assays, CENP-E was initially proposed to act as a single molecule responsible for attachment of kinetochores to shortening MTs (Lombillo et al., 1995a; Lombillo et al., 1995b; for reviews see Hyman, 1995). However, the fact that disruption of CENP-E function in vivo does not affect kinetochore motion or velocity toward and away from the pole, or even the capacity of most chromosomes to align at the metaphase plate (Schaar et al., 1997; Putkey et al., 2002), raises questions about the significance of the in vitro results (Rieder and Salmon, 1998). Nevertheless, some chromosomes lacking CENP$\mathrm{E}$ at their kinetochores show defects in alignment and in their ability to establish bipolar orientation (Schaar et al., 1997; Wood et al., 1997; Putkey et al., 2002). Therefore, it is reasonable to assume that in the absence of CENP-E, the initial attachment of a single kinetochore, as well as its ability to hold on to shortening MT ends, is not compromised in vivo. However, most CENP-E depleted chromosomes that are able to move to the metaphase plate appear to have their kinetochores bound to only half the normal number of MTs (Putkey 
et al., 2002), suggesting that CENP-E, although not essential, may contribute to efficient MT capture at kinetochores.

Another protein thought to play an indirect role in kinetochore-MT attachment is MCAK/XKCM1, a kinesin-like protein that localizes to centromeres (Desai and Mitchison, 1995; Walczak et al., 1996; Wordeman and Mitchison, 1995). It was recently shown that the function of XKCM1 at the centromeres is required for correct chromosome positioning at the metaphase plate (Walczak et al., 2002). Together with its binding partner, ICIS, MCAK may be part of a mechanism that prevents kinetochore-MT attachment errors, inducing MTs to depolymerize when they are incorrectly attached (Ohi et al., 2003). Indeed, this hypothesis found strong support from a recent study in which it was convincingly shown that chromosome congression defects observed after MCAK depletion from the centromere are due to improper kinetochore attachments (Kline-Smith et al., 2003).

During metaphase, the spindle achieves a steady state, in which it can maintain a defined shape and structure for an extended period. Nevertheless, in vertebrates, chromosomes continue to oscillate along the spindle axis during this time (Bajer, 1982; Roos, 1976). It seems possible that this oscillation process results from MT polymerization at the elongating k-fiber, a process which is balanced by depolymerization of the shortening fiber. This directional instability of kinetochores observed during prometaphase and metaphase may be governed by the activity of antagonistic motors. In this context, it was shown that MTs spontaneously oscillate on surfaces coated with both dynein and kinesin, which have antagonistic MT-based motilities (Vale et al., 1992).

\section{Non-motor MAPs at the kinetochore}

a. CLIP170. Inoué and Salmon (1995) suggested that MT-kinetochore attachment does not require active motor proteins. The idea was that alternative proteins provide the site of attachment and stabilization at the MT-kinetochore interface, while motor proteins move chromosomes along MTs in close association with their dynamic behavior. In this regard, one interesting observation came from localization studies of CLIP-170 during mitosis. This protein localizes to prometaphase kinetochores, but localization is significantly less pronounced in metaphase chromosomes (Dujardin et al., 1998). In addition, Bik1, the budding yeast homologue of CLIP-170, has recently been shown to be required for proper MT-kinetochore attachment of polyploid but not haploid yeast (Lin et al., 2001). Further, extensive analyses of MT-kinetochore attachment in budding yeast (He et al., 2001; Van Hooser and Heald, 2001) clearly pointed to a critical role for non-motor MAPs in this process. Among the MAPs studied, Bik1 was shown to play a central role in kinetochore-MT 
attachment. Finally, in mammals, CLIP-170 interacts with dynein/dynactin at the kinetochores, a process mediated by LIS1 (Coquelle et al., 2002; Tai et al., 2002). Thus, CLIP-170 may be one of early non-motor proteins required for MT-kinetochore interactions, and others are likely to be recognized over time.

b. Dis1-TOG. Stu2, the S. cerevisiae member of the Dis1-TOG family of MAPs, also plays a role in MT-kinetochore attachment (He et al., 2001). A direct role in the stabilization of MT-kinetochore interactions had already been proposed for the S. pombe homologue Dis1 (Nakaseko et al., 2001). Indeed, both Dis1 and Stu2p associate transiently with kinetochores during mitosis. However, only Dis1 appears to bind kinetochores independently of MTs. Likewise, the failure of sister chromatid separation observed in dis1 and STU2 mutant cells has been associated with defects in formation/stabilization of kinetochore MTs.

Fission yeast Alp14 is closely related to Dis1, and it also associates with kinetochores. However, unlike Dis1, Alp14 seems to be required not only for overall MT assembly but also for the function of the kinetochore-attachment checkpoint (Garcia et al., 2001; see next section). Recently, it was found that depletion of the human homologue ch-TOG and its binding partner TACC3 by RNAi interfered with chromosome congression (Gergely et al., 2003). However, there is still no evidence for localization of these proteins to kinetochores. Maybe, like NUMA, these are components of a matrix at the spindle poles that enhances the ability of chromosomes to congress to the metaphase plate (Cullen and Ohkura, 2001; Lee et al., 2001a).

c. CLASPs. Members of the CLASP family share, at the N-terminus, limited sequence conservation with proteins from the Dis1-TOG family (Lemos et al., 2000). While their functional relationship, if any, is still not known, both MAST/Orbit and one of its human homologues, CLASP1, are essential for chromosome congression (Maiato et al., 2002; Maiato et al., 2003a; Maiato et al., 2003b). Moreover, these proteins seem not to be necessary for MT-kinetochore attachment, but rather they appear to regulate MT dynamics at the kinetochore. The available data are fully consistent with a model in which MAST/CLASP1 accumulate at the outer kinetochore corona and maintain attachment to the MTs plus-ends, while allowing the incorporation of new tubulin subunits. Further, dynamic behavior analysis of attached MTs in the absence of CLASP1 suggests that this protein promotes MT rescue and modulates the coordinated dynamic behavior of kinetochore MTs. Supporting these observations, CLIPs, the binding partners of CLASPs, were shown to be anti-catastrophe factors in vivo, as previously shown in fission yeast (Brunner and Nurse, 2000; Komarova et al., 2002). 
d. APC and EB1. Like other +TIPs, there is some evidence that APC and EB1 localize to the kinetochores, albeit in a MT-dependent manner (Fodde et al., 2001; Kaplan et al., 2001; Tirnauer et al., 2002a). Consequently, a role for these proteins in chromosome segregation has been proposed. Binding of EB1 to MT plus-ends at the kinetochore interface is restricted to polymerizing MTs, suggesting that the protein modulate MT's dynamic behavior (Tirnauer et al., 2002a). Deletion of Bim1 and Mal3 in yeast results in hypersensitivity to MTdestabilizing agents, aberrant spindle behavior, and chromosome mis-segregation (Beinhauer et al., 1997; Schwartz et al., 1997). Depletion of EB1 from Drosophila cells in culture showed that EB1 influences MT dynamics during interphase, with possible consequences for spindle assembly during mitosis (Rogers et al., 2002).

e. Chromosomal passengers. Another group of proteins that may play a role in chromosome congression are the chromosomal passengers. Expression of dominant-negative mutants of INCENP disrupted localization of endogenous INCENP and induced defects in chromosome congression (Eckley et al., 1997; Mackay et al., 1998). More recently, it was found that disruption of any member of this complex by any of various approaches compromises metaphase chromosome alignment (Adams et al., 2001; Kaitna et al., 2002; Kallio et al., 2002; Murata-Hori and Wang, 2002; Carvalho et al., 2003; Ditchfield et al., 2003; Hauf et al., 2003; Honda et al., 2003). Further, the passenger complex is required for a particular aspect of MT-kinetochore interaction, namely, chromosome bi-orientation (Adams et al., 2001; Tanaka et al., 2002; Murata-Hori and Wang, 2002; Hauf et al., 2003; reviewd by Stern, 2002). The mechanism of action of Aurora B kinase to ensure proper chromosome orientation and consequent alignment at the metaphase plate can partly be explained by this protein's regulatory role in the formation of the passenger protein complex. However, this function may only be an indirect consequence of disruption of the centromere structure, resulting in compromised kinetochore function. Accordingly, it was recently shown that Aurora B regulates the normal targeting of motor proteins like dynein and CENP-E to kinetochores (Murata-Hori and Wang, 2002). In addition, the role of the chromosomal passenger complex in chromosome bi-orientation may be partly explained by a functional interaction with MCAK and ICIS, which were proposed to prevent kinetochore-MT attachment errors (Ohi et al., 2003). In this correction mechanism, Aurora B plays a central role in the phosphorylation-dependent regulation of centromeric MCAK (Andrews et al., 2004).

\section{Kinetochore-attachment checkpoint}

\section{Essentials of checkpoint function}


During prometaphase, when the initial interaction between spindle MTs and kinetochores takes place, it is crucial that cells do not initiate anaphase until all chromosomes are properly bi-oriented. Zirkle (1970) was the first to propose that a signal emanating from the chromosomes or the spindle was able to arrest cells at prometaphase until all kinetochores became attached to MTs. Subsequently, the role of mis-oriented chromosomes in the generation of a diffusible inhibitor of anaphase onset was demonstrated (Sluder et al., 1994), and direct evidence for the existence of a checkpoint that monitors kinetochore attachment to the spindle was obtained (Rieder et al., 1994). These results were also consistent with previous observations suggesting that tension exerted on the chromosomes is monitored by a checkpoint (Nicklas and Koch, 1969). Later, it was shown that in the absence of tension produced, kinetochores transmit an inhibitory signal that delays the metaphase-anaphase transition (Li and Nicklas, 1995; for reviews see Murray, 1995; Nicklas, 1997). Also in cultured vertebrate cells, it was found that molecules in or near the unattached kinetochore of a mono-oriented chromosome are able to inhibit the metaphase-anaphase transition (Rieder et al., 1995). The first biochemical evidence for a link between kinetochore attachment and checkpoint function was provided by the phosphoepitope-specific monoclonal antibody 3F3/2 (Gorbsky and Ricketts, 1993). This antibody preferentially stains kinetochores that are not under tension, and microinjection of 3F3/2 antibody into cells delays the metaphaseanaphase transition without affecting chromosome movement (Campbell and Gorbsky, 1995). Moreover, it was shown that tension alters the phosphorylation of kinetochore proteins, as determined by 3F3/2 staining, suggesting that kinetochore protein dephosphorylation caused by tension constitutes the signal for anaphase onset ( $\mathrm{Li}$ and Nicklas, 1997; Nicklas et al., 1998; Nicklas et al., 1995). Whether the signal is tension per se or MT occupancy, which increases after tension is applied (King and Nicklas, 2000), is still the subject of much controversy and requires definitive resolution.

Cell fusion experiments indicated that unattached kinetochores on one spindle did not block anaphase onset in a neighboring mature metaphase spindle that lacked unattached kinetochores in a common cytoplasm (Rieder et al., 1997). These findings demonstrated that in vertebrate cells, the inhibitory activity associated with an unattached kinetochore is not freely diffusible, but instead acts locally within the spindle containing the unattached kinetochore. Furthermore, these experiments revealed that once a mature spindle entered anaphase, the neighboring spindle immediately followed, entering anaphase regardless of the presence of mono-oriented chromosomes. Thus, anaphase onset in the mature spindle triggers 
anaphase in the adjacent spindle, overriding the inhibitory signal produced by unattached kinetochores.

Based on the observation that the spindle checkpoint is continuously active in cells treated with drugs that disrupt spindle MTs (for reviews see Rieder and Palazzo, 1992; Jordan and Wilson, 1998), a genetic screen was carried out in yeast to isolate mutations that rendered cells insensitive to spindle damage. As a result of these screens, the first components of the kinetochore-attachment checkpoint (also incorrectly called by many the spindle-assembly checkpoint) were identified (Hoyt et al., 1991; Li and Murray, 1991; for reviews see Murray, 1992; Murray, 1995). These studies identified six genes, BUB1-BUB3 (for "budding uninhibited by benzimidazole") and MAD1-MAD3 (for “mitotic arrest-deficient”), whose products are required for cell-cycle arrest in response to MT poisons that induce depolymerization. Our knowledge of the function of these proteins has advanced significantly, and new members of the checkpoint have also been discovered (for reviews see Rudner and Murray, 1996; Skibbens and Hieter, 1998; Amon, 1999). These include Mps1, a protein kinase that is required for both normal spindle pole body duplication (Winey et al., 1991) and for delaying mitotic exit in yeast having SPB defects, or in the presence of nocodazole (Weiss and Winey, 1996). Another is Cdc55 (Minshull et al., 1996; Wang and Burke, 1997), a regulatory subunit of protein phosphatase 2A (PP2A) that has been shown to be involved in regulating exit from mitosis (Healy et al., 1991; Gomes et al., 1993).

Animals, however, do not have a Mad3 homologue, although a Bub1-Related kinase, BubR1, has been identified. BubR1, like Bub1, has a Mad3 N-terminal domain containing Cdc20- and Bub3-binding sites, and a C-terminal protein kinase domain (Jablonski et al., 1998; Taylor et al., 1998). BubR1 was found to be an interactor with CENP-E at the kinetochores (Chan et al., 1998), and was recently reported to be required for chromosome congression, which is possibly mediated by this particular interaction (Ditchfield et al., 2003).

Components of the kinetochore-attachment checkpoint are widely conserved among organisms, and homologues of genes involved in checkpoint response have been found in many eukaryotes (Abrieu et al., 2001; Basu et al., 1999; Basu et al., 1998; Cahill et al., 1998; Chen et al., 1996; Dobles et al., 2000; Fisk and Winey, 2001; Jin et al., 1998; Kitagawa and Rose, 1999; Li and Benezra, 1996; Martinez-Exposito et al., 1999; Michel et al., 2001; SharpBaker and Chen, 2001; Stucke et al., 2002; Taylor et al., 1998; Taylor and McKeon, 1997).

Mad2 was one of the first checkpoint proteins to attract particular interest, due to its highly dynamic distribution during early stages of mitosis. Mad2 localizes to unattached kinetochores in prometaphase and disappears from kinetochores at metaphase, suggesting a 
role in the inactivation of the checkpoint (Chen et al., 1996; Li and Benezra, 1996; Shah and Cleveland, 2000). In contrast to 3F3/2 phosphoepitopes that are though to be present on kinetochores in the absence of tension, loss of tension is insufficient to induce Mad2 accumulation on kinetochores, whereas unattached kinetochores consistently showed the presence of kinetochore-associated Mad2 (Waters et al., 1998). This distinct behavior of Mad2 seems to be regulated by phosphorylation of kinetochore proteins independently of Bub1 kinase activity (Sharp-Baker and Chen, 2001; Waters et al., 1999). Thus, a kinetochoreMT attachment mechanism that is sensitive to kinetochore phosphorylation inhibits Mad2 accumulation at kinetochores and should allow anaphase onset. Accordingly, microinjection of antibodies to Mad2 into mammalian cells induces premature anaphase (Gorbsky et al., 1998).

Like Mad2, Bub1, BubR1, and Bub3 also localize to unattached kinetochores and were hypothesized to help monitor and/or generate the inhibitory signal that delays the onset of anaphase (Taylor and McKeon, 1997; Taylor et al., 1998; Jablonski et al., 1998; MartinezExposito et al., 1999). However, while Mad2 seems to be monitoring spindle attachment to the kinetochores, other proteins like Bub1, BubR1, and Bub3 seem to monitor tension at the kinetochores (Taylor et al., 1998; Skoufias et al., 2001; Tang et al., 2001; for reviews see Gillett and Sorger, 2001; Hoyt, 2001). This apparent branching of the kinetochore-attachment checkpoint has been refuted by work from other labs (Hoffman et al., 2001; Shannon et al., 2002). However, recent evidence from Drosophila lysed cells has shown that distinct checkpoint proteins do appear to behave differently with respect to MT attachment and tension (Logarinho et al., 2004). It was demonstrated that while strong Mad2 and Bub1 kinetochore accumulation only occurs in the absence of MTs, BubR1 and Bub3 fail to accumulate at the kinetochore only when 3F3/2 phosphoepitopes are fully dephosphorylated, which are indicators of tension across the kinetochores. While this provides strong evidence for a branching of the checkpoint monitoring two distinct events, ambiguity remains - due to the different model systems studied - which components of the kinetochore-attachment checkpoint belong to which branch.

BubR1 has been shown to interact directly with the kinesin-like motor CENP-E, now thought to report the status of kinetochore attachment to the checkpoint (Abrieu et al., 2000; Chan et al., 1998). More recently, a biochemical approach using purified components and Xenopus egg extracts yielded results suggesting that CENP-E is an activator of BubR1, possibly responsible for its kinase activity and therefore checkpoint silencing (Mao et al., 
2003). This interaction may be part of a “MT counting”-mechanism that responds to MT attachment and tension induced at the kinetochores.

\section{Coupling of microtubule-kinetochore attachment to bi-orientation and checkpoint} control

How does MT attachment couple to chromosome bi-orientation and to inactivation of the kinetochore-attachment checkpoint? The answer seems to involve a role for the chromosomal passenger Aurora B. In addition to motor proteins, Aurora B is required for normal targeting of BubR1 and MAD2 to kinetochores (Murata-Hori and Wang, 2002; Ditchfield et al., 2003; reviewed by Shannon and Salmon, 2002). Moreover, the budding yeast homologue, Ipl1, was initially shown to be required for the mitotic delay by chromosomes whose kinetochores are not under tension (or are not fully occupied with MTs), but it is not required for arrest induced by spindle depolymerization, indicating that Ipl1 is required to monitor tension at the kinetochore (Biggins and Murray, 2001). In contrast, overexpression of dominant-negative Aurora B, or microinjection of Aurora B antibodies in vertebrate cells, caused an overriding of the spindle checkpoint, bypassing spindle checkpoint activation in response to either spindle depolymerization or a lack of tension caused by the action of MT-stabilizing drugs (Kallio et al., 2002; Murata-Hori and Wang, 2002). Further, inhibition of Aurora B by selective drug targeting, in vertebrate cells also overrides the checkpoint when cells are arrested by taxol or monastrol (Ditchfield et al., 2003; Hauf et al., 2003). However, cells in nocodazole remained arrested for longer periods than cells in taxol (although never as long as do control cells). These observations suggest that Aurora B function is required for normal checkpoint function, whether activated by loss of tension or by reduced MT occupancy at the kinetochores. Furthermore, Aurora B is also required for checkpoint response induced by MT depolymerization after prolonged mitotic arrest. Indeed, the single Aurora kinase from fission yeast, and its partner, Survivin, are likely to be required for the MT-attachment response by the checkpoint (Petersen and Hagan, 2003). However, in vertebrate cells, Survivin seems only to be required for effective checkpoint activation when tension at the kinetochores is compromised by the presence of taxol (Carvalho et al., 2003; Lens et al., 2003). This pattern can be explained by the requirement of Survivin to stably maintain BubR1 at the kinetochores.

\section{Microtubule plus-end-tracking proteins, kinetochore attachment, and checkpoint}

\section{response}

In addition to a role in the targeting of motor and checkpoint proteins to the spindle, Ipl1 was recently shown to regulate the function of multiple kinetochore proteins by 
phosphorylation (Cheeseman et al., 2002). Among these are members of the multisubunit Dam1/Duo complex (Cheeseman et al., 2001; Kang et al., 2001; Janke et al., 2002; Li et al., 2002), which are kinetochore- and spindle-associated proteins involved in MT-kinetochore attachment (Hofmann et al., 1998; Cheeseman et al., 2001; Enquist-Newman et al., 2001; Jones et al., 2001; Janke et al., 2002).

It is reasonable to posit an eventual functional interaction between CLASPs, chromosomal passengers, and the Dam1/Duo complex at the kinetochore. Dam1 shows genetic interactions with Stu1, the single orthologue of CLASPs in S. cerevisiae, (Jones et al., 1999) and it was recently demonstrated to associate directly with Ipl1 and Sli15, the yeast orthologues of the Aurora-INCENP complex, respectively (Kang et al., 2001). Moreover, Ipl1 phosphorylates Dam1 both in vitro and in vivo and, together with sli15, these two proteins were shown to be required for chromosome segregation. Furthermore, dam1 mutants carry out anaphase-like events with high levels of Clb2p (the cyclin B orthologue in yeast) and without checkpoint inactivation; this behavior was also observed after RNAi of MAST in Drosophila tissue-culture cells (H. Maiato, unpublished observations). Furthermore, interference of MAST function either by mutations or by RNAi in Drosophila tissue culture cells leads to the formation of single nucleated highly polyploid cells (Lemos et al., 2000; Maiato et al., 2002), indicative of a checkpoint override and consequent cycles of DNA reduplication without chromosome segregation.

\section{Microtubule motors, and inactivation of the spindle checkpoint}

Among the checkpoint proteins analyzed thus far, Mad2 shows the most significant reduction in its level of accumulation at metaphase kinetochores. This behavior appears to be due to the inability of the kientochore to accumulate more Mad2 as MTs saturate the kinetochore-binding sites, coupled to the rapid movement of Mad2 along k-fibers toward spindle poles (Howell et al., 2000), a translocation mediated by the motor activity of cytoplasmic dynein/dynactin (Howell et al., 2001). This observation led to the proposal that dynein/dynactin act to inactivate the kinetochore-attachment checkpoint by transporting kinetochore proteins, such as Mad2, to the poles. In support of this prediction, Drosophila embryos with mutations in cytoplasmic dynein have been shown to accumulate Zw10 at metaphase kinetochores (Wojcik et al., 2001). Zw10 and Rod are required for the kinetochore-attachment checkpoint in flies and humans (Basto et al., 2000; Chan et al., 2000). These were the first checkpoint components to be identified that do not have obvious homologues in yeast, suggesting that metazoans require an elaborate checkpoint in order to monitor complex kinetochore functions. 


\section{Chromosome segregation}

\section{A. Anaphase A}

Once all of the chromosomes have congressed, sister kinetochores oscillate within a relatively narrow region, and the cell is considered to be at metaphase. At this stage, spindle MTs display one of their most enigmatic properties, known as poleward MT flux: this, in practical terms, resembles the previously observed MT treadmilling at steady-state tubulin concentrations in vitro. Studies in living cells have shown that during metaphase, there is a flux of tubulin that results from tubulin incorporation at the kinetochores in existing MTs and disassembly near the poles (Mitchison, 1989). Poleward MT flux seems to be a general property of spindle MTs, rather than specific to kinetochore MTs, since it can also be observed in spindles assembled in vitro in the absence of chromosomes or centrosomes (Sawin and Mitchison, 1991b; Sawin and Mitchison, 1994). Interestingly, in cells, poleward MT flux also seems to be completely independent of kinetochore MT plus-end dynamics, and is capable of producing pulling forces that generate centromeric tension in bi-oriented chromosomes (Waters et al., 1996), suggesting that this intrinsic MT property plays an active role in chromosome movement and segregation.

During anaphase, sister kinetochores separate and move poleward (Rieder and Salmon, 1994). Poleward movement during anaphase A is coupled to the disassembly of tubulin subunits at the kinetochores, known as the “pac-man” model (Gorbsky et al., 1987; Gorbsky et al., 1988; Koshland et al., 1988; Coue et al., 1991). However, a slow poleward flux of kinetochore MTs continues, indicating that, at this stage, kinetochore MTs also disassemble at their minus ends near the spindle poles (Gorbsky et al., 1987; Gorbsky et al., 1988; Koshland et al., 1988; Coue et al., 1991; Mitchison and Salmon, 1992; Zhai et al., 1995; Waters et al., 1996; Desai et al., 1998; reviewed by Inoue and Salmon, 1995). A recent study on kinetochore-MT dynamics in spindles assembled in vitro in Xenopus egg extracts has provided the best description of spindle MT flux at the kinetochore interface, and the results supported roles for both tubulin disassembly at the kinetochore and poleward flux in the movement of chromosomes during anaphase (Maddox et al., 2003).

In addition to the pulling force derived from intrinsic MT behavior and from disassembly of MTs from the kinetochore, some results suggest that motor proteins play a role in chromosome segregation during anaphase A. For instance, in vitro, the kinesin-like protein CENP-E couples kinetochore attachment to depolymerization of MTs at their plus-ends (Lombillo et al., 1995a). Moreover, although this finding is still controversial, a minus end- 
directed MT motor activity has been associated with CENP-E, which is found at kinetochores also during anaphase (Thrower et al., 1995). Additionally, MCAK, another member of the kinesin superfamily with a prominent role in promoting MT depolymerization (reviewed by Walczak, 2003) is also found at the centromeric region throughout anaphase (Wordeman and Mitchison, 1995). It is hard to imagine a centromeric protein having an active role in chromosome segregation driven by MT depolymerization at kinetochores. Nevertheless, overexpression of a motor-less construct, or antisense silencing of MCAK, has been found to cause chromosome mis-segregation during anaphase (Maney et al., 1998), although this is likely to result from the inability of the cell to correct deficient kinetochore-attachments, as discussed above, or due to an indirect effect in the proper targeting or assembly of other kinetochore proteins, which could compromise kinetochore function. Alternatively, MCAK is also found at the polar regions close to the centrosomes and thus may have an active role in promoting depolymerization at the poles as it was recently suggested (Holmfeldt et al., 2004). This may have strong implications for the mechanism of poleward MT flux. Accordingly, it was recently shown that two members of the KinI MT-destabilizing proteins in Drosophila, KLP59C and KLP10A, mediate anaphase kinetochore- and pole-associated depolymerization, respectively (Rogers et al., 2004). It is relevant, however, that early studies on nucleotide requirements for chromosome movement clearly indicated that only anaphase B-, but not anaphase A-associated motion, requires ATP (Cande, 1982). It will be of great interest to clarify this issue and to test the role of these KinI proteins in a vertebrate system, where the relation between chromosome movements and spindle elongation during anaphase A and B are well understood.

Dynein also moves cargo toward the minus-ends of MTs, but it is either absent or significantly depleted from kinetochores upon onset of anaphase. In addition to motor proteins, ZW10 and Rod were shown to have a role in poleward chromosome movement (Savoian et al., 2000), and thus are good candidates for anaphase mediators.

More recently, CLASP1 was implicated in regulating the dynamics of kinetochoreattached MTs (Maiato et al., 2003a). Interestingly, CLASP1 remains associated with kinetochores through anaphase, suggesting that it may modulate the disassembly of tubulin subunits at the kinetochores during chromosome segregation. However, no direct evidence for this hypothesis has been put forward.

Besides MTs, there is also growing evidence that other components of a spindle matrix are also involved in the generation of chromosome movement during anaphase (see reviews by Pickett-Heaps et al., 1984; Pickett-Heaps et al., 1997; Pickett-Heaps and Forer, 2001; 
Scholey et al., 2001; Wells, 2001; Johansen and Johansen, 2002). These may include cytoplasmic dynein, Eg5, NuMa and others, but further work is necessary here.

\section{B. Anaphase B}

In late anaphase (anaphase B), MTs are no longer disassembled at the kinetochore, but the chromosomes continue to move toward the poles. This is partly due to the poleward flux of kinetochore MTs (Mitchison and Salmon, 1992). During anaphase B, the interpolar distance increases as result of separation of the spindle poles themselves, and also due to the sliding of interdigitated MTs that organize the spindle midzone (Masuda et al., 1988). The spindle poles play an active role, contributing to spindle elongation by a cytoplasmic dynein-mediated interaction between astral MTs and the cell cortex, pulling the poles apart (Aist et al., 1993; Sharp et al., 2000a). Additionally, the spindle midzone contains a variety of motor proteins, including members of the unrelated kinesin proteins CHO1 and CENP-E, which possess MT cross-linking activities and therefore are likely to be involved in this process (Yen et al., 1991; Nislow et al., 1992; Liao et al., 1994). Nevertheless, there is a clear gap in our understanding of mitosis here that demands further investigation on the mechanisms of anaphase B.

\section{Mitotic exit and cytokinesis}

Mitosis is separated into two distinct events: karyokinesis and cytokinesis. Cytokinesis is most often defined as the final stage of mitosis, or the stage at the end of mitosis when division of the cytoplasm between the two daughter cells takes place. However, this definition involves several conceptual difficulties, since it is known that the initial formation of the contractile ring that will divide the cytoplasm begins during anaphase $\mathrm{B}$. The contractile ring is an actin-myosin structure that forms at the cell equator around the MT bundles of the spindle midzone and constricts inward pinching the cell in two (Schroeder, 1972; Schroeder, 1973; Fujiwara et al., 1978; reviewed by Rappaport, 1986). The spindle midzone is composed mainly of anti-parallel interpolar MTs that are formed between separated sister chromatids after anaphase onset (Mastronarde et al., 1993; McIntosh, 1971). However, there is evidence that the MTs that form the spindle midzone can also polymerize de novo (Canman et al., 2000). The nucleation of these newly formed MTs is likely to be mediated by $\gamma$-tubulin, which has also been implicated in the completion of cytokinesis, since it is required for the formation of the midbody matrix (Julian et al., 1993; Shu et al., 1995), an electron-dense structure that results from the compaction of the MTs from the spindle 
midzone at the final stages of cell division, when observed under the EM. Cytokinesis comprises several morphological stages: specification and positioning of the cleavage site; cleavage-furrow formation and constriction; spindle midzone formation; and cell separation or abscission. Here we discuss the role of MTs and MT-associated proteins involved in these processes. For those interested in a detailed description of cytokinesis, this can be found elsewhere (Glotzer, 2001).

\section{A. Coordination between karyokinesis and cytokinesis}

Cytokinesis is intrinsically coupled to the exit from mitosis, and it must not occur before spindle assembly and chromosome segregation. Accordingly, inactivation of Cdk1, a major regulator of mitosis, is required for the initiation of cytokinesis in cultured cells (Wheatley et al., 1997). Other mitotic kinases like Polo and Aurora B have also been implicated in this coordination. For instance, overexpression of Plo1, the Polo orthologue in S. pombe, is able to induce the formation of ectopic septa, structures evolutionarily related to the cleavage furrow in higher eukaryotes (Ohkura et al., 1995). In Drosophila, Polo is required for the correct formation of the spindle midzone during spermatogenesis (Carmena et al., 1998; Herrmann et al., 1998). Moreover, Polo-like kinases (PLKs) in humans and Drosophila were shown to localize to the spindle midzone (Golsteyn et al., 1995; Logarinho and Sunkel, 1998). Whether Polo is directly associated with MTs is not known, but it has preferential MT-associated substrates like $\beta$-tubulin (Tavares et al., 1996) and Asp, a MAP that may correspond to the Drosophila functional homologue of NuMA (do Carmo Avides et al., 2001). Asp was recently shown to play a role in the formation of the spindle midzone and cytokinesis (Riparbelli et al., 2002; Wakefield et al., 2001). In addition, PLKs coimunoprecipitate with and phosphorylate CHO1/MKLP1/Pavarotti/ZEN-4, a kinesin-like protein required for the organization of the spindle midzone during cytokinesis (Lee et al., 1995; Adams et al., 1998; Powers et al., 1998; Raich et al., 1998; Kuriyama et al., 2002). CHO1/MKLP1 was found to interact with f-actin, suggesting that it can link MTs from the spindle midzone with the contractile ring during furrow ingression (Kuriyama et al., 2002). However, CHO1 is only required for the terminal phase of cytokinesis. Interestingly, both Polo and Pavarotti are mutually required for their normal targeting to the central spindle. This suggests that the plus-end directed motility of Pavarotti works to mobilize the regulatory activity of Polo during cytokinesis and, on the other hand, phosphorylation by Polo regulates the motor activity of Pavarotti (Adams et al., 1998; Carmena et al., 1998). 
Another kinesin-like protein whose function during cytokinesis seems to overlap with that of Pavarotti is KLP3A, which was shown to play a role in the formation of the spindle midzone and cleavage furrow (Williams et al., 1995). These kinesin molecules could function in the promotion of MT cross-linking or in MT-dependent transport of components required for the ingression of the furrow and completion of cytokinesis.

PLKs have also been implicated in the activation of Cdk1 during entry into mitosis (Kumagai and Dunphy, 1996; Qian et al., 1998), activation of the anaphase-promoting complex (for reviews see Zachariae and Nasmyth, 1999; Peters, 2002), and Cdk1 inactivation during mitotic exit (Charles et al., 1998; Descombes and Nigg, 1998; Kotani et al., 1998; Shirayama et al., 1998). These observations suggest that one main role of PLKs during late stages of cell division is to couple the down-regulation of Cdk1 activity with the promotion of cytokinesis.

Aurora B kinase, which has also been shown to be essential for cytokinesis (see next section), seems to require the activity of Cdk1 for proper relocation from the centromeres to the spindle midzone (Wheatley et al., 1997; Murata-Hori et al., 2002). A possible model to explain these results is that Aurora B and associated passenger proteins remain at the centromeres until Cdk1 activity is reduced during anaphase onset. Subsequently, passenger proteins transfer to the spindle midzone. This translocation may involve an association with CHO/MKLP1, but this issue remains controversial (Kaitna et al., 2000; Adams et al., 2001; Giet and Glover, 2001).

\section{B. Role of microtubules in the specification and positioning of the cleavage site}

Classical studies of cytokinesis have indicated that two asters from independent spindles within the same cell are able to induce formation of an ectopic cleavage furrow (Rappaport, 1961). Additionally, it was shown that increasing the distance between the two asters in a cell leads to failure of cytokinesis (reviewed by Rappaport, 1986). The immediate consequence of these observations is that the correct MT environment is what determines the localization of the cleavage site. On the other hand, studies in tissue culture cells have led to the hypothesis that the signals determining furrow position arise from the chromosomes, and probably involve the chromosomal passenger proteins (Earnshaw and Bernat, 1991; Wheatley and Wang, 1996; Eckley et al., 1997)

However, a number of findings have questioned whether chromosomes have any active role in late mitotic events. Early studies of cleavage furrow formation in sea urchin eggs and grasshopper spermatocytes that lack chromosomes showed that these cells can progress normally through cytokinesis (Hiramoto, 1971; Zhang and Nicklas, 1996). Moreover, it was 
shown that functional cleavage furrows could form in vertebrate cells between two asters that were not connected by an intervening spindle (Rieder et al., 1997). Together, these results support the hypothesis that bundles of interpolar MTs, rather than chromosomes or spindle MTs, play a major role not only in establishing the site of furrow formation but also in its stabilization (Cao and Wang, 1996; Wheatley and Wang, 1996; Wheatley et al., 1998). Indeed, recent genetic and micromanipulation experiments demonstrated that MTs, regardless of whether or not the spindle is bipolar, are the only structural constituent of the mitotic apparatus required for induction of the cleavage furrow (Sampaio et al., 2001; Alsop and Zhang, 2003; Canman et al., 2003). However, two interacting arrays of astral MTs (deprived of chromosomes) are not able to sustain the normal spindle architecture in vertebrate cells, suggesting that chromosomes are dispensable for spindle morphogenesis per se, yet still have some spindle stabilizing properties (Faruki et al., 2002). Moreover, these observations do not rule out a role for the chromosomal passenger complex in the specification of the cleavage furrow. For instance, INCENP can be found in the ectopic furrows that formed between two independent spindles during mitosis in cultured cells (Savoian et al., 1999). Interestingly, CHO1 was also present in the ectopic furrows, whereas CENP-E was not, strongly arguing against an essential role for CENP-E in the completion of cytokinesis. Furthermore, cells with monopolar spindles that completed cytokinesis show accumulation of INCENP at the cleavage furrow, suggesting that chromosomes provide MT-stabilization factors required for the determination of the cell division plane (Canman et al., 2003). More recently, genetic analysis of Drosophila mutants that produce secondary spermatocytes devoid of chromosomes supported the notion that normal spindle assembly and cytokinesis can still occur in the absence of chromosomes (Bucciarelli et al., 2003). This same study revealed that despite of the chromosome-free spindles, Aurora B kinase still targets to the spindle midzone, suggesting that association of Aurora B (and probably the other passenger proteins) with chromosomes is not a prerequisite for this protein's accumulation or functioning at the central spindle during cytokinesis. Moreover, there is evidence for a diffusible pool of chromosomal passenger proteins in the cytoplasm, which might account for their independent binding to MTs, likely to be mediated by INCENP (Wheatley et al., 2001b; see also comment by Wang, 2001). This could readily explain why chromosomal passengers themselves are required for cytokinesis, while chromosomes are not. Accordingly, functional inactivation of INCENP, Aurora B, and Survivin has confirmed roles of these passenger proteins in cytokinesis (Mackay et al., 1998; Cutts et al., 1999; Fraser, 1999; Li et al., 1999; Adams et al., 2001; Giet and Glover, 2001; Murata-Hori et al., 2002; Carvalho et al., 2003; Honda et al., 2003). 


\section{A. Organization of the spindle midzone and formation of the cleavage furrow}

Is the spindle midzone required for contractile ring closure during cytokinesis? Recent studies in Drosophila revealed a functional cooperation between the spindle midzone and the contractile ring during cytokinesis (Adams et al., 1998; Giansanti et al., 1998; reviewed by Gatti et al., 2000). Moreover, it seems that distinct genes are required for particular aspects of cytokinesis, such as spindle-midzone formation, actin accumulation at the cell equator, contractile ring assembly or disassembly, and membrane behavior (Somma et al., 2002). Thus, it has been proposed that interactions among MTs from the spindle midzone, the actinmyosin contractile ring, and the plasma membrane are all required for normal cytokinesis to take place.

In addition to motor and passenger proteins, other non-motor MAPs have been found associated with the spindle midzone (Jiang et al., 1998; Lemos et al., 2000; Maiato et al., 2003a). Two of these MAPs, PRC1 and CLASP1, share an ability to promote MT bundling (Maiato et al., 2003a; Mollinari et al., 2002). While a role for CLASPs in cytokinesis has not been demonstrated, PRC1 was shown to be essential for the maintenance of the spindle midzone during cytokinesis and is thought to be directly regulated by Cdk1 phosphorylation (Jiang et al., 1998; Mollinari et al., 2002). Also, the orthologue of PRC1 in S. cerevisiae, Ase1, was shown to be necessary for spindle elongation during anaphase $\mathrm{B}$, probably due to its MT cross-linking or cortical binding properties (Pellman et al., 1995; Schuyler et al., 2003). Interestingly, Ase1 was identified in a screen for mutations that show synthetic lethality with a bik1 null mutation: since BIK1 is the counterpart of CLIP-170 in this organism, the suggestion is that the CLIP family is also involved in this process.

\section{B. Splitting cells apart}

Cytokinesis is a completely reversible process until the very last moment of physical separation, or abscission, of the two daughter cells. This ultimate process requires the complete dissolution of the midbody matrix that forms the last intercellular bridge. Initially, the midbody matrix was simply thought of as a remnant of the stem body from the spindle midzone that resulted from the closure of the contractile ring. However, a growing body of evidence supports a more active and regulated role in the transition from mitosis to the G1 phase of the next cell cycle. First, several motor proteins, such as MKLP1/CHO1, XKLP1, CENP-E and dynein, were shown to reside in the midbody matrix, suggesting active roles for them in the mechanics of cell separation (Nislow et al., 1990; Yen et al., 1991; Vernos et al., 1995; Karki et al., 1998). Second, biochemical studies on isolated midbodies from CHO cells originally suggested the existence of several other proteins (including MAPs), in addition to 
tubulin and actin, in this structure (Mullins and McIntosh, 1982). Third, recent studies have identified signaling proteins, including kinases, phosphatases, GTPases or proteases, membrane proteins, tight- junction components, and vesicle-fusion proteins, within the midbody region (reviewed by Zeitlin and Sullivan, 2001). Important regulatory proteins like Polo and Aurora B kinases (and the other passenger proteins), as well as PP1 phosphatase, were shown to localize to this structure (Lee et al., 1995; Logarinho and Sunkel, 1998; Arnaud et al., 1998; Terada et al., 1998; Trinkle-Mulcahy et al., 2003). More recently, a systematic proteomic/RNAi analysis of conserved midbody components has identified 158 proteins shown to be required for cytokinesis. Surprisingly, most of the proteins identified fall in the categories of motor, vesicular trafficking, and secretory proteins, suggesting that the formation of a new membrane surface area requires proteins involved in vesicle transport and fusion. In this context, MTs were proposed to be involved in vesicle accumulation and membrane deposition during furrowing, via a novel MT structure, identifified in Xenopus eggs, called the furrow MT array (FMA) (Danilchik et al., 1998). This structure seems to be required for the addition of new plasma membrane during closure of the cleavage furrow (Danilchik et al., 1998; Field et al., 1999; Straight and Field, 2000).

Several studies have suggested an active role of the centrosomes linking the successful completion of cytokinesis with progression from $\mathrm{G}_{1}$ to the $\mathrm{S}$ phase of the cell cycle. If centrosomes are surgically removed from interphase cells with a micro-needle (Hinchcliffe et al., 2001), or by laser ablation from mitotic cells (Khodjakov and Rieder, 2001), mitotic cells can still assemble spindles, but cytokinesis is seriously compromised. This is likely to be due to the absence of astral MTs, which are required for proper spindle positioning. One immediate implication for cell-cycle progression is that some mechanism must be in place to ensure that dividing animal cells receive the appropriate number of functional centrosomes.

\section{Concluding remarks}

In this review we have attempted to comprehensively organize the mitotic roles of the many types of MAPs (Figure 6 and Table 1). In summary, we can conclude that most MAPs described here are essential for at least one aspect of mitosis, including the regulation of MT dynamics in order to build a functional mitotic spindle, the capture and movement of chromosomes, the correction of improper interactions between MTs and chromosomes, and the closure of the contractile ring during cytokinesis. Some MAPs appear to use MTs only as a structural scaffolding, while others have profound influence on the behavior of MTs. If indeed the intrinsic properties of MTs play a major role in mitosis, such a role would not be 
possible in the absence of the diversity, versatility, and regulatory properties of MAPs. Only now we are starting to understand how these properties, initially observed and tested in vitro, can be translated to in vivo situations in order that they can accomplish one of the most complicated biological problems - the faithful division of a cell.

\section{Acknowedgements}

We would like to thank Conly Rieder, Bob Palazzo and Jesus Avila for the critical reading of the manuscript. H.M is financed by a post-doctoral fellowship from Fundação para a Ciência e a Tecnologia of Portugal. The work in the laboratory of C.E.S. is financed by the FCT of Portugal and the European Union. 


\section{References}

Abrieu, A., J.A. Kahana, K.W. Wood, and D.W. Cleveland. 2000. CENP-E as an essential component of the mitotic checkpoint in vitro. Cell. 102:817-26.

Abrieu, A., L. Magnaghi-Jaulin, J.A. Kahana, M. Peter, A. Castro, S. Vigneron, T. Lorca, D.W. Cleveland, and J.C. Labbe. 2001. Mps1 is a kinetochore-associated kinase essential for the vertebrate mitotic checkpoint. Cell. 106:83-93.

Adams, R.R., Carmena, M. and Earnshaw, W.C. 2001. Chromosomal passengers and the (Aurora) ABCs of mitosis. Trends Cell Biol. 11:49-54.

Adams, R.R., H. Maiato, W.C. Earnshaw, and M. Carmena. 2001. Essential roles of Drosophila inner centromere protein (INCENP) and aurora B in histone H3 phosphorylation, metaphase chromosome alignment, kinetochore disjunction, and chromosome segregation. J Cell Biol. 153:865-80.

Adams, R.R., A.A. Tavares, A. Salzberg, H.J. Bellen, and D.M. Glover. 1998. pavarotti encodes a kinesin-like protein required to organize the central spindle and contractile ring for cytokinesis. Genes Dev. 12:1483-94.

Adams, R.R., S.P. Wheatley, A.M. Gouldsworthy, S.E. Kandels-Lewis, M. Carmena, C. Smythe, D.L. Gerloff, and W.C. Earnshaw. 2000. INCENP binds the Aurora-related kinase AIRK2 and is required to target it to chromosomes, the central spindle and cleavage furrow. Curr Biol. 10:1075-8.

Afshar, K., N.R. Barton, R.S. Hawley, and L.S. Goldstein. 1995a. DNA binding and meiotic chromosomal localization of the Drosophila nod kinesin-like protein. Cell. 81:129-38.

Afshar, K., J. Scholey, and R.S. Hawley. 1995b. Identification of the chromosome localization domain of the Drosophila nod kinesin-like protein. J Cell Biol. 131:83343.

Ahringer, J. 2003. Control of cell polarity and mitotic spindle positioning in animal cells. Current Opinion in Cell Biology. 15:73-81.

Aist, J.R., H. Liang, and M.W. Berns. 1993. Astral and spindle forces in PtK2 cells during anaphase B: a laser microbeam study. J Cell Sci. 104 ( Pt 4):1207-16.

Akhmanova, A., C.C. Hoogenraad, K. Drabek, T. Stepanova, B. Dortland, T. Verkerk, W. Vermeulen, B.M. Burgering, C.I. De Zeeuw, F. Grosveld, and N. Galjart. 2001. Clasps are CLIP-115 and -170 associating proteins involved in the regional regulation of microtubule dynamics in motile fibroblasts. Cell. 104:923-35. 
Allen, C., and G.G. Borisy. 1974. Structural polarity and directional growth of microtubules of Chlamydomonas flagella. J Mol Biol. 90:381-402.

Alsop, G.B., and D. Zhang. 2003. Microtubules are the only structural constituent of the spindle apparatus required for induction of cell cleavage. J Cell Biol. 162:383-90.

Ambrosini, G., C. Adida, and D.C. Altieri. 1997. A novel anti-apoptosis gene, survivin, expressed in cancer and lymphoma. Nat Med. 3:917-921.

Amon, A. 1999. The spindle checkpoint. Curr Opin Genet Dev. 9:69-75.

Andersen, S.S. 1998. Xenopus interphase and mitotic microtubule-associated proteins differentially suppress microtubule dynamics in vitro. Cell Motil Cytoskeleton. 41:202-13.

Andersen, S.S. 2000. Spindle assembly and the art of regulating microtubule dynamics by MAPs and Stathmin/Op18. Trends Cell Biol. 10:261-7.

Andersen, S.S., A.J. Ashford, R. Tournebize, O. Gavet, A. Sobel, A.A. Hyman, and E. Karsenti. 1997. Mitotic chromatin regulates phosphorylation of Stathmin/Op18. Nature. 389:640-3.

Andersen, S.S., B. Buendia, J.E. Dominguez, A. Sawyer, and E. Karsenti. 1994. Effect on microtubule dynamics of XMAP230, a microtubule-associated protein present in Xenopus laevis eggs and dividing cells. J Cell Biol. 127:1289-99.

Andersen, S.S., and T. Wittmann. 2002. Toward reconstitution of in vivo microtubule dynamics in vitro. Bioessays. 24:305-7.

Ando, A., Y.Y. Kikuti, H. Kawata, N. Okamoto, T. Imai, T. Eki, K. Yokoyama, E. Soeda, T. Ikemura, K. Abe, and et al. 1994. Cloning of a new kinesin-related gene located at the centromeric end of the human MHC region. Immunogenetics. 39:194-200.

Andreassen, P.R., D.K. Palmer, M.H. Wener, and R.L. Margolis. 1991. Telophase disc: a new mammalian mitotic organelle that bisects telophase cells with a possible function in cytokinesis. J Cell Sci. 99 ( Pt 3):523-34.

Andrews, P.D., Y. Ovechkina, N. Morrice, M. Wagenbach, K. Duncan, L. Wordeman, and J.R. Swedlow. 2004. Aurora B regulates MCAK at the mitotic centromere. Dev Cell. 6:253-68.

Antonio, C., I. Ferby, H. Wilhelm, M. Jones, E. Karsenti, A.R. Nebreda, and I. Vernos. 2000. Xkid, a chromokinesin required for chromosome alignment on the metaphase plate. Cell. 102:425-35. 
Arnal, I., E. Karsenti, and A.A. Hyman. 2000. Structural transitions at microtubule ends correlate with their dynamic properties in Xenopus egg extracts. J Cell Biol. 149:76774.

Arnaud, L., J. Pines, and E.A. Nigg. 1998. GFP tagging reveals human Polo-like kinase 1 at the kinetochore/centromere region of mitotic chromosomes. Chromosoma. 107:424-9.

Askham, J.M., P. Moncur, A.F. Markham, and E.E. Morrison. 2000. Regulation and function of the interaction between the APC tumour suppressor protein and EB1. Oncogene. 19:1950-8.

Askham, J.M., K.T. Vaughan, H.V. Goodson, and E.E. Morrison. 2002. Evidence That an Interaction between EB1 and p150Glued Is Required for the Formation and Maintenance of a Radial Microtubule Array Anchored at the Centrosome. Mol. Biol. Cell. 13:3627-3645.

Ault, J.G., and C.L. Rieder. 1994. Centrosome and kinetochore movement during mitosis. Curr Opin Cell Biol. 6:41-9.

Bajer, A.S. 1982. Functional autonomy of monopolar spindle and evidence for oscillatory movement in mitosis. J Cell Biol. 93:33-48.

Bajer, A.S., and J. Mole-Bajer. 1972. Spindle dynamics and chromosome movements. Int. Rev. Cytol. 34:1-271.

Bajer, A.S., and M. Vantard. 1988. Microtubule dynamics determine chromosome lagging and transport of acentric fragments. Mutat Res. 201:271-81.

Balczon, R.D., and B.R. Brinkley. 1987. Tubulin interaction with kinetochore proteins: analysis by in vitro assembly and chemical cross-linking. J Cell Biol. 105:855-62.

Banks, J.D., and R. Heald. 2001. Chromosome movement: dynein-out at the kinetochore. Curr Biol. 11:R128-31.

Barton, N.R., and L.S. Goldstein. 1996. Going mobile: microtubule motors and chromosome segregation. Proc Natl Acad Sci U S A. 93:1735-42.

Barton, N.R., A.J. Pereira, and L.S. Goldstein. 1995. Motor activity and mitotic spindle localization of the Drosophila kinesin-like protein KLP61F. Mol Biol Cell. 6:1563-74.

Basto, R., R. Gomes, and R.E. Karess. 2000. Rough deal and Zw10 are required for the metaphase checkpoint in Drosophila. Nat Cell Biol. 2:939-43.

Basu, J., H. Bousbaa, E. Logarinho, Z. Li, B.C. Williams, C. Lopes, C.E. Sunkel, and M.L. Goldberg. 1999. Mutations in the essential spindle checkpoint gene bub1 cause chromosome missegregation and fail to block apoptosis in Drosophila. J Cell Biol. 146:13-28. 
Basu, J., E. Logarinho, S. Herrmann, H. Bousbaa, Z. Li, G.K. Chan, T.J. Yen, C.E. Sunkel, and M.L. Goldberg. 1998. Localization of the Drosophila checkpoint control protein Bub3 to the kinetochore requires Bub1 but not Zw10 or Rod. Chromosoma. 107:37685.

Beach, D.L., J. Thibodeaux, P. Maddox, E. Yeh, and K. Bloom. 2000. The role of the proteins Kar9 and Myo2 in orienting the mitotic spindle of budding yeast. Curr Biol. 10:1497-506.

Beaudouin, J., D. Gerlich, N. Daigle, R. Eils, and J. Ellenberg. 2002. Nuclear envelope breakdown proceeds by microtubule-induced tearing of the lamina. Cell. 108:83-96.

Beinhauer, J.D., I.M. Hagan, J.H. Hegemann, and U. Fleig. 1997. Mal3, the fission yeast homologue of the human APC-interacting protein EB-1 is required for microtubule integrity and the maintenance of cell form. J Cell Biol. 139:717-28.

Bellanger, J.M., and P. Gonczy. 2003. TAC-1 and ZYG-9 form a complex that promotes microtubule assembly in C. elegans embryos. Curr Biol. 13:1488-98.

Belmont, L.D., A.A. Hyman, K.E. Sawin, and T.J. Mitchison. 1990. Real-time visualization of cell cycle-dependent changes in microtubule dynamics in cytoplasmic extracts. Cell. 62:579-89.

Belmont, L.D., and T.J. Mitchison. 1996. Identification of a protein that interacts with tubulin dimers and increases the catastrophe rate of microtubules. Cell. 84:623-31.

Berlin, V., C.A. Styles, and G.R. Fink. 1990. BIK1, a protein required for microtubule function during mating and mitosis in Saccharomyces cerevisiae, colocalizes with tubulin. J Cell Biol. 111:2573-86.

Bernat, R. L., Borisy, G. G., Rothfield, N. F. and Earnshaw, W. C. 1990. Injection of anticentromere antibodies in interphase disrupts events required for chromosome movement at mitosis. J Cell Biol. 111:1519-33.

Berns, M.W., and S.M. Richardson. 1977. Continuation of mitosis after selective laser microbeam destruction of the centriolar region. J Cell Biol. 75:977-82.

Berrueta, L., S.K. Kraeft, J.S. Tirnauer, S.C. Schuyler, L.B. Chen, D.E. Hill, D. Pellman, and B.E. Bierer. 1998. The adenomatous polyposis coli-binding protein EB1 is associated with cytoplasmic and spindle microtubules. Proc Natl Acad Sci U S A. 95:10596-601.

Berrueta, L., J.S. Tirnauer, S.C. Schuyler, D. Pellman, and B.E. Bierer. 1999. The APCassociated protein EB1 associates with components of the dynactin complex and cytoplasmic dynein intermediate chain. Curr Biol. 9:425-8. 
Bhattacharjee, R.N., F. Hamada, K. Toyoshima, and T. Akiyama. 1996. The tumor suppressor gene product APC is hyperphosphorylated during the M phase. Biochem Biophys Res Commun. 220:192-5.

Bienz, M. 2002. The subcellular destinations of APC proteins. Nat Rev Mol Cell Biol. 3:32838.

Biggins, S., and A.W. Murray. 2001. The budding yeast protein kinase Ipl1/Aurora allows the absence of tension to activate the spindle checkpoint. Genes Dev. 15:3118-29.

Biggins, S., and C.E. Walczak. 2003. Captivating capture: how microtubules attach to kinetochores. Curr Biol. 13:R449-60.

Bischoff, J.R., and G.D. Plowman. 1999. The Aurora/Ipl1p kinase family: regulators of chromosome segregation and cytokinesis. Trends Cell Biol. 9:454-459.

Blangy, A., L. Arnaud, and E.A. Nigg. 1997. Phosphorylation by p34cdc2 protein kinase regulates binding of the kinesin-related motor HsEg5 to the dynactin subunit p150. $J$ Biol Chem. 272:19418-24.

Blangy, A., H.A. Lane, P. d'Herin, M. Harper, M. Kress, and E.A. Nigg. 1995. Phosphorylation by p34cdc2 regulates spindle association of human Eg5, a kinesinrelated motor essential for bipolar spindle formation in vivo. Cell. 83:1159-69.

Bloom, K. 2000. It's a kar9ochore to capture microtubules. Nat Cell Biol. 2:E96-8.

Boleti, H., E. Karsenti, and I. Vernos. 1996. Xklp2, a novel Xenopus centrosomal kinesin-like protein required for centrosome separation during mitosis. Cell. 84:49-59.

Bolton, M.A., W. Lan, S.E. Powers, M.L. McCleland, J. Kuang, and P.T. Stukenberg. 2002. Aurora B Kinase Exists in a Complex with Survivin and INCENP and Its Kinase Activity Is Stimulated by Survivin Binding and Phosphorylation. Mol Biol Cell. 13:3064-77.

Bonaccorsi, S., M.G. Giansanti, and M. Gatti. 2000. Spindle assembly in Drosophila neuroblasts and ganglion mother cells. Nat Cell Biol. 2:54-6.

Borisy, G.G., J.M. Marcum, J.B. Olmsted, D.B. Murphy, and K.A. Johnson. 1975. Purification of tubulin and associated high molecular weight proteins from porcine brain and characterization of microtubule assembly in vitro. Ann N Y Acad Sci. 253:107-32.

Borisy, G.G., and J.B. Olmsted. 1972. Nucleated assembly of microtubules in porcine brain extracts. Science. 177:1196-7.

Borisy, G.G., and E.W. Taylor. 1967a. The mechanism of action of colchicine. Binding of colchincine-3H to cellular protein. J Cell Biol. 34:525-33. 
Borisy, G.G., and E.W. Taylor. 1967b. The mechanism of action of colchicine. Colchicine binding to sea urchin eggs and the mitotic apparatus. J Cell Biol. 34:535-48.

Bornens, M. 2002. Centrosome composition and microtubule anchoring mechanisms. Curr Opin Cell Biol. 14:25-34.

Brattsand, G., U. Marklund, K. Nylander, G. Roos, and M. Gullberg. 1994. Cell-cycleregulated phosphorylation of oncoprotein 18 on Ser16, Ser25 and Ser38. Eur J Biochem. 220:359-68.

Brinkley, B.R., and E. Stubblefield. 1966. The fine structure of the kinetochore of a mammalian cell in vitro. Chromosoma. 19:28-43.

Brinkley, B.R., R.P. Zinkowski, W.L. Mollon, F.M. Davis, M.A. Pisegna, M. Pershouse, and P.N. Rao. 1988. Movement and segregation of kinetochores experimentally detached from mammalian chromosomes. Nature. 336:251-4.

Brunner, D., and P. Nurse. 2000. CLIP170-like tip1p spatially organizes microtubular dynamics in fission yeast. Cell. 102:695-704.

Bu, W., and L.K. Su. 2001. Regulation of microtubule assembly by human EB1 family proteins. Oncogene. 20:3185-92.

Bucciarelli, E., M.G. Giansanti, S. Bonaccorsi, and M. Gatti. 2003. Spindle assembly and cytokinesis in the absence of chromosomes during Drosophila male meiosis. J Cell Biol. 160:993-9.

Budde, P.P., A. Kumagai, W.G. Dunphy, and R. Heald. 2001. Regulation of Op18 during spindle assembly in Xenopus egg extracts. J Cell Biol. 153:149-58.

Bulinski, J.C., and G.G. Borisy. 1980a. Immunofluorescence localization of HeLa cell microtubule-associated proteins on microtubules in vitro and in vivo. J Cell Biol. 87:792-801.

Bulinski, J.C., and G.G. Borisy. 1980b. Microtubule-associated proteins from cultured HeLa cells. Analysis of molecular properties and effects on microtubule polymerization. $J$ Biol Chem. 255:11570-6.

Bulinski, J.C., and G.G. Borisy. 1980c. Widespread distribution of a 210,000 mol wt microtubule-associated protein in cells and tissues of primates. J Cell Biol. 87:802-8.

Busson, S., D. Dujardin, A. Moreau, J. Dompierre, and J.R. De Mey. 1998. Dynein and dynactin are localized to astral microtubules and at cortical sites in mitotic epithelial cells. Curr Biol. 8:541-4.

Buster, D., K. McNally, and F.J. McNally. 2002. Katanin inhibition prevents the redistribution of gamma-tubulin at mitosis. J Cell Sci. 115:1083-92. 
Cahill, D.P., C. Lengauer, J. Yu, G.J. Riggins, J.K. Willson, S.D. Markowitz, K.W. Kinzler, and B. Vogelstein. 1998. Mutations of mitotic checkpoint genes in human cancers. Nature. 392:300-3.

Campbell, M.S., and G.J. Gorbsky. 1995. Microinjection of mitotic cells with the 3F3/2 antiphosphoepitope antibody delays the onset of anaphase. J Cell Biol. 129:1195-204.

Cande, W.Z. 1982. Nucleotide requirements for anaphase chromosome movements in permeabilized mitotic cells: anaphase B but not anaphase A requires ATP. Cell. 28:15-22.

Canman, J.C., L.A. Cameron, P.S. Maddox, A. Straight, J.S. Tirnauer, T.J. Mitchison, G. Fang, T.M. Kapoor, and E.D. Salmon. 2003. Determining the position of the cell division plane. Nature. 424:1074-8.

Canman, J.C., D.B. Hoffman, and E.D. Salmon. 2000. The role of pre- and post-anaphase microtubules in the cytokinesis phase of the cell cycle. Curr Biol. 10:611-4.

Cao, L.G., and Y.L. Wang. 1996. Signals from the spindle midzone are required for the stimulation of cytokinesis in cultured epithelial cells. Mol Biol Cell. 7:225-32.

Caplow, M. 1992. Microtubule dynamics. Current Opinion in Cell Biology. 4:58-65.

Caplow, M., and J. Shanks. 1996. Evidence that a single monolayer tubulin-GTP cap is both necessary and sufficient to stabilize microtubules. Mol Biol Cell. 7:663-75.

Carazo-Salas, R.E., G. Guarguaglini, O.J. Gruss, A. Segref, E. Karsenti, and I.W. Mattaj. 1999. Generation of GTP-bound Ran by RCC1 is required for chromatin-induced mitotic spindle formation. Nature. 400:178-81.

Carlier, M.F., T.L. Hill, and Y. Chen. 1984. Interference of GTP hydrolysis in the mechanism of microtubule assembly: an experimental study. Proc Natl Acad Sci U S A. 81:771-5.

Carlier, M.F., and D. Pantaloni. 1981. Kinetic analysis of guanosine 5'-triphosphate hydrolysis associated with tubulin polymerization. Biochemistry. 20:1918-24.

Carmena, M., and W.C. Earnshaw. 2003. The cellular geography of aurora kinases. Nat Rev Mol Cell Biol. 4:842-54.

Carmena, M., M.G. Riparbelli, G. Minestrini, A.M. Tavares, R. Adams, G. Callaini, and D.M. Glover. 1998. Drosophila polo kinase is required for cytokinesis. J Cell Biol. 143:659-71.

Carminati, J.L., and T. Stearns. 1997. Microtubules Orient the Mitotic Spindle in Yeast through Dynein-dependent Interactions with the Cell Cortex. J. Cell Biol. 138:629641. 
Carvalho, A., M. Carmena, C. Sambade, W.C. Earnshaw, and S.P. Wheatley. 2003. Survivin is required for stable checkpoint activation in taxol-treated HeLa cells. J Cell Sci. 116:2987-98.

Cassimeris, L. 2002. The oncoprotein 18/stathmin family of microtubule destabilizers. Curr Opin Cell Biol. 14:18-24.

Cassimeris, L., D. Gard, P.T. Tran, and H.P. Erickson. 2001. XMAP215 is a long thin molecule that does not increase microtubule stiffness. J Cell Sci. 114:3025-33.

Cassimeris, L., N. Pryer, and E. Salmon. 1988. Real-time observations of microtubule dynamic instability in living cells. J. Cell Biol. 107:2223-2231.

Cassimeris, L., C.L. Rieder, and E.D. Salmon. 1994. Microtubule assembly and kinetochore directional instability in vertebrate monopolar spindles: implications for the mechanism of chromosome congression. J Cell Sci. 107 ( Pt 1):285-97.

Cassimeris, L., and E.D. Salmon. 1991. Kinetochore microtubules shorten by loss of subunits at the kinetochores of prometaphase chromosomes. J Cell Sci. 98 ( Pt 2):151-8.

Cha, B., L. Cassimeris, and D.L. Gard. 1999. XMAP230 is required for normal spindle assembly in vivo and in vitro. J Cell Sci. 112 ( Pt 23):4337-46.

Cha, B.J., B. Error, and D.L. Gard. 1998. XMAP230 is required for the assembly and organization of acetylated microtubules and spindles in Xenopus oocytes and eggs. $J$ Cell Sci. 111 ( Pt 16):2315-27.

Chan, G.K., S.A. Jablonski, D.A. Starr, M.L. Goldberg, and T.J. Yen. 2000. Human Zw10 and ROD are mitotic checkpoint proteins that bind to kinetochores. Nat Cell Biol. 2:944-7.

Chan, G.K., B.T. Schaar, and T.J. Yen. 1998. Characterization of the kinetochore binding domain of CENP-E reveals interactions with the kinetochore proteins CENP-F and hBUBR1. J Cell Biol. 143:49-63.

Chang, W., D. Gruber, S. Chari, H. Kitazawa, Y. Hamazumi, S. Hisanaga, and J.C. Bulinski. 2001. Phosphorylation of MAP4 affects microtubule properties and cell cycle progression. J Cell Sci. 114:2879-87.

Charles, J., S. Jaspersen, R. Tinker-Kulberg, L. Hwang, A. Szidon, and D. Morgan. 1998. The Polo-related kinase Cdc5 activates and is destroyed by the mitotic cyclin destruction machinery in S. cerevisiae. Current Biology. 8:497-507.

Charrasse, S., M. Mazel, S. Taviaux, P. Berta, T. Chow, and C. Larroque. 1995. Characterization of the cDNA and pattern of expression of a new gene over-expressed in human hepatomas and colonic tumors. Eur J Biochem. 234:406-13. 
Charrasse, S., M. Schroeder, C. Gauthier-Rouviere, F. Ango, L. Cassimeris, D.L. Gard, and C. Larroque. 1998. The TOGp protein is a new human microtubule-associated protein homologous to the Xenopus XMAP215. J Cell Sci. 111 ( Pt 10):1371-83.

Cheeseman, I.M., S. Anderson, M. Jwa, E.M. Green, J. Kang, J.R. Yates, C.S. Chan, D.G. Drubin, and G. Barnes. 2002. Phospho-regulation of kinetochore-microtubule attachments by the aurora kinase ipl1p. Cell. 111:163-72.

Cheeseman, I.M., M. Enquist-Newman, T. Muller-Reichert, D.G. Drubin, and G. Barnes. 2001. Mitotic spindle integrity and kinetochore function linked by the Duo1p/Dam1p complex. J Cell Biol. 152:197-212.

Chen, J., S. Jin, S.K. Tahir, H. Zhang, X. Liu, A.V. Sarthy, T.P. McGonigal, Z. Liu, S.H. Rosenberg, and S.C. Ng. 2003. Survivin enhances Aurora-B kinase activity and localizes Aurora-B in human cells. J Biol Chem. 278:486-90.

Chen, R.H., J.C. Waters, E.D. Salmon, and A.W. Murray. 1996. Association of spindle assembly checkpoint component XMAD2 with unattached kinetochores. Science. 274:242-6.

Chen, X.P., H. Yin, and T.C. Huffaker. 1998. The yeast spindle pole body component Spc72p interacts with Stu2p and is required for proper microtubule assembly. J Cell Biol. 141:1169-79.

Choi, J.H., P.G. Bertram, R. Drenan, J. Carvalho, H.H. Zhou, and X.F. Zheng. 2002. The FKBP12-rapamycin-associated protein (FRAP) is a CLIP-170 kinase. EMBO Rep. 3:988-94.

Chretien, D., S.D. Fuller, and E. Karsenti. 1995. Structure of growing microtubule ends: twodimensional sheets close into tubes at variable rates. J Cell Biol. 129:1311-28.

Comings, D.E., and T.A. Okada. 1971. Fine structure of kinetochore in Indian muntjac. Exp Cell Res. 67:97-110.

Compton, D. 1998. Focusing on spindle poles. J Cell Sci. 111:1477-1481.

Compton, D.A., and D.W. Cleveland. 1994. NuMA, a nuclear protein involved in mitosis and nuclear reformation. Curr Opin Cell Biol. 6:343-6.

Connolly, J.A., V.I. Kalnins, D.W. Cleveland, and M.W. Kirschner. 1978. Intracellular localization of the high molecular weight microtubule accessory protein by indirect immunofluorescence. J Cell Biol. 76:781-6.

Cooke, C.A., D.P. Bazett-Jones, W.C. Earnshaw, and J.B. Rattner. 1993. Mapping DNA within the mammalian kinetochore. J Cell Biol. 120:1083-91. 
Cooke, C.A., M.M. Heck, and W.C. Earnshaw. 1987. The inner centromere protein (INCENP) antigens: movement from inner centromere to midbody during mitosis. $J$ Cell Biol. 105:2053-67.

Cooke, C.A., B. Schaar, T.J. Yen, and W.C. Earnshaw. 1997. Localization of CENP-E in the fibrous corona and outer plate of mammalian kinetochores from prometaphase through anaphase. Chromosoma. 106:446-55.

Coquelle, F.M., M. Caspi, F.P. Cordelieres, J.P. Dompierre, D.L. Dujardin, C. Koifman, P. Martin, C.C. Hoogenraad, A. Akhmanova, N. Galjart, J.R. De Mey, and O. Reiner. 2002. LIS1, CLIP-170's key to the dynein/dynactin pathway. Mol Cell Biol. 22:3089102.

Corces, V.G., J. Salas, M.L. Salas, and J. Avila. 1978. Binding of microtubule proteins to DNA: specificity of the interaction. Eur J Biochem. 86:473-9.

Cottingham, F.R., L. Gheber, D.L. Miller, and M.A. Hoyt. 1999. Novel roles for saccharomyces cerevisiae mitotic spindle motors. J Cell Biol. 147:335-50.

Cottingham, F.R., and M.A. Hoyt. 1997. Mitotic spindle positioning in Saccharomyces cerevisiae is accomplished by antagonistically acting microtubule motor proteins. $J$ Cell Biol. 138:1041-53.

Coue, M., V. Lombillo, and J. McIntosh. 1991. Microtubule depolymerization promotes particle and chromosome movement in vitro. J. Cell Biol. 112:1165-1175.

Craig, J.M., W.C. Earnshaw, and P. Vagnarelli. 1999. Mammalian centromeres: DNA sequence, protein composition, and role in cell cycle progression. Exp Cell Res. 246:249-62.

Cullen, C.F., P. Deak, D.M. Glover, and H. Ohkura. 1999. mini spindles: A gene encoding a conserved microtubule-associated protein required for the integrity of the mitotic spindle in Drosophila. J Cell Biol. 146:1005-18.

Cullen, C.F., and H. Ohkura. 2001. Msps protein is localized to acentrosomal poles to ensure bipolarity of Drosophila meiotic spindles. Nat Cell Biol. 3:637-42.

Cutts, S.M., K.J. Fowler, B.T. Kile, L.L. Hii, R.A. O'Dowd, D.F. Hudson, R. Saffery, P. Kalitsis, E. Earle, and K.H. Choo. 1999. Defective chromosome segregation, microtubule bundling and nuclear bridging in inner centromere protein gene (Incenp)disrupted mice. Hum Mol Genet. 8:1145-55.

Danilchik, M.V., W.C. Funk, E.E. Brown, and K. Larkin. 1998. Requirement for microtubules in new membrane formation during cytokinesis of Xenopus embryos. Dev Biol. 194:47-60. 
Davis, L.J., D.J. Odde, S.M. Block, and S.P. Gross. 2002. The importance of lattice defects in katanin-mediated microtubule severing in vitro. Biophys J. 82:2916-27.

De Brabander, M., J.C. Bulinski, G. Geuens, J. De Mey, and G.G. Borisy. 1981. Immunoelectron microscopic localization of the 210,000-mol wt microtubuleassociated protein in cultured cells of primates. J Cell Biol. 91:438-45.

De Mey, J., Aerts, F., Moeremans, M., Geuens, G., Daneels, G., and De Brabander, M. 1984. Anti-MAP-1 reacts with the centrosome, kinetochores, midbody and spindle of mitotic Ptk2 cells. J Cell Biol. 99:447a.

De Zeeuw, C.I., C.C. Hoogenraad, E. Goedknegt, E. Hertzberg, A. Neubauer, F. Grosveld, and N. Galjart. 1997. CLIP-115, a novel brain-specific cytoplasmic linker protein, mediates the localization of dendritic lamellar bodies. Neuron. 19:1187-99.

Debec, A., C. Detraves, C. Montmory, G. Geraud, and M. Wright. 1995. Polar organization of gamma-tubulin in acentriolar mitotic spindles of Drosophila melanogaster cells. $J$ Cell Sci. 108 ( Pt 7):2645-53.

Desai, A., P.S. Maddox, T.J. Mitchison, and E.D. Salmon. 1998. Anaphase A chromosome movement and poleward spindle microtubule flux occur At similar rates in Xenopus extract spindles. J Cell Biol. 141:703-13.

Desai, A., and T.J. Mitchison. 1995. A new role for motor proteins as couplers to depolymerizing microtubules. J Cell Biol. 128:1-4.

Desai, A., and T.J. Mitchison. 1997. Microtubule polymerization dynamics. Annu Rev Cell Dev Biol. 13:83-117.

Desai, A., S. Verma, T.J. Mitchison, and C.E. Walczak. 1999. Kin I kinesins are microtubuledestabilizing enzymes. Cell. 96:69-78.

Descombes, P., and E.A. Nigg. 1998. The polo-like kinase Plx1 is required for M phase exit and destruction of mitotic regulators in Xenopus egg extracts. Embo J. 17:1328-35.

DeZwaan, T.M., E. Ellingson, D. Pellman, and D.M. Roof. 1997. Kinesin-related KIP3 of Saccharomyces cerevisiae is required for a distinct step in nuclear migration. J Cell Biol. 138:1023-40.

Diamantopoulos, G.S., F. Perez, H.V. Goodson, G. Batelier, R. Melki, T.E. Kreis, and J.E. Rickard. 1999. Dynamic localization of CLIP-170 to microtubule plus ends is coupled to microtubule assembly. J Cell Biol. 144:99-112.

Dictenberg, J.B., W. Zimmerman, C.A. Sparks, A. Young, C. Vidair, Y. Zheng, W. Carrington, F.S. Fay, and S.J. Doxsey. 1998. Pericentrin and gamma-tubulin form a 
protein complex and are organized into a novel lattice at the centrosome. J Cell Biol. 141:163-74.

Dionne, M.A., L. Howard, and D.A. Compton. 1999. NuMA is a component of an insoluble matrix at mitotic spindle poles. Cell Motil Cytoskeleton. 42:189-203.

Dionne, M.A., A. Sanchez, and D.A. Compton. 2000. ch-TOGp is required for microtubule aster formation in a mammalian mitotic extract. J Biol Chem. 275:12346-52.

Ditchfield, C., V.L. Johnson, A. Tighe, R. Ellston, C. Haworth, T. Johnson, A. Mortlock, N. Keen, and S.S. Taylor. 2003. Aurora B couples chromosome alignment with anaphase by targeting BubR1, Mad2, and Cenp-E to kinetochores. J. Cell Biol. 161:267-280.

do Carmo Avides, M., A. Tavares, and D.M. Glover. 2001. Polo kinase and Asp are needed to promote the mitotic organizing activity of centrosomes. Nat Cell Biol. 3:421-4.

Dobles, M., V. Liberal, M.L. Scott, R. Benezra, and P.K. Sorger. 2000. Chromosome missegregation and apoptosis in mice lacking the mitotic checkpoint protein Mad2. Cell. 101:635-45.

Doxsey, S. 2001. Re-evaluating centrosome function. Nat Rev Mol Cell Biol. 2:688-98.

Doxsey, S.J., P. Stein, L. Evans, P.D. Calarco, and M. Kirschner. 1994. Pericentrin, a highly conserved centrosome protein involved in microtubule organization. Cell. 76:639-50.

Drechsel, D.N., and M.W. Kirschner. 1994. The minimum GTP cap required to stabilize microtubules. Curr Biol. 4:1053-61.

Dujardin, D., U.I. Wacker, A. Moreau, T.A. Schroer, J.E. Rickard, and J.R. De Mey. 1998. Evidence for a role of CLIP-170 in the establishment of metaphase chromosome alignment. J Cell Biol. 141:849-62.

Dujardin, D.L., and R.B. Vallee. 2002. Dynein at the cortex. Curr Opin Cell Biol. 14:44-9.

Earnshaw, W.C. 1994. Structure and molecular biology of the kinetochore. In Microtubules. Wiley-Liss, Inc. 393-412.

Earnshaw, W.C. 2001. Chromosomal passengers. Curr Biol. 11:R683.

Earnshaw, W.C., and R.L. Bernat. 1991. Chromosomal passengers: toward an integrated view of mitosis. Chromosoma. 100:139-46.

Earnshaw, W.C., and C.A. Cooke. 1991. Analysis of the distribution of the INCENPs throughout mitosis reveals the existence of a pathway of structural changes in the chromosomes during metaphase and early events in cleavage furrow formation. J Cell Sci. 98 ( Pt 4):443-61.

Echeverri, C.J., B.M. Paschal, K.T. Vaughan, and R.B. Vallee. 1996. Molecular characterization of the $50-\mathrm{kD}$ subunit of dynactin reveals function for the complex in 
chromosome alignment and spindle organization during mitosis. J Cell Biol. 132:61733.

Eckley, D.M., A.M. Ainsztein, A.M. Mackay, I.G. Goldberg, and W.C. Earnshaw. 1997. Chromosomal proteins and cytokinesis: patterns of cleavage furrow formation and inner centromere protein positioning in mitotic heterokaryons and mid-anaphase cells. J Cell Biol. 136:1169-83.

Endow, S.A., S. Henikoff, and L. Soler-Niedziela. 1990. Mediation of meiotic and early mitotic chromosome segregation in Drosophila by a protein related to kinesin. Nature. 345:81-3.

Enquist-Newman, M., I.M. Cheeseman, D. Van Goor, D.G. Drubin, P.B. Meluh, and G. Barnes. 2001. Dad1p, third component of the Duo1p/Dam1p complex involved in kinetochore function and mitotic spindle integrity. Mol Biol Cell. 12:2601-13.

Erickson, H.P. 2000. Gamma-tubulin nucleation: template or protofilament? Nat Cell Biol. 2:E93-6.

Erickson, H.P., and D. Stoffler. 1996. Protofilaments and rings, two conformations of the tubulin family conserved from bacterial FtsZ to alpha/beta and gamma tubulin. J Cell Biol. 135:5-8.

Eyers, P.A., E. Erikson, L.G. Chen, and J.L. Maller. 2003. A novel mechanism for activation of the protein kinase aurora a. Curr Biol. 13:691-7.

Farrell, K.W., M.A. Jordan, H.P. Miller, and L. Wilson. 1987. Phase dynamics at microtubule ends: the coexistence of microtubule length changes and treadmilling. J Cell Biol. 104:1035-46.

Faruki, S., R.W. Cole, and C.L. Rieder. 2002. Separating centrosomes interact in the absence of associated chromosomes during mitosis in cultured vertebrate cells. Cell Motil Cytoskeleton. 52:107-21.

Fearnhead, N.S., M.P. Britton, and W.F. Bodmer. 2001. The ABC of APC. Hum Mol Genet. 10:721-33.

Field, C., R. Li, and K. Oegema. 1999. Cytokinesis in eukaryotes: a mechanistic comparison. Curr Opin Cell Biol. 11:68-80.

Fisk, H.A., and M. Winey. 2001. The mouse Mps1p-like kinase regulates centrosome duplication. Cell. 106:95-104.

Fodde, R., J. Kuipers, C. Rosenberg, R. Smits, M. Kielman, C. Gaspar, J.H. van Es, C. Breukel, J. Wiegant, R.H. Giles, and H. Clevers. 2001. Mutations in the APC tumour suppressor gene cause chromosomal instability. Nat Cell Biol. 3:433-8. 
Francis, S.E., and T.N. Davis. 2000. The spindle pole body of Saccharomyces cerevisiae: architecture and assembly of the core components. Curr Top Dev Biol. 49:105-32.

Francisco, L., and C.S. Chan. 1994. Regulation of yeast chromosome segregation by Ipl1 protein kinase and type 1 protein phosphatase. Cell Mol Biol Res. 40:207-13.

Francisco, L., W. Wang, and C.S. Chan. 1994. Type 1 protein phosphatase acts in opposition to IpL1 protein kinase in regulating yeast chromosome segregation. Mol Cell Biol. 14:4731-40.

Fraser, A.G., C. James, G.I. Evan, and M.O. Hengartner. 1999. Caenorhabditis elegans inhibitor of apoptosis protein IAP homologue BIR-1 plays a conserved role in cytokinesis. Curr Biol. 9:292-301.

Fraser, A.G., James, C., Evan, G.I. and Hengartner, M.O. 1999. Caernorhabditis elegans inhibitor of apoptosis (IAP) homologue BIR-1 plays a conserved role in cytokinesis. Curr Biol:292-301.

Fujiwara, K., M.E. Porter, and T.D. Pollard. 1978. Alpha-actinin localization in the cleavage furrow during cytokinesis. J Cell Biol. 79:268-75.

Funabiki, H., and A.W. Murray. 2000. The Xenopus chromokinesin Xkid is essential for metaphase chromosome alignment and must be degraded to allow anaphase chromosome movement. Cell. 102:411-24.

Gaglio, T., M.A. Dionne, and D.A. Compton. 1997. Mitotic spindle poles are organized by structural and motor proteins in addition to centrosomes. J Cell Biol. 138:1055-66.

Gaglio, T., A. Saredi, and D.A. Compton. 1995. NuMA is required for the organization of microtubules into aster-like mitotic arrays. J Cell Biol. 131:693-708.

Garcia, M.A., N. Koonrugsa, and T. Toda. 2002a. Spindle-kinetochore attachment requires the combined action of Kin I-like Klp5/6 and Alp14/Dis1-MAPs in fission yeast. EMBO J. 21:6015-6024.

Garcia, M.A., N. Koonrugsa, and T. Toda. 2002b. Two kinesin-like Kin I family proteins in fission yeast regulate the establishment of metaphase and the onset of anaphase A. Curr Biol. 12:610-21.

Garcia, M.A., L. Vardy, N. Koonrugsa, and T. Toda. 2001. Fission yeast ch-TOG/XMAP215 homologue Alp14 connects mitotic spindles with the kinetochore and is a component of the Mad2-dependent spindle checkpoint. Embo J. 20:3389-401.

Gard, D., and M. Kirschner. 1987. A microtubule-associated protein from Xenopus eggs that specifically promotes assembly at the plus-end. J. Cell Biol. 105:2203-2215. 
Garrett, S., K. Auer, D.A. Compton, and T.M. Kapoor. 2002. hTPX2 Is Required for Normal Spindle Morphology and Centrosome Integrity during Vertebrate Cell Division. Curr Biol. 12:2055-9.

Gatti, M., M.G. Giansanti, and S. Bonaccorsi. 2000. Relationships between the central spindle and the contractile ring during cytokinesis in animal cells. Microsc Res Tech. 49:202-8.

Geiser, J.R., E.J. Schott, T.J. Kingsbury, N.B. Cole, L.J. Totis, G. Bhattacharyya, L. He, and M.A. Hoyt. 1997. Saccharomyces cerevisiae genes required in the absence of the CIN8-encoded spindle motor act in functionally diverse mitotic pathways. Mol Biol Cell. 8:1035-50.

Gergely, F. 2002. Centrosomal TACCtics. Bioessays. 24:915-25.

Gergely, F., V.M. Draviam, and J.W. Raff. 2003. The ch-TOG/XMAP215 protein is essential for spindle pole organization in human somatic cells. Genes Dev. 17:336-41.

Gergely, F., C. Karlsson, I. Still, J. Cowell, J. Kilmartin, and J.W. Raff. 2000a. The TACC domain identifies a family of centrosomal proteins that can interact with microtubules. PNAS. 97:14352-14357.

Gergely, F., D. Kidd, K. Jeffers, J.G. Wakefield, and J.W. Raff. 2000b. D-TACC: a novel centrosomal protein required for normal spindle function in the early Drosophila embryo. Embo J. 19:241-52.

Giansanti, M.G., S. Bonaccorsi, B. Williams, E.V. Williams, C. Santolamazza, M.L. Goldberg, and M. Gatti. 1998. Cooperative interactions between the central spindle and the contractile ring during Drosophila cytokinesis. Genes Dev. 12:396-410.

Gibbons, I.R., and A.J. Rowe. 1965. Dynein: a protein with adenosine triphosphatase activity from cilia. Science. 149:424-426.

Giet, R., and D.M. Glover. 2001. Drosophila aurora B kinase is required for histone H3 phosphorylation and condensin recruitment during chromosome condensation and to organize the central spindle during cytokinesis. J Cell Biol. 152:669-82.

Giet, R., D. McLean, S. Descamps, M.J. Lee, J.W. Raff, C. Prigent, and D.M. Glover. 2002. Drosophila Aurora A kinase is required to localize D-TACC to centrosomes and to regulate astral microtubules. J Cell Biol. 156:437-51.

Giet, R., and C. Prigent. 1999a. Aurora/Ipl1p-related kinases, a new oncogenic family of mitotic serine-threonine kinases. J. Cell Sci. 112:3591-3601.

Giet, R., and C. Prigent. 1999b. Aurora/Ipl1p-related kinases, a new oncogenic family of mitotic serine-threonine kinases. J Cell Sci. 112 ( Pt 21):3591-601. 
Giet, R., R. Uzbekov, F. Cubizolles, K. Le Guellec, and C. Prigent. 1999. The Xenopus laevis aurora-related protein kinase pEg2 associates with and phosphorylates the kinesinrelated protein XlEg5. J Biol Chem. 274:15005-13.

Gillett, E.S., and P.K. Sorger. 2001. Tracing the pathway of spindle assembly checkpoint signaling. Dev Cell. 1:162-4.

Glotzer, M. 2001. Animal cell cytokinesis. Annu Rev Cell Dev Biol. 17:351-86.

Glover, D.M., M.H. Leibowitz, D.A. McLean, and H. Parry. 1995. Mutations in aurora prevent centrosome separation leading to the formation of monopolar spindles. Cell. 81:95-105.

Goldstein, L.S., R.A. Laymon, and J.R. McIntosh. 1986. A microtubule-associated protein in Drosophila melanogaster: identification, characterization, and isolation of coding sequences. J Cell Biol. 102:2076-87.

Golsteyn, R.M., K.E. Mundt, A.M. Fry, and E.A. Nigg. 1995. Cell cycle regulation of the activity and subcellular localization of Plk1, a human protein kinase implicated in mitotic spindle function. J Cell Biol. 129:1617-28.

Gomes, R., R.E. Karess, H. Ohkura, D.M. Glover, and C.E. Sunkel. 1993. Abnormal anaphase resolution (aar): a locus required for progression through mitosis in Drosophila. J Cell Sci. 104 ( Pt 2):583-93.

Gonczy, P., S. Pichler, M. Kirkham, and A.A. Hyman. 1999. Cytoplasmic dynein is required for distinct aspects of MTOC positioning, including centrosome separation, in the one cell stage Caenorhabditis elegans embryo. J Cell Biol. 147:135-50.

Gonzalez, C., G. Tavosanis, and C. Mollinari. 1998. Centrosomes and microtubule organisation during Drosophila development. J Cell Sci. 111 ( Pt 18):2697-706.

Gorbsky, G.J., R.H. Chen, and A.W. Murray. 1998. Microinjection of antibody to Mad2 protein into mammalian cells in mitosis induces premature anaphase. J Cell Biol. 141:1193-205.

Gorbsky, G.J., and W.A. Ricketts. 1993. Differential expression of a phosphoepitope at the kinetochores of moving chromosomes. J Cell Biol. 122:1311-21.

Gorbsky, G.J., P.J. Sammak, and G.G. Borisy. 1987. Chromosomes move poleward in anaphase along stationary microtubules that coordinately disassemble from their kinetochore ends. J Cell Biol. 104:9-18.

Gorbsky, G.J., P.J. Sammak, and G.G. Borisy. 1988. Microtubule dynamics and chromosome motion visualized in living anaphase cells. J Cell Biol. 106:1185-92. 
Gordon, M.B., L. Howard, and D.A. Compton. 2001. Chromosome movement in mitosis requires microtubule anchorage at spindle poles. J Cell Biol. 152:425-34.

Goshima, G., T. Kiyomitsu, K. Yoda, and M. Yanagida. 2003. Human centromere chromatin protein hMis12, essential for equal segregation, is independent of CENP-A loading pathway. J Cell Biol. 160:25-39.

Graf, R., C. Daunderer, and M. Schliwa. 2000. Dictyostelium DdCP224 is a microtubuleassociated protein and a permanent centrosomal resident involved in centrosome duplication. J Cell Sci. 113 ( Pt 10):1747-58.

Grancell, A., and P.K. Sorger. 1998. Chromosome movement: kinetochores motor along. Curr Biol. 8:R382-5.

Gruss, O.J., R.E. Carazo-Salas, C.A. Schatz, G. Guarguaglini, J. Kast, M. Wilm, N. Le Bot, I. Vernos, E. Karsenti, and I.W. Mattaj. 2001. Ran induces spindle assembly by reversing the inhibitory effect of importin alpha on TPX2 activity. Cell. 104:83-93.

Gunawardane, R.N., S.B. Lizarraga, C. Wiese, A. Wilde, and Y. Zheng. 2000. gammaTubulin complexes and their role in microtubule nucleation. Curr Top Dev Biol. 49:55-73.

Hagan, I., and M. Yanagida. 1990. Novel potential mitotic motor protein encoded by the fission yeast cut7+ gene. Nature. 347:563-6.

Hagan, I.M., and J. Petersen. 2000. The microtubule organizing centers of Schizosaccharomyces pombe. Curr Top Dev Biol. 49:133-59.

Haren, L., and A. Merdes. 2002. Direct binding of NuMA to tubulin is mediated by a novel sequence motif in the tail domain that bundles and stabilizes microtubules. J Cell Sci. 115:1815-1824.

Hartman, J.J., J. Mahr, K. McNally, K. Okawa, A. Iwamatsu, S. Thomas, S. Cheesman, J. Heuser, R.D. Vale, and F.J. McNally. 1998. Katanin, a microtubule-severing protein, is a novel AAA ATPase that targets to the centrosome using a WD40-containing subunit. Cell. 93:277-87.

Hartman, J.J., and R.D. Vale. 1999. Microtubule disassembly by ATP-dependent oligomerization of the AAA enzyme katanin. Science. 286:782-5.

Hauf, S., R.W. Cole, S. LaTerra, C. Zimmer, G. Schnapp, R. Walter, A. Heckel, J. van Meel, C.L. Rieder, and J.M. Peters. 2003. The small molecule Hesperadin reveals a role for Aurora B in correcting kinetochore-microtubule attachment and in maintaining the spindle assembly checkpoint. J Cell Biol. 161:281-94. 
Hayashi, S., B. Rubinfeld, B. Souza, P. Polakis, E. Wieschaus, and A.J. Levine. 1997. A Drosophila homolog of the tumor suppressor gene adenomatous polyposis coli downregulates beta-catenin but its zygotic expression is not essential for the regulation of Armadillo. Proc Natl Acad Sci U S A. 94:242-7.

Hayden, J.H., S.S. Bowser, and C.L. Rieder. 1990. Kinetochores capture astral microtubules during chromosome attachment to the mitotic spindle: direct visualization in live newt lung cells. J Cell Biol. 111:1039-45.

Hays, T.S., M.E. Porter, M. McGrail, P. Grissom, P. Gosch, M.T. Fuller, and J.R. McIntosh. 1994. A cytoplasmic dynein motor in Drosophila: identification and localization during embryogenesis. J Cell Sci. 107 ( Pt 6):1557-69.

He, X., D.R. Rines, C.W. Espelin, and P.K. Sorger. 2001. Molecular analysis of kinetochoremicrotubule attachment in budding yeast. Cell. 106:195-206.

Heald, R. 2000. A dynamic duo of microtubule modulators. Nat Cell Biol. 2:E11-2.

Heald, R., R. Tournebize, T. Blank, R. Sandaltzopoulos, P. Becker, A. Hyman, and E. Karsenti. 1996. Self-organization of microtubules into bipolar spindles around artificial chromosomes in Xenopus egg extracts. Nature. 382:420-5.

Healy, A.M., S. Zolnierowicz, A.E. Stapleton, M. Goebl, A.A. DePaoli-Roach, and J.R. Pringle. 1991. CDC55, a Saccharomyces cerevisiae gene involved in cellular morphogenesis: identification, characterization, and homology to the B subunit of mammalian type 2A protein phosphatase. Mol Cell Biol. 11:5767-80.

Heck, M., A. Pereira, P. Pesavento, Y. Yannoni, A. Spradling, and L. Goldstein. 1993. The kinesin-like protein KLP61F is essential for mitosis in Drosophila. J. Cell Biol. 123:665-679.

Herrmann, S., I. Amorim, and C.E. Sunkel. 1998. The POLO kinase is required at multiple stages during spermatogenesis in Drosophila melanogaster. Chromosoma. 107:44051.

Hill, T.L. 1985. Theoretical problems related to the attachment of microtubules to kinetochores. Proc Natl Acad Sci U S A. 82:4404-8.

Hill, T.L., and M.F. Carlier. 1983. Steady-state theory of the interference of GTP hydrolysis in the mechanism of microtubule assembly. Proc Natl Acad Sci U S A. 80:7234-8.

Hill, T.L., and Y. Chen. 1984. Phase changes at the end of a microtubule with a GTP cap. Proc Natl Acad Sci U S A. 81:5772-6. 
Hinchcliffe, E.H., C. Li, E.A. Thompson, J.L. Maller, and G. Sluder. 1999. Requirement of Cdk2-cyclin E activity for repeated centrosome reproduction in Xenopus egg extracts. Science. 283:851-4.

Hinchcliffe, E.H., F.J. Miller, M. Cham, A. Khodjakov, and G. Sluder. 2001. Requirement of a centrosomal activity for cell cycle progression through G1 into S phase. Science. 291:1547-50.

Hiramoto, Y. 1971. Analysis of cleavage stimulus by means of micromanipulation of sea urchin eggs. Exp Cell Res. 68:291-8.

Hirokawa, N., Y. Noda, and Y. Okada. 1998. Kinesin and dynein superfamily proteins in organelle transport and cell division. Curr Opin Cell Biol. 10:60-73.

Hirokawa, N., R. Takemura, and S. Hisanaga. 1985. Cytoskeletal architecture of isolated mitotic spindle with special reference to microtubule-associated proteins and cytoplasmic dynein. J Cell Biol. 101:1858-70.

Hoffman, D.B., C.G. Pearson, T.J. Yen, B.J. Howell, and E.D. Salmon. 2001. Microtubuledependent changes in assembly of microtubule motor proteins and mitotic spindle checkpoint proteins at PtK1 kinetochores. Mol Biol Cell. 12:1995-2009.

Hofmann, C., I.M. Cheeseman, B.L. Goode, K.L. McDonald, G. Barnes, and D.G. Drubin. 1998. Saccharomyces cerevisiae Duo1p and Dam1p, novel proteins involved in mitotic spindle function. J Cell Biol. 143:1029-40.

Hoier, E.F., W.A. Mohler, S.K. Kim, and A. Hajnal. 2000. The Caenorhabditis elegans APCrelated gene apr-1 is required for epithelial cell migration and Hox gene expression. Genes Dev. 14:874-86.

Holmfeldt, P., G. Brattsand, and M. Gullberg. 2002. MAP4 counteracts microtubule catastrophe promotion but not tubulin-sequestering activity in intact cells. Curr Biol. 12:1034-9.

Holmfeldt, P., G. Brattsand, and M. Gullberg. 2003. Interphase and monoastral-mitotic phenotypes of overexpressed MAP4 are modulated by free tubulin concentrations. $J$ Cell Sci. 116:3701-11.

Holmfeldt, P., Stenmark, S. and Gullberg, M.2004. Differential functional interplay of TOGp/XMAP215 and the KinI kinesin MCAK during interphase and mitosis. EMBO J.23: 627-37.

Holy, T.E., and S. Leibler. 1994. Dynamic instability of microtubules as an efficient way to search in space. Proc Natl Acad Sci U S A. 91:5682-5. 
Honda, R., R. Korner, and E.A. Nigg. 2003. Exploring the functional interactions between Aurora B, INCENP, and survivin in mitosis. Mol Biol Cell. 14:3325-41.

Hoogenraad, C.C., A. Akhmanova, F. Grosveld, C.I. De Zeeuw, and N. Galjart. 2000. Functional analysis of CLIP-115 and its binding to microtubules. J Cell Sci. 113 ( Pt 12):2285-97.

Horio, T., and H. Hotani. 1986. Visualization of the dynamic instability of individual microtubules by dark-field microscopy. Nature. 321:605-7.

Hotani, H., and T. Horio. 1988. Dynamics of microtubules visualized by darkfield microscopy: treadmilling and dynamic instability. Cell Motil Cytoskeleton. 10:229-36.

Howell, B., N. Larsson, M. Gullberg, and L. Cassimeris. 1999. Dissociation of the tubulinsequestering and microtubule catastrophe-promoting activities of oncoprotein 18/stathmin. Mol Biol Cell. 10:105-18.

Howell, B.J., D.B. Hoffman, G. Fang, A.W. Murray, and E.D. Salmon. 2000. Visualization of Mad2 dynamics at kinetochores, along spindle fibers, and at spindle poles in living cells. J Cell Biol. 150:1233-50.

Howell, B.J., B.F. McEwen, J.C. Canman, D.B. Hoffman, E.M. Farrar, C.L. Rieder, and E.D. Salmon. 2001. Cytoplasmic dynein/dynactin drives kinetochore protein transport to the spindle poles and has a role in mitotic spindle checkpoint inactivation. J Cell Biol. 155:1159-72.

Hoyt, M.A. 2001. A new view of the spindle checkpoint. J Cell Biol. 154:909-11.

Hoyt, M.A., and J.R. Geiser. 1996. Genetic analysis of the mitotic spindle. Annu Rev Genet. 30:7-33.

Hoyt, M.A., L. He, K.K. Loo, and W.S. Saunders. 1992. Two Saccharomyces cerevisiae kinesin-related gene products required for mitotic spindle assembly. J Cell Biol. 118:109-20.

Hoyt, M.A., L. Totis, and B.T. Roberts. 1991. S. cerevisiae genes required for cell cycle arrest in response to loss of microtubule function. Cell. 66:507-17.

Huitorel, P., and M.W. Kirschner. 1988. The polarity and stability of microtubule capture by the kinetochore. J Cell Biol. 106:151-9.

Hunt, A.J., and J.R. McIntosh. 1998. The dynamic behavior of individual microtubules associated with chromosomes in vitro. Mol Biol Cell. 9:2857-71.

Hunter, A.W., M. Caplow, D.L. Coy, W.O. Hancock, S. Diez, L. Wordeman, and J. Howard. 2003. The kinesin-related protein MCAK is a microtubule depolymerase that forms an ATP-hydrolyzing complex at microtubule ends. Mol Cell. 11:445-57. 
Hyman, A.A. 1995. Microtubule dynamics. Kinetochores get a grip. Curr Biol. 5:483-4.

Hyman, A.A., D. Chretien, I. Arnal, and R.H. Wade. 1995. Structural changes accompanying GTP hydrolysis in microtubules: information from a slowly hydrolyzable analogue guanylyl-(alpha,beta)-methylene-diphosphonate. J Cell Biol. 128:117-25.

Hyman, A.A., and E. Karsenti. 1996. Morphogenetic properties of microtubules and mitotic spindle assembly. Cell. 84:401-10.

Hyman, A.A., and T.J. Mitchison. 1990. Modulation of microtubule stability by kinetochores in vitro. J Cell Biol. 110:1607-16.

Hyman, A.A., S. Salser, D.N. Drechsel, N. Unwin, and T.J. Mitchison. 1992. Role of GTP hydrolysis in microtubule dynamics: information from a slowly hydrolyzable analogue, GMPCPP. Mol Biol Cell. 3:1155-67.

Inoue, S., and E.D. Salmon. 1995. Force generation by microtubule assembly/disassembly in mitosis and related movements. Mol Biol Cell. 6:1619-40.

Inoue, Y.H., M. do Carmo Avides, M. Shiraki, P. Deak, M. Yamaguchi, Y. Nishimoto, A. Matsukage, and D.M. Glover. 2000. Orbit, a novel microtubule-associated protein essential for mitosis in Drosophila melanogaster. J Cell Biol. 149:153-66.

Izant, J.G., J.A. Weatherbee, and J.R. McIntosh. 1982. A microtubule-associated protein in the mitotic spindle and the interphase nucleus. Nature. 295:248-50.

Izant, J.G., J.A. Weatherbee, and J.R. McIntosh. 1983. A microtubule-associated protein antigen unique to mitotic spindle microtubules in PtK1 cells. J Cell Biol. 96:424-34.

Jablonski, S.A., G.K. Chan, C.A. Cooke, W.C. Earnshaw, and T.J. Yen. 1998. The hBUB1 and hBUBR1 kinases sequentially assemble onto kinetochores during prophase with hBUBR1 concentrating at the kinetochore plates in mitosis. Chromosoma. 107:38696.

Janke, C., J. Ortiz, T.U. Tanaka, J. Lechner, and E. Schiebel. 2002. Four new subunits of the Dam1-Duo1 complex reveal novel functions in sister kinetochore biorientation. EMBO J. 21:181-193.

Jiang, W., G. Jimenez, N.J. Wells, T.J. Hope, G.M. Wahl, T. Hunter, and R. Fukunaga. 1998. PRC1: a human mitotic spindle-associated CDK substrate protein required for cytokinesis. Mol Cell. 2:877-85.

Jin, D.Y., F. Spencer, and K.T. Jeang. 1998. Human T cell leukemia virus type 1 oncoprotein Tax targets the human mitotic checkpoint protein MAD1. Cell. 93:81-91.

Johansen, K.M., and J. Johansen. 2002. Recent glimpses of the elusive spindle matrix. Cell Cycle. 1:312-4. 
Jokelainen, P.T. 1967. The ultrastructure and spatial organization of the metaphase kinetochore in mitotic rat cells. J Ultrastruct Res. 19:19-44.

Jones, M.H., J.B. Bachant, A.R. Castillo, T.H. Giddings, Jr., and M. Winey. 1999. Yeast Dam1p is required to maintain spindle integrity during mitosis and interacts with the Mps1p kinase. Mol Biol Cell. 10:2377-91.

Jones, M.H., X. He, T.H. Giddings, and M. Winey. 2001. Yeast Dam1p has a role at the kinetochore in assembly of the mitotic spindle. Proc Natl Acad Sci U S A. 98:1367580.

Jordan, M.A., and L. Wilson. 1998. Use of drugs to study role of microtubule assembly dynamics in living cells. Methods Enzymol. 298:252-76.

Julian, M., Y. Tollon, I. Lajoie-Mazenc, A. Moisand, H. Mazarguil, A. Puget, and M. Wright. 1993. gamma-Tubulin participates in the formation of the midbody during cytokinesis in mammalian cells. J Cell Sci. 105 ( Pt 1):145-56.

Kaitna, S., M. Mendoza, V. Jantsch-Plunger, and M. Glotzer. 2000. Incenp and an aurora-like kinase form a complex essential for chromosome segregation and efficient completion of cytokinesis. Curr Biol. 10:1172-81.

Kaitna, S., P. Pasierbek, M. Jantsch, J. Loidl, and M. Glotzer. 2002. The aurora B kinase AIR-2 regulates kinetochores during mitosis and is required for separation of homologous Chromosomes during meiosis. Curr Biol. 12:798-812.

Kallio, M.J., M.L. McCleland, P.T. Stukenberg, and G.J. Gorbsky. 2002. Inhibition of aurora B kinase blocks chromosome segregation, overrides the spindle checkpoint, and perturbs microtubule dynamics in mitosis. Curr Biol. 12:900-5.

Kaltschmidt, J.A., and A.H. Brand. 2002. Asymmetric cell division: microtubule dynamics and spindle asymmetry. J Cell Sci. 115:2257-2264.

Kang, J., I.M. Cheeseman, G. Kallstrom, S. Velmurugan, G. Barnes, and C.S. Chan. 2001. Functional cooperation of Dam1, Ipl1, and the inner centromere protein (INCENP)related protein Sli15 during chromosome segregation. J Cell Biol. 155:763-74.

Kaplan, K.B., A.A. Burds, J.R. Swedlow, S.S. Bekir, P.K. Sorger, and I.S. Nathke. 2001. A role for the Adenomatous Polyposis Coli protein in chromosome segregation. Nat Cell Biol. 3:429-32.

Karki, S., and E.L. Holzbaur. 1999. Cytoplasmic dynein and dynactin in cell division and intracellular transport. Curr Opin Cell Biol. 11:45-53. 
Karki, S., B. LaMonte, and E.L. Holzbaur. 1998. Characterization of the p22 subunit of dynactin reveals the localization of cytoplasmic dynein and dynactin to the midbody of dividing cells. J Cell Biol. 142:1023-34.

Karsenti, E., and I. Vernos. 2001. The mitotic spindle: a self-made machine. Science. 294:543-7.

Kashina, A.S., G.C. Rogers, and J.M. Scholey. 1997. The bimC family of kinesins: essential bipolar mitotic motors driving centrosome separation. Biochim Biophys Acta. 1357:257-71.

Keating, T.J., and G.G. Borisy. 2000. Immunostructural evidence for the template mechanism of microtubule nucleation. Nat Cell Biol. 2:352-7.

Keller, T.C., 3rd, and L.I. Rebhun. 1982. Strongylocentrotus purpuratus spindle tubulin. I. Characteristics of its polymerization and depolymerization in vitro. J Cell Biol. 93:788-96.

Kellogg, D.R., C.M. Field, and B.M. Alberts. 1989. Identification of microtubule-associated proteins in the centrosome, spindle, and kinetochore of the early Drosophila embryo. $J$ Cell Biol. 109:2977-91.

Kellogg, D.R., M. Moritz, and B.M. Alberts. 1994. The centrosome and cellular organization. Annu Rev Biochem. 63:639-74.

Kemphues, K.J., N. Wolf, W.B. Wood, and D. Hirsh. 1986. Two loci required for cytoplasmic organization in early embryos of Caenorhabditis elegans. Dev Biol. 113:449-60.

Khodjakov, A., R.W. Cole, B.F. McEwen, K.F. Buttle, and C.L. Rieder. 1997. Chromosome fragments possessing only one kinetochore can congress to the spindle equator. J Cell Biol. 136:229-40.

Khodjakov, A., R.W. Cole, B.R. Oakley, and C.L. Rieder. 2000. Centrosome-independent mitotic spindle formation in vertebrates. Curr Biol. 10:59-67.

Khodjakov, A., and C.L. Rieder. 1996. Kinetochores moving away from their associated pole do not exert a significant pushing force on the chromosome. J Cell Biol. 135:315-27.

Khodjakov, A., and C.L. Rieder. 2001. Centrosomes enhance the fidelity of cytokinesis in vertebrates and are required for cell cycle progression. J Cell Biol. 153:237-42.

Kiefer, B., H. Sakai, A.J. Solari, and D. Mazia. 1966. The molecular unit of the microtubules of the mitotic apparatus. $J$ Mol Biol. 20:75-9.

Kim, I.G., D.Y. Jun, U. Sohn, and Y.H. Kim. 1997. Cloning and expression of human mitotic centromere-associated kinesin gene. Biochim Biophys Acta. 1359:181-6. 
Kim, J.H., Kang, J.S. and Chan, C.S. 1999. Sli15 associates with ipl1 protein kinase to promote proper chromosome segregation in Saccharomyces cerevisiae. J Cell Biol. 145:1381-1394.

King, J.M., T.S. Hays, and R.B. Nicklas. 2000. Dynein is a transient kinetochore component whose binding is regulated by microtubule attachment, not tension. $J$ Cell Biol. 151:739-48.

King, J. M. and Nicklas, R. B.2000. Tension on chromosomes increases the number of kinetochore microtubules but only within limits. J.Cell Sci. 113: 3815-23.

Kinoshita, K., I. Arnal, A. Desai, D.N. Drechsel, and A.A. Hyman. 2001. Reconstitution of physiological microtubule dynamics using purified components. Science. 294:1340-3.

Kinoshita, K., B. Habermann, and A.A. Hyman. 2002. XMAP215: a key component of the dynamic microtubule cytoskeleton. Trends Cell Biol. 12:267-73.

Kirshner, M. and Mitchison, T. 1986. Beyond self-assembly: from microtubules to morphogenesis. Cell 45: 329-42.

Kitada, K., A. Johnson, L. Johnston, and A. Sugino. 1993. A multicopy supressor gene of the Saccharomyces cereviseae G1 cell cycle mutant gene dbf4 encodes a protein kinase and is identified as CDC5. Molecular and Cellular Biology. 13:4445-4457.

Kitagawa, R., and A.M. Rose. 1999. Components of the spindle-assembly checkpoint are essential in Caenorhabditis elegans. Nat Cell Biol. 1:514-21.

Kline-Smith, S.L., A. Khodjakov, P. Hergert, and C.E. Walczak. 2003. Depletion of Centromeric MCAK Leads to Chromosome Congression and Segregation Defects Due to Improper Kinetochore Attachments. Mol. Biol. Cell:E03-08-0581.

Kline-Smith, S.L., and C.E. Walczak. 2002. The Microtubule-destabilizing Kinesin XKCM1 Regulates Microtubule Dynamic Instability in Cells. Mol Biol Cell. 13:2718-31.

Knoblich, J.A. 2001. Asymmetric cell division during animal development. Nat Rev Mol Cell Biol. 2:11-20.

Komarova, Y.A., A.S. Akhmanova, S.-i. Kojima, N. Galjart, and G.G. Borisy. 2002. Cytoplasmic linker proteins promote microtubule rescue in vivo. J. Cell Biol. 159:589-599.

Kopecka, M., and M. Gabriel. 1998. The aberrant positioning of nuclei and the microtubular cytoskeleton in Saccharomyces cerevisiae due to improper actin function. Microbiology. 144 ( Pt 7):1783-97.

Kosco, K.A., C.G. Pearson, P.S. Maddox, P.J. Wang, I.R. Adams, E.D. Salmon, K. Bloom, and T.C. Huffaker. 2001. Control of microtubule dynamics by Stu2p is essential for 
spindle orientation and metaphase chromosome alignment in yeast. Mol Biol Cell. $12: 2870-80$.

Koshland, D.E., T.J. Mitchison, and M.W. Kirschner. 1988. Polewards chromosome movement driven by microtubule depolymerization in vitro. Nature. 331:499-504.

Kotani, S., S. Tugendreich, M. Fujii, P.M. Jorgensen, N. Watanabe, C. Hoog, P. Hieter, and K. Todokoro. 1998. PKA and MPF-activated polo-like kinase regulate anaphasepromoting complex activity and mitosis progression. Mol Cell. 1:371-80.

Kufer, T.A., H.H. Sillje, R. Korner, O.J. Gruss, P. Meraldi, and E.A. Nigg. 2002. Human TPX2 is required for targeting Aurora-A kinase to the spindle. J Cell Biol. 158:61723.

Kumagai, A., and W.G. Dunphy. 1996. Purification and molecular cloning of Plx1, a Cdc25regulatory kinase from Xenopus egg extracts. Science. 273:1377-80.

Kuntziger, T., O. Gavet, V. Manceau, A. Sobel, and M. Bornens. 2001. Stathmin/Op18 phosphorylation is regulated by microtubule assembly. Mol Biol Cell. 12:437-48.

Kuriyama, R., C. Gustus, Y. Terada, Y. Uetake, and J. Matuliene. 2002. CHO1, a mammalian kinesin-like protein, interacts with F-actin and is involved in the terminal phase of cytokinesis. J. Cell Biol. 156:783-790.

Kuriyama, R., M. Kofron, R. Essner, T. Kato, S. Dragas-Granoic, C.K. Omoto, and A. Khodjakov. 1995. Characterization of a minus end-directed kinesin-like motor protein from cultured mammalian cells. J Cell Biol. 129:1049-59.

Lacey, K.R., P.K. Jackson, and T. Stearns. 1999. Cyclin-dependent kinase control of centrosome duplication. Proc Natl Acad Sci U S A. 96:2817-22.

Lantz, V.A., and K.G. Miller. 1998. A class VI unconventional myosin is associated with a homologue of a microtubule-binding protein, cytoplasmic linker protein-170, in neurons and at the posterior pole of Drosophila embryos. J Cell Biol. 140:897-910.

Larsson, N., U. Marklund, H.M. Gradin, G. Brattsand, and M. Gullberg. 1997. Control of microtubule dynamics by oncoprotein 18: dissection of the regulatory role of multisite phosphorylation during mitosis. Mol Cell Biol. 17:5530-9.

Larsson, N., H. Melander, U. Marklund, O. Osterman, and M. Gullberg. 1995. G2/M transition requires multisite phosphorylation of oncoprotein 18 by two distinct protein kinase systems. J Biol Chem. 270:14175-83.

Larsson, N., B. Segerman, H.M. Gradin, E. Wandzioch, L. Cassimeris, and M. Gullberg. 1999a. Mutations of oncoprotein 18/stathmin identify tubulin-directed regulatory activities distinct from tubulin association. Mol Cell Biol. 19:2242-50. 
Larsson, N., B. Segerman, B. Howell, K. Fridell, L. Cassimeris, and M. Gullberg. 1999b. Op18/stathmin mediates multiple region-specific tubulin and microtubule-regulating activities. J Cell Biol. 146:1289-302.

Le Bot, N., M.C. Tsai, R.K. Andrews, and J. Ahringer. 2003. TAC-1, a regulator of microtubule length in the C. elegans embryo. Curr Biol. 13:1499-505.

Le Guellec, R., J. Paris, A. Couturier, C. Roghi, and M. Philippe. 1991. Cloning by differential screening of a Xenopus cDNA that encodes a kinesin-related protein. $\mathrm{Mol}$ Cell Biol. 11:3395-8.

Lee, K.S., Y.L. Yuan, R. Kuriyama, and R.L. Erikson. 1995. Plk is an M-phase-specific protein kinase and interacts with a kinesin-like protein, CHO1/MKLP-1. Mol Cell Biol. 15:7143-51.

Lee, M.J., F. Gergely, K. Jeffers, S.Y. Peak-Chew, and J.W. Raff. 2001a. Msps/XMAP215 interacts with the centrosomal protein D-TACC to regulate microtubule behaviour. Nat Cell Biol. 3:643-9.

Lee, Y.M., S. Lee, E. Lee, H. Shin, H. Hahn, W. Choi, and W. Kim. 2001b. Human kinesin superfamily member 4 is dominantly localized in the nuclear matrix and is associated with chromosomes during mitosis. Biochem. J. 360:549-556.

Lemos, C.L., P. Sampaio, H. Maiato, M. Costa, L.V. Omel'yanchuk, V. Liberal, and C.E. Sunkel. 2000. Mast, a conserved microtubule-associated protein required for bipolar mitotic spindle organization. Embo J. 19:3668-82.

Lens, S.M., R.M. Wolthuis, R. Klompmaker, J. Kauw, R. Agami, T. Brummelkamp, G. Kops, and R.H. Medema. 2003. Survivin is required for a sustained spindle checkpoint arrest in response to lack of tension. Embo J. 22:2934-47.

Leslie, R.J. 1992. Chromosomes attain a metaphase position on half-spindles in the absence of an opposing spindle pole. J Cell Sci. 103 ( Pt 1):125-30.

Leverson, J.D., H.K. Huang, S.L. Forsburg, and T. Hunter. 2002. The Schizosaccharomyces pombe aurora-related kinase Ark1 interacts with the inner centromere protein Pic1 and mediates chromosome segregation and cytokinesis. Mol Biol Cell. 13:1132-43.

Levesque, A.A., and D.A. Compton. 2001. The chromokinesin Kid is necessary for chromosome arm orientation and oscillation, but not congression, on mitotic spindles. J Cell Biol. 154:1135-46.

Levesque, A.A., L. Howard, M.B. Gordon, and D.A. Compton. 2003. A Functional Relationship between NuMA and Kid Is Involved in Both Spindle Organization and Chromosome Alignment in Vertebrate Cells. Mol Biol Cell. 14:3541-52. 
Li, F., E.J. Ackermann, C.F. Bennett, A.L. Rothermel, J. Plescia, S. Tognin, A. Villa, P.C. Marchisio, and D.C. Altieri. 1999. Pleiotropic cell-division defects and apoptosis induced by interference with survivin function. Nat Cell Biol. 1:461-6.

Li, F., G. Ambrosini, E.Y. Chu, J. Plescia, S. Tognin, P.C. Marchisio, and D.C. Altieri. 1998. Control of apoptosis and mitotic spindle checkpoint by survivin. Nature. 396:580-4.

Li, R., and A.W. Murray. 1991. Feedback control of mitosis in budding yeast. Cell. 66:51931.

Li, X., and R.B. Nicklas. 1995. Mitotic forces control a cell-cycle checkpoint. Nature. 373:630-2.

Li, X., and R.B. Nicklas. 1997. Tension-sensitive kinetochore phosphorylation and the chromosome distribution checkpoint in praying mantid spermatocytes. J Cell Sci. 110 ( Pt 5):537-45.

Li, Y., J. Bachant, A.A. Alcasabas, Y. Wang, J. Qin, and S.J. Elledge. 2002. The mitotic spindle is required for loading of the DASH complex onto the kinetochore. Genes Dev. 16:183-97.

Li, Y., and R. Benezra. 1996. Identification of a human mitotic checkpoint gene: hsMAD2. Science. 274:246-8.

Li, Y.Y., E. Yeh, T. Hays, and K. Bloom. 1993. Disruption of mitotic spindle orientation in a yeast dynein mutant. Proc Natl Acad Sci U S A. 90:10096-100.

Liakopoulos, D., J. Kusch, S. Grava, J. Vogel, and Y. Barral. 2003. Asymmetric loading of Kar9 onto spindle poles and microtubules ensures proper spindle alignment. Cell. 112:561-74.

Liao, H., G. Li, and T.J. Yen. 1994. Mitotic regulation of microtubule cross-linking activity of CENP-E kinetochore protein. Science. 265:394-8.

Ligon, L.A., S.S. Shelly, M. Tokito, and E.L.F. Holzbaur. 2003. The Microtubule Plus-End Proteins EB1 and Dynactin Have Differential Effects on Microtubule Polymerization. Mol. Biol. Cell. 14:1405-1417.

Lin, H., P. de Carvalho, D. Kho, C.Y. Tai, P. Pierre, G.R. Fink, and D. Pellman. 2001. Polyploids require Bik1 for kinetochore-microtubule attachment. J Cell Biol. 155:1173-84.

Liu, Z., T. Xie, and R. Steward. 1999. Lis1, the Drosophila homolog of a human lissencephaly disease gene, is required for germline cell division and oocyte differentiation. Development. 126:4477-4488. 
Llamazares, S., A. Moreira, A. Tavares, C. Girdham, B.A. Spruce, C. Gonzalez, R.E. Karess, D.M. Glover, and C.E. Sunkel. 1991. polo encodes a protein kinase homolog required for mitosis in Drosophila. Genes \& Development. 5:2153-2165.

Logarinho, E., and C.E. Sunkel. 1998. The Drosophila POLO kinase localises to multiple compartments of the mitotic apparatus and is required for the phosphorylation of MPM2 reactive epitopes. J Cell Sci. 111 ( Pt 19):2897-909.

Logarinho, E., Bousbaa, H., Dias, J. M., Lopes, C., Amorim, I., Antunes-Martins, A. and Sunkel, C. E. 2004. Different spindle checkpoint proteins monitor microtubule attachment and tension at kinetochores in Drosophila cells. J.Cell Sci. 117: 1757-71.

Lombillo, V.A., C. Nislow, T.J. Yen, V.I. Gelfand, and J.R. McIntosh. 1995a. Antibodies to the kinesin motor domain and CENP-E inhibit microtubule depolymerizationdependent motion of chromosomes in vitro. J Cell Biol. 128:107-15.

Lombillo, V.A., R.J. Stewart, and J.R. McIntosh. 1995b. Minus-end-directed motion of kinesin-coated microspheres driven by microtubule depolymerization. Nature. 373:161-4.

Lu, B., F. Roegiers, L.Y. Jan, and Y.N. Jan. 2001. Adherens junctions inhibit asymmetric division in the Drosophila epithelium. Nature. 409:522-5.

Lydersen, B.K., and D.E. Pettijohn. 1980. Human-specific nuclear protein that associates with the polar region of the mitotic apparatus: distribution in a human/hamster hybrid cell. Cell. 22:489-99.

Lye, R.J., M.E. Porter, J.M. Scholey, and J.R. McIntosh. 1987. Identification of a microtubule-based cytoplasmic motor in the nematode C. elegans. Cell. 51:309-18.

Mackay, A.M., A.M. Ainsztein, D.M. Eckley, and W.C. Earnshaw. 1998. A dominant mutant of inner centromere protein (INCENP), a chromosomal protein, disrupts prometaphase congression and cytokinesis. J Cell Biol. 140:991-1002.

Maddox, P., A. Straight, P. Coughlin, T.J. Mitchison, and E.D. Salmon. 2003. Direct observation of microtubule dynamics at kinetochores in Xenopus extract spindles: implications for spindle mechanics. J Cell Biol. 162:377-82.

Maiato, H., E.A. Fairley, C.L. Rieder, J.R. Swedlow, C.E. Sunkel, and W.C. Earnshaw. 2003a. Human CLASP1 is an outer kinetochore component that regulates spindle microtubule dynamics. Cell. 113:891-904.

Maiato, H., C.L. Rieder, W.C. Earnshaw, and C.E. Sunkel. 2003b. How Do Kinetochores CLASP Dynamic Microtubules? Cell Cycle. 2:511-4. 
Maiato, H., P. Sampaio, C.L. Lemos, J. Findlay, M. Carmena, W.C. Earnshaw, and C.E. Sunkel. 2002. MAST/Orbit has a role in microtubule-kinetochore attachment and is essential for chromosome alignment and maintenance of spindle bipolarity. J Cell Biol. 157:749-60.

Mandelkow, E.M., and E. Mandelkow. 1986. Cryo-electron microscopy of unstained frozenhydrated microtubules. Methods Enzymol. 134:612-33.

Mandelkow, E.M., E. Mandelkow, and R.A. Milligan. 1991. Microtubule dynamics and microtubule caps: a time-resolved cryo-electron microscopy study. J Cell Biol. 114:977-91.

Maney, T., L.M. Ginkel, A.W. Hunter, and L. Wordeman. 2000. The kinetochore of higher eucaryotes: a molecular view. Int Rev Cytol. 194:67-131.

Maney, T., A.W. Hunter, M. Wagenbach, and L. Wordeman. 1998. Mitotic centromereassociated kinesin is important for anaphase chromosome segregation. J Cell Biol. 142:787-801.

Maney, T., M. Wagenbach, and L. Wordeman. 2001. Molecular Dissection of the Microtubule Depolymerizing Activity of Mitotic Centromere-associated Kinesin. $J$. Biol. Chem. 276:34753-34758.

Mao, Y., A. Abrieu, and D.W. Cleveland. 2003. Activating and silencing the mitotic checkpoint through CENP-E-dependent activation/inactivation of BubR1. Cell. 114:87-98.

Margolis, R.L., and L. Wilson. 1978. Opposite end assembly and disassembly of microtubules at steady state in vitro. Cell. 13:1-8.

Margolis, R.L., and L. Wilson. 1981. Microtubule treadmills--possible molecular machinery. Nature. 293:705-11.

Margolis, R.L., and L. Wilson. 1998. Microtubule treadmilling: what goes around comes around. Bioessays. 20:830-6.

Marklund, U., N. Larsson, H.M. Gradin, G. Brattsand, and M. Gullberg. 1996. Oncoprotein 18 is a phosphorylation-responsive regulator of microtubule dynamics. Embo J. 15:5290-8.

Marklund, U., O. Osterman, H. Melander, A. Bergh, and M. Gullberg. 1994. The phenotype of a "Cdc2 kinase target site-deficient" mutant of oncoprotein 18 reveals a role of this protein in cell cycle control. J Biol Chem. 269:30626-35. 
Martineau-Thuillier, S., P.R. Andreassen, and R.L. Margolis. 1998. Colocalization of TD-60 and INCENP throughout G2 and mitosis: evidence for their possible interaction in signalling cytokinesis. Chromosoma. 107:461-70.

Martinez-Exposito, M.J., K.B. Kaplan, J. Copeland, and P.K. Sorger. 1999. Retention of the BUB3 checkpoint protein on lagging chromosomes. Proc Natl Acad Sci U S A. 96:8493-8.

Mastronarde, D.N., K.L. McDonald, R. Ding, and J.R. McIntosh. 1993. Interpolar spindle microtubules in PTK cells. J Cell Biol. 123:1475-89.

Masuda, H., K.L. McDonald, and W.Z. Cande. 1988. The mechanism of anaphase spindle elongation: uncoupling of tubulin incorporation and microtubule sliding during in vitro spindle reactivation. J Cell Biol. 107:623-33.

Mata, J., and P. Nurse. 1997. tea1 and the microtubular cytoskeleton are important for generating global spatial order within the fission yeast cell. Cell. 89:939-49.

Mathur, J., N. Mathur, B. Kernebeck, B.P. Srinivas, and M. Hulskamp. 2003. A novel localization pattern for an EB1-like protein links microtubule dynamics to endomembrane organization. Curr Biol. 13:1991-7.

Matsumoto, Y., K. Hayashi, and E. Nishida. 1999. Cyclin-dependent kinase 2 (Cdk2) is required for centrosome duplication in mammalian cells. Curr Biol. 9:429-32.

Matthews, L.R., P. Carter, D. Thierry-Mieg, and K. Kemphues. 1998. ZYG-9, a Caenorhabditis elegans protein required for microtubule organization and function, is a component of meiotic and mitotic spindle poles. J Cell Biol. 141:1159-68.

Mazia, D. 1961. Mitosis and the physiology of cell division. In The Cell. Vol. 3. J.B.a.A.E. Mirsky, editor. Academic Press, New York. 77-412.

Mazia, D. 1984. Centrosomes and mitotic poles. Exp Cell Res. 153:1-15.

Mazia, D. 1987. The chromosome cycle and the centrosome cycle in the mitotic cycle. Int Rev Cytol. 100:49-92.

Mazia, D., and Dan, K. 1952. The isolation and biochemical characterization of the mitotic apparatus of dividing cells. Proc. Natl. Acad. Sci. U.S.A. 38:826.

Mazia, D., R.R. Chaffee, and R.M. Iverson. 1961. Adenosine triphosphatase in the mitotic apparatus. Proc Natl Acad Sci U S A. 47:788-90.

McCartney, B.M., and e. al. 1999. Drosophila APC2 is a cytoskeletally-associated protein that regulates wingless signaling in the embryonic epidermis. J. Cell Biol. 146:13031318. 
McCartney, B.M., D.G. McEwen, E. Grevengoed, P. Maddox, A. Bejsovec, and M. Peifer. 2001. Drosophila APC2 and Armadillo participate in tethering mitotic spindles to cortical actin. Nat Cell Biol. 3:933-8.

McDonald, H.B., R.J. Stewart, and L.S. Goldstein. 1990. The kinesin-like ncd protein of Drosophila is a minus end-directed microtubule motor. Cell. 63:1159-65.

McEwen, B.F., J.T. Arena, J. Frank, and C.L. Rieder. 1993. Structure of the colcemid-treated PtK1 kinetochore outer plate as determined by high voltage electron microscopic tomography. J Cell Biol. 120:301-12.

McGrail, M., and T.S. Hays. 1997. The microtubule motor cytoplasmic dynein is required for spindle orientation during germline cell divisions and oocyte differentiation in Drosophila. Development. 124:2409-19.

McIntosh, J.a.L., SC. 1971. The distribution of spindle microtubules during mitosis in cultured human cells. J Cell Biol. 49:468-497.

McIntosh, J.R., W.Z. Cande, and J.A. Snyder. 1975. Structure and physiology of the mammalian mitotic spindle. Soc Gen Physiol Ser. 30:31-76.

McNally, F. 2003. Microtubule dynamics: new surprises from an old MAP. Curr Biol. 13:R597-9.

McNally, F.J. 1996. Modulation of microtubule dynamics during the cell cycle. Curr Opin Cell Biol. 8:23-9.

McNally, F.J. 1999. Microtubule dynamics: Controlling split ends. Curr Biol. 9:R274-6.

McNally, F.J. 2001. Cytoskeleton: CLASPing the end to the edge. Curr Biol. 11:R477-80.

McNally, F.J., K. Okawa, A. Iwamatsu, and R.D. Vale. 1996. Katanin, the microtubulesevering ATPase, is concentrated at centrosomes. J Cell Sci. 109 ( Pt 3):561-7.

McNally, F.J., and S. Thomas. 1998. Katanin is responsible for the M-phase microtubulesevering activity in Xenopus eggs. Mol Biol Cell. 9:1847-61.

McNally, F.J., and R.D. Vale. 1993. Identification of katanin, an ATPase that severs and disassembles stable microtubules. Cell. 75:419-29.

McNally, K.P., O.A. Bazirgan, and F.J. McNally. 2000. Two domains of p80 katanin regulate microtubule severing and spindle pole targeting by p60 katanin. J Cell Sci. 113 ( Pt 9):1623-33.

McNally, K.P., D. Buster, and F.J. McNally. 2002. Katanin-mediated microtubule severing can be regulated by multiple mechanisms. Cell Motil Cytoskeleton. 53:337-49.

Meraldi, P., J. Lukas, A.M. Fry, J. Bartek, and E.A. Nigg. 1999. Centrosome duplication in mammalian somatic cells requires E2F and Cdk2-cyclin A. Nat Cell Biol. 1:88-93. 
Merdes, A., and D.W. Cleveland. 1997. Pathways of spindle pole formation: different mechanisms; conserved components. J Cell Biol. 138:953-6.

Merdes, A., and J. De Mey. 1990. The mechanism of kinetochore-spindle attachment and polewards movement analyzed in PtK2 cells at the prophase-prometaphase transition. Eur J Cell Biol. 53:313-25.

Merdes, A., R. Heald, K. Samejima, W.C. Earnshaw, and D.W. Cleveland. 2000. Formation of spindle poles by dynein/dynactin-dependent transport of NuMA. J Cell Biol. 149:851-62.

Merdes, A., K. Ramyar, J.D. Vechio, and D.W. Cleveland. 1996. A complex of NuMA and cytoplasmic dynein is essential for mitotic spindle assembly. Cell. 87:447-58.

Metzner, R. 1894. Beitrage zur Granulalehre. I. Arch. Anat. Physiol.:309-348.

Michel, L.S., V. Liberal, A. Chatterjee, R. Kirchwegger, B. Pasche, W. Gerald, M. Dobles, P.K. Sorger, V.V. Murty, and R. Benezra. 2001. MAD2 haplo-insufficiency causes premature anaphase and chromosome instability in mammalian cells. Nature. 409:355-9.

Middleton, K., and J. Carbon. 1994. KAR3-encoded kinesin is a minus-end-directed motor that functions with centromere binding proteins (CBF3) on an in vitro yeast kinetochore. Proc Natl Acad Sci U S A. 91:7212-6.

Miller, L.K. 1999. An exegesis of IAPs: salvation and surprises from BIR motifs. Trends Cell Biol. 9:323-328.

Miller, R.K., S.C. Cheng, and M.D. Rose. 2000. Bim1p/Yeb1p mediates the Kar9p-dependent cortical attachment of cytoplasmic microtubules. Mol Biol Cell. 11:2949-59.

Miller, R.K., K.K. Heller, L. Frisen, D.L. Wallack, D. Loayza, A.E. Gammie, and M.D. Rose. 1998. The kinesin-related proteins, Kip2p and Kip3p, function differently in nuclear migration in yeast. Mol Biol Cell. 9:2051-68.

Miller, R.K., and M.D. Rose. 1998. Kar9p is a novel cortical protein required for cytoplasmic microtubule orientation in yeast. J Cell Biol. 140:377-390.

Mimori-Kiyosue, Y., N. Shiina, and S. Tsukita. 2000a. Adenomatous polyposis coli (APC) protein moves along microtubules and concentrates at their growing ends in epithelial cells. J Cell Biol. 148:505-18.

Mimori-Kiyosue, Y., N. Shiina, and S. Tsukita. 2000b. The dynamic behavior of the APCbinding protein EB1 on the distal ends of microtubules. Curr Biol. 10:865-8.

Mimori-Kiyosue, Y., and S. Tsukita. 2001. Where is APC going? J Cell Biol. 154:1105-9. 
Mimori-Kiyosue, Y., and S. Tsukita. 2003. "Search-and-capture" of microtubules through plus-end-binding proteins (+TIPs). J Biochem (Tokyo). 134:321-6.

Minshull, J., A. Straight, A.D. Rudner, A.F. Dernburg, A. Belmont, and A.W. Murray. 1996. Protein phosphatase 2A regulates MPF activity and sister chromatid cohesion in budding yeast. Curr Biol. 6:1609-20.

Mitchison, T., L. Evans, E. Schulze, and M. Kirschner. 1986. Sites of microtubule assembly and disassembly in the mitotic spindle. Cell. 45:515-27.

Mitchison, T., and M. Kirschner. 1984a. Dynamic instability of microtubule growth. Nature. 312:237-42.

Mitchison, T., and M. Kirschner. 1984b. Microtubule assembly nucleated by isolated centrosomes. Nature. 312:232-7.

Mitchison, T.J. 1988. Microtubule dynamics and kinetochore function in mitosis. Annu Rev Cell Biol. 4:527-49.

Mitchison, T.J. 1989. Polewards microtubule flux in the mitotic spindle: evidence from photoactivation of fluorescence. J Cell Biol. 109:637-52.

Mitchison, T.J. 1990. Mitosis. The kinetochore in captivity. Nature. 348:14-5.

Mitchison, T.J. 1993. Localization of an exchangeable GTP binding site at the plus end of microtubules. Science. 261:1044-7.

Mitchison, T.J., and M.W. Kirschner. 1985. Properties of the kinetochore in vitro. II. Microtubule capture and ATP-dependent translocation. J Cell Biol. 101:766-77.

Mitchison, T.J., and E.D. Salmon. 1992. Poleward kinetochore fiber movement occurs during both metaphase and anaphase-A in newt lung cell mitosis. J Cell Biol. 119:569-82.

Mohri, H. 1968. Amino-acid composition of "Tubulin" constituting microtubules of sperm flagella. Nature. 217:1053-4.

Mohri, H., Mohri, T., Mabuchi, I., Yazaki, I., Sakai, H., and Ogawa, K. 1976. Dev. Growth Differ. 18:391.

Mollinari, C., J.-P. Kleman, W. Jiang, G. Schoehn, T. Hunter, and R.L. Margolis. 2002. PRC1 is a microtubule binding and bundling protein essential to maintain the mitotic spindle midzone. J. Cell Biol. 157:1175-1186.

Mollinari, C., C. Reynaud, S. Martineau-Thuillier, S. Monier, S. Kieffer, J. Garin, P.R. Andreassen, A. Boulet, B. Goud, J.P. Kleman, and R.L. Margolis. 2003. The mammalian passenger protein TD-60 is an RCC1 family member with an essential role in prometaphase to metaphase progression. Dev Cell. 5:295-307. 
Moores, C.A., M. Yu, J. Guo, C. Beraud, R. Sakowicz, and R.A. Milligan. 2002. A mechanism for microtubule depolymerization by KinI kinesins. Mol Cell. 9:903-9.

Moritz, M., M.B. Braunfeld, V. Guenebaut, J. Heuser, and D.A. Agard. 2000. Structure of the gamma-tubulin ring complex: a template for microtubule nucleation. Nat Cell Biol. 2:365-70.

Moritz, M., M.B. Braunfeld, J.W. Sedat, B. Alberts, and D.A. Agard. 1995. Microtubule nucleation by gamma-tubulin-containing rings in the centrosome. Nature. 378:638-40.

Morris, N. 2003. Nuclear positioning: the means is at the ends. Current Opinion in Cell Biology. 15:54-59.

Morrison, E.E., B.N. Wardleworth, J.M. Askham, A.F. Markham, and D.M. Meredith. 1998. EB1, a protein which interacts with the APC tumour suppressor, is associated with the microtubule cytoskeleton throughout the cell cycle. Oncogene. 17:3471-7.

Mountain, V., C. Simerly, L. Howard, A. Ando, G. Schatten, and D.A. Compton. 1999. The kinesin-related protein, HSET, opposes the activity of Eg5 and cross-links microtubules in the mammalian mitotic spindle. J Cell Biol. 147:351-66.

Muhua, L., T.S. Karpova, and J.A. Cooper. 1994. A yeast actin-related protein homologous to that in vertebrate dynactin complex is important for spindle orientation and nuclear migration. Cell. 78:669-79.

Muller-Reichert, T., D. Chretien, F. Severin, and A.A. Hyman. 1998. Structural changes at microtubule ends accompanying GTP hydrolysis: information from a slowly hydrolyzable analogue of GTP, guanylyl (alpha,beta)methylenediphosphonate. Proc Natl Acad Sci U S A. 95:3661-6.

Mullins, J.M., and J.R. McIntosh. 1982. Isolation and initial characterization of the mammalian midbody. J Cell Biol. 94:654-61.

Munemitsu, S., B. Souza, O. Muller, I. Albert, B. Rubinfeld, and P. Polakis. 1994. The APC gene product associates with microtubules in vivo and promotes their assembly in vitro. Cancer Res. 54:3676-81.

Murata-Hori, M., M. Tatsuka, and Y.L. Wang. 2002. Probing the dynamics and functions of aurora B kinase in living cells during mitosis and cytokinesis. Mol Biol Cell. 13:1099108.

Murata-Hori, M., and Y. Wang. 2002. The Kinase Activity of Aurora B Is Required for Kinetochore-Microtubule Interactions during Mitosis. Curr Biol. 12:894-9. 
Murphy, D.B., and G.G. Borisy. 1975. Association of high-molecular-weight proteins with microtubules and their role in microtubule assembly in vitro. Proc Natl Acad Sci U S A. 72:2696-700.

Murray, A.W. 1992. Creative blocks: cell-cycle checkpoints and feedback controls. Nature. 359:599-604.

Murray, A.W. 1995. The genetics of cell cycle checkpoints. Curr Opin Genet Dev. 5:5-11.

Nabeshima, K., H. Kurooka, M. Takeuchi, K. Kinoshita, Y. Nakaseko, and M. Yanagida. 1995. p93dis1, which is required for sister chromatid separation, is a novel microtubule and spindle pole body-associating protein phosphorylated at the Cdc2 target sites. Genes Dev. 9:1572-85.

Nabeshima, K., T. Nakagawa, A.F. Straight, A. Murray, Y. Chikashige, Y.M. Yamashita, Y. Hiraoka, and M. Yanagida. 1998. Dynamics of centromeres during metaphaseanaphase transition in fission yeast: Dis1 is implicated in force balance in metaphase bipolar spindle. Mol Biol Cell. 9:3211-25.

Nakamura, M., X.Z. Zhou, and K.P. Lu. 2001. Critical role for the EB1 and APC interaction in the regulation of microtubule polymerization. Curr Biol. 11:1062-7.

Nakaseko, Y., G. Goshima, J. Morishita, and M. Yanagida. 2001. M phase-specific kinetochore proteins in fission yeast: microtubule-associating Dis1 and Mtc1 display rapid separation and segregation during anaphase. Curr Biol. 11:537-49.

Nathke, I.S., C.L. Adams, P. Polakis, J.H. Sellin, and W.J. Nelson. 1996. The adenomatous polyposis coli tumor suppressor protein localizes to plasma membrane sites involved in active cell migration. $J$ Cell Biol. 134:165-79.

Neuwald, A.F., and T. Hirano. 2000. HEAT repeats associated with condensins, cohesins, and other complexes involved in chromosome-related functions. Genome Res. 10:1445-52.

Nguyen, H.L., D. Gruber, and J.C. Bulinski. 1999. Microtubule-associated protein 4 (MAP4) regulates assembly, protomer-polymer partitioning and synthesis of tubulin in cultured cells. J Cell Sci. 112 ( Pt 12):1813-24.

Nicklas, R.B. 1997. How cells get the right chromosomes. Science. 275:632-7.

Nicklas, R.B., M.S. Campbell, S.C. Ward, and G.J. Gorbsky. 1998. Tension-sensitive kinetochore phosphorylation in vitro. J Cell Sci. 111 ( Pt 21):3189-96.

Nicklas, R.B., and C.A. Koch. 1969. Chromosome micromanipulation. 3. Spindle fiber tension and the reorientation of mal-oriented chromosomes. J Cell Biol. 43:40-50. 
Nicklas, R.B., and D.F. Kubai. 1985. Microtubules, chromosome movement, and reorientation after chromosomes are detached from the spindle by micromanipulation. Chromosoma. 92:313-24.

Nicklas, R.B., S.C. Ward, and G.J. Gorbsky. 1995. Kinetochore chemistry is sensitive to tension and may link mitotic forces to a cell cycle checkpoint. J Cell Biol. 130:92939.

Niederstrasser, H., H. Salehi-Had, E.C. Gan, C. Walczak, and E. Nogales. 2002. XKCM1 acts on a single protofilament and requires the C terminus of tubulin. J Mol Biol. 316:81728.

Nislow, C., V.A. Lombillo, R. Kuriyama, and J.R. McIntosh. 1992. A plus-end-directed motor enzyme that moves antiparallel microtubules in vitro localizes to the interzone of mitotic spindles. Nature. 359:543-7.

Nislow, C., C. Sellitto, R. Kuriyama, and J.R. McIntosh. 1990. A monoclonal antibody to a mitotic microtubule-associated protein blocks mitotic progression. J Cell Biol. 111:511-22.

Nogales, E. 2001. Structural insight into microtubule function. Annu Rev Biophys Biomol Struct. 30:397-420.

Nogales, E., M. Whittaker, R.A. Milligan, and K.H. Downing. 1999. High-resolution model of the microtubule. Cell. 96:79-88.

Oakley, C.E., and B.R. Oakley. 1989. Identification of gamma-tubulin, a new member of the tubulin superfamily encoded by mipA gene of Aspergillus nidulans. Nature. 338:6624.

O'Connell, C.B., and Y.L. Wang. 2000. Mammalian spindle orientation and position respond to changes in cell shape in a dynein-dependent fashion. Mol Biol Cell. 11:1765-74.

Ohi, R., M.L. Coughlin, W.S. Lane, and T.J. Mitchison. 2003. An inner centromere protein that stimulates the microtubule depolymerizing activity of a KinI kinesin. Dev Cell. 5:309-21.

Ohkura, H., Y. Adachi, N. Kinoshita, O. Niwa, T. Toda, and M. Yanagida. 1988. Coldsensitive and caffeine-supersensitive mutants of the Schizosaccharomyces pombe dis genes implicated in sister chromatid separation during mitosis. Embo J. 7:1465-73.

Ohkura, H., M.A. Garcia, and T. Toda. 2001. Dis1/TOG universal microtubule adaptors - one MAP for all? J Cell Sci. 114:3805-12. 
Ohkura, H., I.M. Hagan, and D.M. Glover. 1995. The conserved Schizosaccharomyces pombe kinase plo1, required to form a bipolar spindle, the actin ring, and septum, can drive septum formation in G1 and G2 cells. Genes Dev. 9:1059-73.

Olmsted, J.B. 1986. Microtubule-associated proteins. Annu Rev Cell Biol. 2:421-57.

Olmsted, J.B., and G.G. Borisy. 1973. Microtubules. Annu Rev Biochem. 42:507-40.

Olson, K.R., J.R. McIntosh, and J.B. Olmsted. 1995. Analysis of MAP 4 function in living cells using green fluorescent protein (GFP) chimeras. J Cell Biol. 130:639-50.

Ookata, K., S. Hisanaga, J.C. Bulinski, H. Murofushi, H. Aizawa, T.J. Itoh, H. Hotani, E. Okumura, K. Tachibana, and T. Kishimoto. 1995. Cyclin B interaction with microtubule-associated protein 4 (MAP4) targets p34cdc2 kinase to microtubules and is a potential regulator of M-phase microtubule dynamics. J Cell Biol. 128:849-62.

Ookata, K., S. Hisanaga, M. Sugita, A. Okuyama, H. Murofushi, H. Kitazawa, S. Chari, J.C. Bulinski, and T. Kishimoto. 1997. MAP4 is the in vivo substrate for CDC2 kinase in HeLa cells: identification of an M-phase specific and a cell cycle-independent phosphorylation site in MAP4. Biochemistry. 36:15873-83.

Paschal, B.M., H.S. Shpetner, and R.B. Vallee. 1987. MAP 1C is a microtubule-activated ATPase which translocates microtubules in vitro and has dynein-like properties. J Cell Biol. 105:1273-82.

Pasqualone, D., and T.C. Huffaker. 1994. STU1, a suppressor of a beta-tubulin mutation, encodes a novel and essential component of the yeast mitotic spindle. J Cell Biol. 127:1973-84.

Pearson, C.G., P.S. Maddox, T.R. Zarzar, E.D. Salmon, and K. Bloom. 2003. Yeast Kinetochores Do Not Stabilize Stu2p-dependent Spindle Microtubule Dynamics. Mol Biol Cell. 14:4181-95.

Peifer, M., and P. Polakis. 2000. Wnt signaling in oncogenesis and embryogenesis--a look outside the nucleus. Science. 287:1606-9.

Pellman, D., M. Bagget, Y.H. Tu, G.R. Fink, and H. Tu. 1995. Two microtubule-associated proteins required for anaphase spindle movement in Saccharomyces cerevisiae. J Cell Biol. 130:1373-85.

Pereira, A., J. Doshen, E. Tanaka, and L.S. Goldstein. 1992. Genetic analysis of a Drosophila microtubule-associated protein. J Cell Biol. 116:377-83.

Perez, F., G.S. Diamantopoulos, R. Stalder, and T.E. Kreis. 1999. CLIP-170 highlights growing microtubule ends in vivo. Cell. 96:517-27. 
Peters, J.M. 2002. The anaphase-promoting complex: proteolysis in mitosis and beyond. Mol Cell. 9:931-43.

Petersen, J., and I.M. Hagan. 2003. S. pombe Aurora Kinase/Survivin Is Required for Chromosome Condensation and the Spindle Checkpoint Attachment Response. Curr Biol. 13:590-7.

Pfarr, C.M., M. Coue, P.M. Grissom, T.S. Hays, M.E. Porter, and J.R. McIntosh. 1990. Cytoplasmic dynein is localized to kinetochores during mitosis. Nature. 345:263-5.

Pickett-Heaps, J., T. Spurck, and D. Tippit. 1984. Chromosome motion and the spindle matrix. J Cell Biol. 99:137s-143s.

Pickett-Heaps, J.D. 1969. The evolution of the mitotic apparatus. An attemptat comparative ultrastractural cytology in dividing plant cells. Cytobios. 1:257-280.

Pickett-Heaps, J.D., and A. Forer. 2001. Pac-Man does not resolve the enduring problem of anaphase chromosome movement. Protoplasma. 215:16-20.

Pickett-Heaps, J.D., A. Forer, and T. Spurck. 1997. Traction fibre: toward a "tensegral" model of the spindle. Cell Motil Cytoskeleton. 37:1-6.

Piehl, M., and L. Cassimeris. 2003. Organization and Dynamics of Growing Microtubule Plus Ends during Early Mitosis. Mol. Biol. Cell. 14:916-925.

Pierre, P., J. Scheel, J.E. Rickard, and T.E. Kreis. 1992. CLIP-170 links endocytic vesicles to microtubules. Cell. 70:887-900.

Popov, A., F. Severin, and E. Karsenti. 2002. XMAP215 Is Required for the MicrotubuleNucleating Activity of Centrosomes. Curr Biol. 12:1326.

Popov, A.V., A. Pozniakovsky, I. Arnal, C. Antony, A.J. Ashford, K. Kinoshita, R. Tournebize, A.A. Hyman, and E. Karsenti. 2001. XMAP215 regulates microtubule dynamics through two distinct domains. Embo J. 20:397-410.

Powers, J., O. Bossinger, D. Rose, S. Strome, and W. Saxton. 1998. A nematode kinesin required for cleavage furrow advancement. Curr Biol. 8:1133-6.

Putkey, F.R., T. Cramer, M.K. Morphew, A.D. Silk, R.S. Johnson, J.R. McIntosh, and D.W. Cleveland. 2002. Unstable kinetochore-microtubule capture and chromosomal instability following deletion of CENP-E. Dev Cell. 3:351-65.

Qian, Y.W., E. Erikson, C. Li, and J.L. Maller. 1998. Activated polo-like kinase Plx1 is required at multiple points during mitosis in Xenopus laevis. Mol Cell Biol. 18:426271.

Quarmby, L. 2000. Cellular Samurai: katanin and the severing of microtubules. J Cell Sci. 113 ( Pt 16):2821-7. 
Quarmby, L.M., and T.A. Lohret. 1999. Microtubule severing. Cell Motil Cytoskeleton. 43:19.

Quintyne, N.J., S.R. Gill, D.M. Eckley, C.L. Crego, D.A. Compton, and T.A. Schroer. 1999. Dynactin is required for microtubule anchoring at centrosomes. J Cell Biol. 147:32134.

Raff, J.W. 2002. Centrosomes and cancer: lessons from a TACC. Trends Cell Biol. 12:222-5. Raich, W.B., A.N. Moran, J.H. Rothman, and J. Hardin. 1998. Cytokinesis and midzone microtubule organization in Caenorhabditis elegans require the kinesin-like protein ZEN-4. Mol Biol Cell. 9:2037-49.

Rappaport, R. 1961. Experiments concerning the cleavage stimulus in sand dollar eggs. $J$ Exp Zool. 148:81-9.

Rappaport, R. 1986. Establishment of the mechanism of cytokinesis in animal cells. Int Rev Cytol. 105:245-81.

Reed, J.C., and J.R. Bischoff. 2000. BIRinging chromosomes through cell division--and survivin' the experience. Cell. 102:545-8.

Rehberg, M., and R. Graf. 2002. Dictyostelium EB1 Is a Genuine Centrosomal Component Required for Proper Spindle Formation. Mol Biol Cell. 13:2301-10.

Reiner, O., and e. al. 1993. Isolation of a Miller-Dieker lissencephaly gene containing G protein b-subunit-like repeats. Nature. 364:717-721.

Rickard, J.E., and T.E. Kreis. 1990. Identification of a novel nucleotide-sensitive microtubule-binding protein in HeLa cells. J Cell Biol. 110:1623-33.

Rickard, J.E., and T.E. Kreis. 1991. Binding of pp170 to microtubules is regulated by phosphorylation. J Biol Chem. 266:17597-605.

Rieder, C.L. 1982. The formation, structure, and composition of the mammalian kinetochore and kinetochore fiber. Int Rev Cytol. 79:1-58.

Rieder, C.L. 1990. Formation of the astral mitotic spindle: ultrastructural basis for the centrosome-kinetochore interaction. Electron Microsc Rev. 3:269-300.

Rieder, C.L., and S.P. Alexander. 1990. Kinetochores are transported poleward along a single astral microtubule during chromosome attachment to the spindle in newt lung cells. $J$ Cell Biol. 110:81-95.

Rieder, C.L., R.W. Cole, A. Khodjakov, and G. Sluder. 1995. The checkpoint delaying anaphase in response to chromosome monoorientation is mediated by an inhibitory signal produced by unattached kinetochores. J Cell Biol. 130:941-8. 
Rieder, C.L., E.A. Davison, L.C. Jensen, L. Cassimeris, and E.D. Salmon. 1986. Oscillatory movements of monooriented chromosomes and their position relative to the spindle pole result from the ejection properties of the aster and half-spindle. J Cell Biol. 103:581-91.

Rieder, C.L., S. Faruki, and A. Khodjakov. 2001. The centrosome in vertebrates: more than a microtubule-organizing center. Trends Cell Biol. 11:413-9.

Rieder, C.L., A. Khodjakov, L.V. Paliulis, T.M. Fortier, R.W. Cole, and G. Sluder. 1997. Mitosis in vertebrate somatic cells with two spindles: implications for the metaphase/anaphase transition checkpoint and cleavage. Proc Natl Acad Sci U S A. 94:5107-12.

Rieder, C.L., and R.E. Palazzo. 1992. Colcemid and the mitotic cycle. J Cell Sci. 102 ( Pt 3):387-92.

Rieder, C.L., and E.D. Salmon. 1994. Motile kinetochores and polar ejection forces dictate chromosome position on the vertebrate mitotic spindle. J Cell Biol. 124:223-33.

Rieder, C.L., and E.D. Salmon. 1998. The vertebrate cell kinetochore and its roles during mitosis. Trends Cell Biol. 8:310-8.

Rieder, C.L., A. Schultz, R. Cole, and G. Sluder. 1994. Anaphase onset in vertebrate somatic cells is controlled by a checkpoint that monitors sister kinetochore attachment to the spindle. J Cell Biol. 127:1301-10.

Riparbelli, M.G., G. Callaini, D.M. Glover, and C. Avides Md Mdo. 2002. A requirement for the Abnormal Spindle protein to organise microtubules of the central spindle for cytokinesis in Drosophila. J Cell Sci. 115:913-22.

Ris, H., and P.L. Witt. 1981. Structure of the mammalian kinetochore. Chromosoma. 82:15370.

Robinson, J.T., E.J. Wojcik, M.A. Sanders, M. McGrail, and T.S. Hays. 1999. Cytoplasmic dynein is required for the nuclear attachment and migration of centrosomes during mitosis in Drosophila. J Cell Biol. 146:597-608.

Rockmill, B., and S. Fogel. 1988. DIS1: a yeast gene required for proper meiotic chromosome disjunction. Genetics. 119:261-72.

Rodionov, V.I., and G.G. Borisy. 1997. Microtubule treadmilling in vivo. Science. 275:215-8. Rogers, G.C., S.L. Rogers, T.A. Schwimmer, S.C. Ems-McClung, C.E. Walczak, R.D. Vale, J.M. Scholey, and D.J. Sharp. 2004. Two mitotic kinesins cooperate to drive sister chromatid separation during anaphase. Nature. 427:364-70. 
Rogers, S.L., G.C. Rogers, D.J. Sharp, and R.D. Vale. 2002. Drosophila EB1 is important for proper assembly, dynamics, and positioning of the mitotic spindle. J Cell Biol. 158:873-84.

Roghi, C., R. Giet, R. Uzbekov, N. Morin, I. Chartrain, R. Le Guellec, A. Couturier, M. Doree, M. Philippe, and C. Prigent. 1998. The Xenopus protein kinase pEg2 associates with the centrosome in a cell cycle-dependent manner, binds to the spindle microtubules and is involved in bipolar mitotic spindle assembly. J Cell Sci. 111 ( Pt 5):557-72.

Romano, A., A. Guse, I. Krascenicova, H. Schnabel, R. Schnabel, and M. Glotzer. 2003. CSC-1: a subunit of the Aurora B kinase complex that binds to the survivin-like protein BIR-1 and the incenp-like protein ICP-1. J Cell Biol. 161:229-36.

Roof, D.M., P.B. Meluh, and M.D. Rose. 1992. Kinesin-related proteins required for assembly of the mitotic spindle. J Cell Biol. 118:95-108.

Roos, U.P. 1976. Light and electron microscopy of rat kangaroo cells in mitosis. III. Patterns of chromosome behavior during prometaphase. Chromosoma. 54:363-85.

Rubinfeld, B., B. Souza, I. Albert, O. Muller, S.H. Chamberlain, F.R. Masiarz, S. Munemitsu, and P. Polakis. 1993. Association of the APC gene product with beta-catenin. Science. 262:1731-4.

Rudner, A.D., and A.W. Murray. 1996. The spindle assembly checkpoint. Curr Opin Cell Biol. 8:773-80.

Rusan, N.M., C.J. Fagerstrom, A.M. Yvon, and P. Wadsworth. 2001. Cell cycle-dependent changes in microtubule dynamics in living cells expressing green fluorescent proteinalpha tubulin. Mol Biol Cell. 12:971-80.

Sakai, H. 1978. The isolated mitotic apparatus and chromosome motion. Int Rev Cytol. 55:2248.

Sakai, H., Mabushi, I., Shimoda, S., Kuriyama, R., Ogawa, K., and Mohri, H. 1976. Dev. Growth Differ. 18:211.

Salina, D., K. Bodoor, D.M. Eckley, T.A. Schroer, J.B. Rattner, and B. Burke. 2002. Cytoplasmic Dynein as a facilitator of nuclear envelope breakdown. Cell. 108:97-107.

Salmon, E.D. 1988. A model of metaphase chromosome congression and anaphase poleward movement. In Cell movement: kinesin and microtubule-associated protein s. F.D.a.M. Warner, J.R., editor. Alan R. Liss, New York. 431-440.

Salmon, E.D. 1989. Metaphase chromosome congression and anaphase 
poleward movement. In Cell Movement. Vol. 2. F.D. Warner and J.R. MeIntosh, editors. Alan R. Liss, Inc. NY. 431-440.

Sammak, P.J., and G.G. Borisy. 1988. Direct observation of microtubule dynamics in living cells. Nature. 332:724-6.

Sampaio, P., Rebollo, E., Varmark, H., Sunkel, C. E. and Gonzalez, C. 2001. Organized microtubule arrays in gamma-tubulin-depleted Drosophila spermatocytes. Curr. Biol. 11: 1788-93.

Saunders, R.D., M.C. Avides, T. Howard, C. Gonzalez, and D.M. Glover. 1997a. The Drosophila gene abnormal spindle encodes a novel microtubule-associated protein that associates with the polar regions of the mitotic spindle. J Cell Biol. 137:881-90.

Saunders, W., V. Lengyel, and M.A. Hoyt. 1997b. Mitotic spindle function in Saccharomyces cerevisiae requires a balance between different types of kinesin-related motors. $\mathrm{Mol}$ Biol Cell. 8:1025-33.

Saunders, W.S., and M.A. Hoyt. 1992. Kinesin-related proteins required for structural integrity of the mitotic spindle. Cell. 70:451-8.

Savoian, M.S., W.C. Earnshaw, A. Khodjakov, and C.L. Rieder. 1999. Cleavage furrows formed between centrosomes lacking an intervening spindle and chromosomes contain microtubule bundles, INCENP, and CHO1 but not CENP-E. Mol Biol Cell. 10:297-311.

Savoian, M.S., M.L. Goldberg, and C.L. Rieder. 2000. The rate of poleward chromosome motion is attenuated in Drosophila zw10 and rod mutants. Nat Cell Biol. 2:948-52.

Sawin, K.E. 2000. Microtubule dynamics: the view from the tip. Curr Biol. 10:R860-2.

Sawin, K.E., K. LeGuellec, M. Philippe, and T.J. Mitchison. 1992. Mitotic spindle organization by a plus-end-directed microtubule motor. Nature. 359:540-3.

Sawin, K.E., and T.J. Mitchison. 1991a. Mitotic spindle assembly by two different pathways in vitro. J Cell Biol. 112:925-40.

Sawin, K.E., and T.J. Mitchison. 1991b. Poleward microtubule flux mitotic spindles assembled in vitro. J Cell Biol. 112:941-54.

Sawin, K.E., and T.J. Mitchison. 1994. Microtubule flux in mitosis is independent of chromosomes, centrosomes, and antiparallel microtubules. Mol Biol Cell. 5:217-26.

Saxton, W., D. Stemple, R. Leslie, E. Salmon, M. Zavortink, and J. McIntosh. 1984. Tubulin dynamics in cultured mammalian cells. J. Cell Biol. 99:2175-2186.

Schaar, B.T., G.K. Chan, P. Maddox, E.D. Salmon, and T.J. Yen. 1997. CENP-E function at kinetochores is essential for chromosome alignment. J Cell Biol. 139:1373-82. 
Scholey, J.M., B. Neighbors, J.R. McIntosh, and E.D. Salmon. 1984. Isolation of microtubules and a dynein-like MgATPase from unfertilized sea urchin eggs. J Biol Chem. 259:6516-25.

Scholey, J.M., M.E. Porter, P.M. Grissom, and J.R. McIntosh. 1985. Identification of kinesin in sea urchin eggs, and evidence for its localization in the mitotic spindle. Nature. 318:483-6.

Scholey, J.M., G.C. Rogers, and D.J. Sharp. 2001. Mitosis, microtubules, and the matrix. $J$ Cell Biol. 154:261-6.

Schrader, F. 1944. Mitosis - The movements of chromosomes in cell division. Columbia University Press, New York.

Schroeder, T.E. 1972. The contractile ring. II. Determining its brief existence, volumetric changes, and vital role in cleaving Arbacia eggs. J Cell Biol. 53:419-34.

Schroeder, T.E. 1973. Actin in dividing cells: contractile ring filaments bind heavy meromyosin. Proc Natl Acad Sci U S A. 70:1688-92.

Schroer, T.A. 1994. Structure, function and regulation of cytoplasmic dynein. Curr Opin Cell Biol. 6:69-73.

Schulze, E., and M. Kirschner. 1988. New features of microtubule behaviour observed in vivo. Nature. 334:356-9.

Schumacher, J.M., N. Ashcroft, P.J. Donovan, and A. Golden. 1998a. A highly conserved centrosomal kinase, AIR-1, is required for accurate cell cycle progression and segregation of developmental factors in C. elegans embryos. Development. 125:43914402.

Schumacher, J.M., A. Golden, and P.J. Donovan. 1998b. AIR-2: An Aurora/Ipl1-related protein kinase associated with chromosomes and midbody microtubules is required for polar body extrusion and cytokinesis in Caenorhabditis elegans embryos. J Cell Biol. 143:1635-46.

Schuyler, S.C., J.Y. Liu, and D. Pellman. 2003. The molecular function of Ase1p: evidence for a MAP-dependent midzone-specific spindle matrix. Microtubule-associated proteins. J Cell Biol. 160:517-28.

Schuyler, S.C., and D. Pellman. 2001a. Microtubule "plus-end-tracking proteins": The end is just the beginning. Cell. 105:421-4.

Schuyler, S.C., and D. Pellman. 2001b. Search, capture and signal: games microtubules and centrosomes play. J Cell Sci. 114:247-55. 
Schwartz, K., K. Richards, and D. Botstein. 1997. BIM1 encodes a microtubule-binding protein in yeast. Mol Biol Cell. 8:2677-91.

Segerman, B., N. Larsson, P. Holmfeldt, and M. Gullberg. 2000. Mutational analysis of op18/stathmin-tubulin-interacting surfaces. Binding cooperativity controls tubulin GTP hydrolysis in the ternary complex. J Biol Chem. 275:35759-66.

Sellitto, C., and R. Kuriyama. 1988. Distribution of a matrix component of the midbody during the cell cycle in Chinese hamster ovary cells. J Cell Biol. 106:431-9.

Severin, F., B. Habermann, T. Huffaker, and T. Hyman. 2001. Stu2 promotes mitotic spindle elongation in anaphase. J Cell Biol. 153:435-42.

Severin, F.F., P.K. Sorger, and A.A. Hyman. 1997. Kinetochores distinguish GTP from GDP forms of the microtubule lattice. Nature. 388:888-91.

Shah, J.V., and D.W. Cleveland. 2000. Waiting for anaphase: Mad2 and the spindle assembly checkpoint. Cell. 103:997-1000.

Shannon, K.B., J.C. Canman, and E.D. Salmon. 2002. Mad2 and BubR1 function in a single checkpoint pathway that responds to a loss of tension. Mol Biol Cell. 13:3706-19.

Shannon, K.B., and E.D. Salmon. 2002. Chromosome dynamics: new light on aurora B kinase function. Curr Biol. 12:R458-60.

Sharp, D.J. 2002. Cell division: MAST sails through mitosis. Curr Biol. 12:R585-7.

Sharp, D.J., H.M. Brown, M. Kwon, G.C. Rogers, G. Holland, and J.M. Scholey. 2000a. Functional coordination of three mitotic motors in Drosophila embryos. Mol Biol Cell. $11: 241-53$

Sharp, D.J., K.L. McDonald, H.M. Brown, H.J. Matthies, C. Walczak, R.D. Vale, T.J. Mitchison, and J.M. Scholey. 1999a. The bipolar kinesin, KLP61F, cross-links microtubules within interpolar microtubule bundles of Drosophila embryonic mitotic spindles. J Cell Biol. 144:125-38.

Sharp, D.J., G.C. Rogers, and J.M. Scholey. 2000b. Cytoplasmic dynein is required for poleward chromosome movement during mitosis in Drosophila embryos. Nat Cell Biol. 2:922-30.

Sharp, D.J., K.R. Yu, J.C. Sisson, W. Sullivan, and J.M. Scholey. 1999b. Antagonistic microtubule-sliding motors position mitotic centrosomes in Drosophila early embryos. Nat Cell Biol. 1:51-4.

Sharp, L.W. 1934. Introduction to cytology. McGraw-Hill, New York. 
Sharp-Baker, H., and R.H. Chen. 2001. Spindle checkpoint protein Bub1 is required for kinetochore localization of Mad1, Mad2, Bub3, and CENP-E, independently of its kinase activity. J Cell Biol. 153:1239-50.

Shaw, S.L., E. Yeh, P. Maddox, E.D. Salmon, and K. Bloom. 1997. Astral microtubule dynamics in yeast: a microtubule-based searching mechanism for spindle orientation and nuclear migration into the bud. J Cell Biol. 139:985-94.

Sheeman, B., P. Carvalho, I. Sagot, J. Geiser, D. Kho, M.A. Hoyt, and D. Pellman. 2003. Determinants of S. cerevisiae Dynein Localization and Activation. Implications for the Mechanism of Spindle Positioning. Curr Biol. 13:364-72.

Shelanski, M.L., and E.W. Taylor. 1967. Isolation of a protein subunit from microtubules. $J$ Cell Biol. 34:549-54.

Shelden, E., and P. Wadsworth. 1993. Observation and quantification of individual microtubule behavior in vivo: microtubule dynamics are cell-type specific. $J$ Cell Biol. 120:935-45.

Sherline, P., and K. Schiavone. 1978. High molecular weight MAPs are part of the mitotic spindle. J Cell Biol. 77:R9-12.

Shiina, N., T. Moriguchi, K. Ohta, Y. Gotoh, and E. Nishida. 1992. Regulation of a major microtubule-associated protein by MPF and MAP kinase. Embo J. 11:3977-84.

Shiina, N., and S. Tsukita. 1999. Mutations at phosphorylation sites of Xenopus microtubuleassociated protein 4 affect its microtubule-binding ability and chromosome movement during mitosis. Mol Biol Cell. 10:597-608.

Shirasu-Hiza, M., P. Coughlin, and T. Mitchison. 2003. Identification of XMAP215 as a microtubule-destabilizing factor in Xenopus egg extract by biochemical purification. J. Cell Biol. 161:349-358.

Shirayama, M., W. Zachariae, R. Ciosk, and K. Nasmyth. 1998. The Polo-like kinase Cdc5p and the WD-repeat protein Cdc20p/fizzy are regulators and substrates of the anaphase promoting complex in Saccharomyces cerevisiae. Embo J. 17:1336-49.

Shu, H.B., Z. Li, M.J. Palacios, Q. Li, and H.C. Joshi. 1995. A transient association of gamma-tubulin at the midbody is required for the completion of cytokinesis during the mammalian cell division. J Cell Sci. 108 ( Pt 9):2955-62.

Simerly, C., Balczon, R., Brinkley, B. R. and Schatten, G. 1990. Microinjected centromere [corrected] kinetochore antibodies interfere with chromosome movement in meiotic and mitotic mouse oocytes. J. Cell Biol. 111: 1491-504. 
Simon, J.R., and E.D. Salmon. 1990. The structure of microtubule ends during the elongation and shortening phases of dynamic instability examined by negative-stain electron microscopy. J Cell Sci. 96 ( Pt 4):571-82.

Skibbens, R.V., and P. Hieter. 1998. Kinetochores and the checkpoint mechanism that monitors for defects in the chromosome segregation machinery. Annu Rev Genet. 32:307-37.

Skibbens, R.V., V.P. Skeen, and E.D. Salmon. 1993. Directional instability of kinetochore motility during chromosome congression and segregation in mitotic newt lung cells: a push-pull mechanism. J Cell Biol. 122:859-75.

Skop, A.R., H. Liu, J. Yates, B.J. Meyer, and R. Heald. 2002. Functional proteomic analysis of the mammalian midbody reveals conserved cell division components. Mol. Biol. Cell (Supplement). 13:3a

Skoufias, D.A., P.R. Andreassen, F.B. Lacroix, L. Wilson, and R.L. Margolis. 2001. Mammalian mad2 and bub1/bubR1 recognize distinct spindle-attachment and kinetochore-tension checkpoints. Proc Natl Acad Sci U S A. 98:4492-7.

Skoufias, D.A., C. Mollinari, F.B. Lacroix, and R.L. Margolis. 2000. Human survivin is a kinetochore-associated passenger protein. J Cell Biol. 151:1575-82.

Sluder, G. 1990. Functional properties of kinetochores in animal cells. Curr Opin Cell Biol. 2:23-7.

Sluder, G., F.J. Miller, E.A. Thompson, and D.E. Wolf. 1994. Feedback control of the metaphase-anaphase transition in sea urchin zygotes: role of maloriented chromosomes. J Cell Biol. 126:189-98.

Smirnova, E.A., and A.S. Bajer. 1992. Spindle poles in higher plant mitosis. Cell Motil Cytoskeleton. 23:1-7.

Solomon, F., M. Magendantz, and A. Salzman. 1979. Identification with cellular microtubules of one of the co-assemlbing microtubule-associated proteins. Cell. 18:431-8.

Somma, M.P., B. Fasulo, G. Cenci, E. Cundari, and M. Gatti. 2002. Molecular dissection of cytokinesis by RNA interference in Drosophila cultured cells. Mol Biol Cell. 13:244860.

Speliotes, E.K., Uren, A., Vaux, D. and Horvitz, H.R. 2000. The survivin-like C. elegans BIR-1 protein acts with the aurora-like kinase AIR-2 to affect chromosomes and the spindle midzone. Mol Cell. 6:211-223. 
Spittle, C., S. Charrasse, C. Larroque, and L. Cassimeris. 2000. The interaction of TOGp with microtubules and tubulin. J Biol Chem. 275:20748-53.

Srayko, M., D.W. Buster, O.A. Bazirgan, F.J. McNally, and P.E. Mains. 2000. MEI-1/MEI-2 katanin-like microtubule severing activity is required for Caenorhabditis elegans meiosis. Genes Dev. 14:1072-84.

Srayko, M., S. Quintin, A. Schwager, and A.A. Hyman. 2003. Caenorhabditis elegans TAC-1 and ZYG-9 form a complex that is essential for long astral and spindle microtubules. Curr Biol. 13:1506-11.

Starr, D.A., B.C. Williams, T.S. Hays, and M.L. Goldberg. 1998. ZW10 helps recruit dynactin and dynein to the kinetochore. J Cell Biol. 142:763-74.

Stearns, T. 1997. Motoring to the Finish: Kinesin and Dynein Work Together to Orient the Yeast Mitotic Spindle. J. Cell Biol. 138:957-960.

Steinmetz, M.O., R.A. Kammerer, W. Jahnke, K.N. Goldie, A. Lustig, and J. van Oostrum. 2000. Op18/stathmin caps a kinked protofilament-like tubulin tetramer. Embo J. 19:572-80.

Stern, B.M. 2002. Mitosis: aurora gives chromosomes a healthy stretch. Curr Biol. 12:R3168.

Steuer, E.R., L. Wordeman, T.A. Schroer, and M.P. Sheetz. 1990. Localization of cytoplasmic dynein to mitotic spindles and kinetochores. Nature. 345:266-8.

Stoppin-Mellet, V., J. Gaillard, and M. Vantard. 2002. Functional evidence for in vitro microtubule severing by the plant katanin homologue. Biochem J. 365:337-42.

Strahler, J.R., B.J. Lamb, D.R. Ungar, D.A. Fox, and S.M. Hanash. 1992. Cell cycle progression is associated with distinct patterns of phosphorylation of Op18. Biochem Biophys Res Commun. 185:197-203.

Straight, A.F., and C.M. Field. 2000. Microtubules, membranes and cytokinesis. Curr Biol. 10:R760-70.

Stucke, V.M., H.H. Sillje, L. Arnaud, and E.A. Nigg. 2002. Human Mps1 kinase is required for the spindle assembly checkpoint but not for centrosome duplication. Embo J. 21:1723-32.

Su, L.K., M. Burrell, D.E. Hill, J. Gyuris, R. Brent, R. Wiltshire, J. Trent, B. Vogelstein, and K.W. Kinzler. 1995. APC binds to the novel protein EB1. Cancer Res. 55:2972-7.

Su, L.K., B. Vogelstein, and K.W. Kinzler. 1993. Association of the APC tumor suppressor protein with catenins. Science. 262:1734-7. 
Sunkel, C.E., and D.M. Glover. 1988. polo, a mitotic mutant of Drosophila displaying abnormal spindle poles. Journal of Cell Science. 89:25-38.

Tai, C.-Y., D.L. Dujardin, N.E. Faulkner, and R.B. Vallee. 2002. Role of dynein, dynactin, and CLIP-170 interactions in LIS1 kinetochore function. J. Cell Biol. 0.

Tanaka, T.U., N. Rachidi, C. Janke, G. Pereira, M. Galova, E. Schiebel, M.J. Stark, and K. Nasmyth. 2002. Evidence that the Ipl1-Sli15 (Aurora kinase-INCENP) complex promotes chromosome bi-orientation by altering kinetochore-spindle pole connections. Cell. 108:317-29.

Tang, Z., R. Bharadwaj, B. Li, and H. Yu. 2001. Mad2-Independent inhibition of APCCdc20 by the mitotic checkpoint protein BubR1. Dev Cell. 1:227-37.

Tavares, A.A., D.M. Glover, and C.E. Sunkel. 1996. The conserved mitotic kinase polo is regulated by phosphorylation and has preferred microtubule-associated substrates in Drosophila embryo extracts. Embo J. 15:4873-83.

Taylor, S.S., E. Ha, and F. McKeon. 1998. The human homologue of Bub3 is required for kinetochore localization of Bub1 and a Mad3/Bub1-related protein kinase. J Cell Biol. 142:1-11.

Taylor, S.S., and F. McKeon. 1997. Kinetochore localization of murine Bub1 is required for normal mitotic timing and checkpoint response to spindle damage. Cell. 89:727-35.

ten Hoopen, R., T. Schleker, R. Manteuffel, and I. Schubert. 2002. Transient CENP-E-like kinetochore proteins in plants. Chromosome Res. 10:561-70.

Terada, Y., M. Tatsuka, F. Suzuki, Y. Yasuda, S. Fujita, and M. Otsu. 1998. AIM-1: a mammalian midbody-associated protein required for cytokinesis. Embo J. 17:667-76.

Theesfeld, C.L., J.E. Irazoqui, K. Bloom, and D.J. Lew. 1999. The role of actin in spindle orientation changes during the Saccharomyces cerevisiae cell cycle. J Cell Biol. 146:1019-32.

Thrower, D.A., M.A. Jordan, B.T. Schaar, T.J. Yen, and L. Wilson. 1995. Mitotic HeLa cells contain a CENP-E-associated minus end-directed microtubule motor. Embo J. 14:91826.

Tilney, L.G., J. Bryan, D.J. Bush, K. Fujiwara, M.S. Mooseker, D.B. Murphy, and D.H. Snyder. 1973. Microtubules: evidence for 13 protofilaments. J Cell Biol. 59:267-75.

Tippit, D.H., J.D. Pickett-Heaps, and R. Leslie. 1980. Cell division in two large pennate diatoms Hantzschia and Nitzschia III. A new proposal for kinetochore function during prometaphase. J Cell Biol. 86:402-16. 
Tirnauer, J.S., and B.E. Bierer. 2000. EB1 proteins regulate microtubule dynamics, cell polarity, and chromosome stability. J Cell Biol. 149:761-6.

Tirnauer, J.S., J.C. Canman, E.D. Salmon, and T.J. Mitchison. 2002a. EB1 Targets to Kinetochores with Attached, Polymerizing Microtubules. Mol. Biol. Cell. 13:43084316.

Tirnauer, J.S., S. Grego, E.D. Salmon, and T.J. Mitchison. 2002b. EB1-Microtubule Interactions in Xenopus Egg Extracts: Role of EB1 in Microtubule Stabilization and Mechanisms of Targeting to Microtubules. Mol. Biol. Cell. 13:3614-3626.

Tirnauer, J.S., E. O'Toole, L. Berrueta, B.E. Bierer, and D. Pellman. 1999. Yeast Bim1p Promotes the G1-specific Dynamics of Microtubules. J. Cell Biol. 145:993-1007.

Tokai, N., A. Fujimoto-Nishiyama, Y. Toyoshima, S. Yonemura, S. Tsukita, J. Inoue, and T. Yamamota. 1996. Kid, a novel kinesin-like DNA binding protein, is localized to chromosomes and the mitotic spindle. Embo J. 15:457-67.

Tombes, R.M., J.G. Peloquin, and G.G. Borisy. 1991. Specific association of an M-phase kinase with isolated mitotic spindles and identification of two of its substrates as MAP4 and MAP1B. Cell Regul. 2:861-74.

Tournebize, R., S.S. Andersen, F. Verde, M. Doree, E. Karsenti, and A.A. Hyman. 1997. Distinct roles of PP1 and PP2A-like phosphatases in control of microtubule dynamics during mitosis. Embo J. 16:5537-49.

Tournebize, R., A. Popov, K. Kinoshita, A.J. Ashford, S. Rybina, A. Pozniakovsky, T.U. Mayer, C.E. Walczak, E. Karsenti, and A.A. Hyman. 2000. Control of microtubule dynamics by the antagonistic activities of XMAP215 and XKCM1 in Xenopus egg extracts. Nat Cell Biol. 2:13-9.

Tran, P.T., R.A. Walker, and E.D. Salmon. 1997. A metastable intermediate state of microtubule dynamic instability that differs significantly between plus and minus ends. J Cell Biol. 138:105-17.

Trinkle-Mulcahy, L., P.D. Andrews, S. Wickramasinghe, J. Sleeman, A. Prescott, Y.W. Lam, C. Lyon, J.R. Swedlow, and A.I. Lamond. 2003. Time-lapse imaging reveals dynamic relocalization of PP1gamma throughout the mammalian cell cycle. Mol Biol Cell. 14:107-17.

Trzepacz, C., A.M. Lowy, J.J. Kordich, and J. Groden. 1997. Phosphorylation of the tumor suppressor adenomatous polyposis coli (APC) by the cyclin-dependent kinase p34. $J$ Biol Chem. 272:21681-4. 
Tsai, M.Y., C. Wiese, K. Cao, O. Martin, P. Donovan, J. Ruderman, C. Prigent, and Y. Zheng. 2003. A Ran signalling pathway mediated by the mitotic kinase Aurora A in spindle assembly. Nat Cell Biol. 5:242-8.

Uren, A.G., T. Beilharz, M.J. O’Connell, S.J. Bugg, R. van Driel, D.L. Vaux, and L. T. 1999. Role for yeast inhibitor of apoptosis IAP-like proteins in

cell division. Proc Natl Acad Sci USA. 96:10170-10175.

Uren, A.G., L. Wong, M. Pakusch, K.J. Fowler, F.J. Burrows, D.L. Vaux, and K.H. Choo. 2000. Survivin and the inner centromere protein INCENP show similar cell-cycle localization and gene knockout phenotype. Curr Biol. 10:1319-28.

Vaisberg, E.A., M.P. Koonce, and J.R. McIntosh. 1993. Cytoplasmic dynein plays a role in mammalian mitotic spindle formation. J Cell Biol. 123:849-58.

Vale, R.D. 1991. Severing of stable microtubules by a mitotically activated protein in Xenopus egg extracts. Cell. 64:827-39.

Vale, R.D., and R.J. Fletterick. 1997. The design plan of kinesin motors. Annu Rev Cell Dev Biol. 13:745-777.

Vale, R.D., F. Malik, and D. Brown. 1992. Directional instability of microtubule transport in the presence of kinesin and dynein, two opposite polarity motor proteins. J Cell Biol. 119:1589-96.

Vale, R.D., T.S. Reese, and M.P. Sheetz. 1985. Identification of a novel force-generating protein, kinesin, involved in microtubule-based motility. Cell. 42:39-50.

Valetti, C., D.M. Wetzel, M. Schrader, M.J. Hasbani, S.R. Gill, T.E. Kreis, and T.A. Schroer. 1999. Role of dynactin in endocytic traffic: effects of dynamitin overexpression and colocalization with CLIP-170. Mol Biol Cell. 10:4107-20.

Vallee, R.B., and G.S. Bloom. 1983. Isolation of sea urchin egg microtubules with taxol and identification of mitotic spindle microtubule-associated proteins with monoclonal antibodies. Proc Natl Acad Sci U S A. 80:6259-63.

Vallee, R.B., and M.A. Gee. 1998. Make room for dynein. Trends Cell Biol. 8:490-4.

van Breugel, M., D. Drechsel, and A. Hyman. 2003. Stu2p, the budding yeast member of the conserved Dis1/XMAP215 family of microtubule-associated proteins is a plus endbinding microtubule destabilizer. J. Cell Biol. 161:359-369.

Van Hooser, A.A., and R. Heald. 2001. Kinetochore function: the complications of becoming attached. Curr Biol. 11:R855-7.

Vanderslice, R., and D. Hirsh. 1976. Temperature-sensitive zygote defective mutants of Caenorhabditis elegans. Dev Biol. 49:236-49. 
Vandre, D.D., V.E. Centonze, J. Peloquin, R.M. Tombes, and G.G. Borisy. 1991. Proteins of the mammalian mitotic spindle: phosphorylation/dephosphorylation of MAP-4 during mitosis. J Cell Sci. 98 ( Pt 4):577-88.

Vasquez, R., D. Gard, and L. Cassimeris. 1994. XMAP from Xenopus eggs promotes rapid plus end assembly of microtubules and rapid microtubule polymer turnover. J. Cell Biol. 127:985-993.

Vasquez, R.J., D.L. Gard, and L. Cassimeris. 1999. Phosphorylation by CDK1 regulates XMAP215 function in vitro. Cell Motil Cytoskeleton. 43:310-21.

Vaughan, K.T., S.H. Tynan, N.E. Faulkner, C.J. Echeverri, and R.B. Vallee. 1999. Colocalization of cytoplasmic dynein with dynactin and CLIP-170 at microtubule distal ends. J Cell Sci. 112 ( Pt 10):1437-47.

Verde, F., J. Labbé, M. Dorée, and E. Karsenti. 1990. Regulation of microtubule dynamics by cdc2 protein kinase in cell-free extracts of Xenopus eggs. Nature. 343:233-238.

Vernos, I., and E. Karsenti. 1996. Motors involved in spindle assembly and chromosome segregation. Curr Opin Cell Biol. 8:4-9.

Vernos, I., J. Raats, T. Hirano, J. Heasman, E. Karsenti, and C. Wylie. 1995. Xklp1, a chromosomal Xenopus kinesin-like protein essential for spindle organization and chromosome positioning. Cell. 81:117-27.

Wade, R.H., and A.A. Hyman. 1997. Microtubule structure and dynamics. Curr Opin Cell Biol. 9:12-7.

Wakefield, J.G., S. Bonaccorsi, and M. Gatti. 2001. The drosophila protein asp is involved in microtubule organization during spindle formation and cytokinesis. J Cell Biol. 153:637-48.

Walczak, C.E. 2000. Microtubule dynamics and tubulin interacting proteins. Curr Opin Cell Biol. 12:52-6.

Walczak, C.E. 2003. The Kin I kinesins are microtubule end-stimulated ATPases. Mol Cell. 11:286-8.

Walczak, C.E., E.C. Gan, A. Desai, T.J. Mitchison, and S.L. Kline-Smith. 2002. The Microtubule-Destabilizing Kinesin XKCM1 Is Required for Chromosome Positioning during Spindle Assembly. Curr Biol. 12:1885-9.

Walczak, C.E., T.J. Mitchison, and A. Desai. 1996. XKCM1: a Xenopus kinesin-related protein that regulates microtubule dynamics during mitotic spindle assembly. Cell. 84:37-47. 
Walczak, C.E., S. Verma, and T.J. Mitchison. 1997. XCTK2: a kinesin-related protein that promotes mitotic spindle assembly in Xenopus laevis egg extracts. $J$ Cell Biol. 136:859-70.

Walczak, C.E., I. Vernos, T.J. Mitchison, E. Karsenti, and R. Heald. 1998. A model for the proposed roles of different microtubule-based motor proteins in establishing spindle bipolarity. Curr Biol. 8:903-13.

Walker, R.A., E.T. O'Brien, N.K. Pryer, M.F. Soboeiro, W.A. Voter, H.P. Erickson, and E.D. Salmon. 1988. Dynamic instability of individual microtubules analyzed by video light microscopy: rate constants and transition frequencies. J Cell Biol. 107:1437-48.

Walker, R.A., N.K. Pryer, and E.D. Salmon. 1991. Dilution of individual microtubules observed in real time in vitro: evidence that cap size is small and independent of elongation rate. J Cell Biol. 114:73-81.

Wang, P.J., and T.C. Huffaker. 1997. Stu2p: A microtubule-binding protein that is an essential component of the yeast spindle pole body. J Cell Biol. 139:1271-80.

Wang, S., and R. Adler. 1995. Chromokinesin: a DNA-binding, kinesin-like nuclear protein. J. Cell Biol. 128:761-768.

Wang, X.M., J.G. Peloquin, Y. Zhai, J.C. Bulinski, and G.G. Borisy. 1996. Removal of MAP4 from microtubules in vivo produces no observable phenotype at the cellular level. J Cell Biol. 132:345-57.

Wang, Y., and D.J. Burke. 1997. Cdc55p, the B-type regulatory subunit of protein phosphatase 2A, has multiple functions in mitosis and is required for the kinetochore/spindle checkpoint in Saccharomyces cerevisiae. Mol Cell Biol. 17:620-6.

Wang, Y.L. 2001. The mechanism of cytokinesis: reconsideration and reconciliation. Cell Struct Funct. 26:633-8.

Waterman-Storer, C.M., and E.D. Salmon. 1997. Microtubule dynamics: treadmilling comes around again. Curr Biol. 7:R369-72.

Waters, J.C., R.H. Chen, A.W. Murray, G.J. Gorbsky, E.D. Salmon, and R.B. Nicklas. 1999. Mad2 binding by phosphorylated kinetochores links error detection and checkpoint action in mitosis. Curr Biol. 9:649-52.

Waters, J.C., R.H. Chen, A.W. Murray, and E.D. Salmon. 1998. Localization of Mad2 to kinetochores depends on microtubule attachment, not tension. J Cell Biol. 141:118191. 
Waters, J.C., R.W. Cole, and C.L. Rieder. 1993. The force-producing mechanism for centrosome separation during spindle formation in vertebrates is intrinsic to each aster. J Cell Biol. 122:361-72.

Waters, J.C., T.J. Mitchison, C.L. Rieder, and E.D. Salmon. 1996. The kinetochore microtubule minus-end disassembly associated with poleward flux produces a force that can do work. Mol Biol Cell. 7:1547-58.

Weisenberg, R.C. 1972. Microtubule formation in vitro in solutions containing low calcium concentrations. Science. 177:1104-5.

Weisenberg, R.C., and Taylor, E.W. 1968. Studies on the ATPase activity of sea urchin eggs and the isolated mitotic apparatus. Exp. Cell Res. 53:372-384.

Weisenberg, R.C., G.G. Borisy, and E.W. Taylor. 1968. The colchicine-binding protein of mammalian brain and its relation to microtubules. Biochemistry. 7:4466-79.

Weisenberg, R.C., W.J. Deery, and P.J. Dickinson. 1976. Tubulin-nucleotide interactions during the polymerization and depolymerization of microtubules. Biochemistry. 15:4248-54.

Weiss, E., and M. Winey. 1996. The Saccharomyces cerevisiae spindle pole body duplication gene MPS1 is part of a mitotic checkpoint. J Cell Biol. 132:111-23.

Wells, W.A. 2001. Searching for a spindle matrix. J Cell Biol. 154:1102-4.

Wheatley, S.P., A. Carvalho, P. Vagnarelli, and W.C. Earnshaw. 2001a. INCENP is required for proper targeting of Survivin to the centromeres and the anaphase spindle during mitosis. Curr Biol. 11:886-90.

Wheatley, S.P., E.H. Hinchcliffe, M. Glotzer, A.A. Hyman, G. Sluder, and Y. Wang. 1997. CDK1 inactivation regulates anaphase spindle dynamics and cytokinesis in vivo. $J$ Cell Biol. 138:385-93.

Wheatley, S.P., S.E. Kandels-Lewis, R.R. Adams, A.M. Ainsztein, and W.C. Earnshaw. 2001b. INCENP binds directly to tubulin and requires dynamic microtubules to target to the cleavage furrow. Exp Cell Res. 262:122-7.

Wheatley, S.P., C.B. O'Connell, and Y. Wang. 1998. Inhibition of chromosomal separation provides insights into cleavage furrow stimulation in cultured epithelial cells. Mol Biol Cell. 9:2173-84.

Wheatley, S.P., and Y. Wang. 1996. Midzone microtubule bundles are continuously required for cytokinesis in cultured epithelial cells. J Cell Biol. 135:981-9. 
Whittington, A.T., O. Vugrek, K.J. Wei, N.G. Hasenbein, K. Sugimoto, M.C. Rashbrooke, and G.O. Wasteneys. 2001. MOR1 is essential for organizing cortical microtubules in plants. Nature. 411:610-3.

Wiche, G., V.G. Corces, and J. Avila. 1978. Preferential binding of hog brain microtubuleassociated proteins to mouse satellite versus bulk DNA preparations. Nature. 273:4035.

Wiese, C., and Y. Zheng. 2000. A new function for the gamma-tubulin ring complex as a microtubule minus-end cap. Nat Cell Biol. 2:358-64.

Williams, B.C., M. Gatti, and M.L. Goldberg. 1996. Bipolar spindle attachments affect redistributions of ZW10, a Drosophila centromere/kinetochore component required for accurate chromosome segregation. J Cell Biol. 134:1127-40.

Williams, B.C., M.F. Riedy, E.V. Williams, M. Gatti, and M.L. Goldberg. 1995. The Drosophila kinesin-like protein KLP3A is a midbody component required for central spindle assembly and initiation of cytokinesis. J Cell Biol. 129:709-23.

Wilson, E.B. 1925. The Cell in Development and Heredity. The Macmillan Company, $3^{\text {rd }}$ edition, New York.

Winey, M., and B. Byers. 1993. Assembly and functions of the spindle pole body in budding yeast. Trends Genet. 9:300-4.

Winey, M., L. Goetsch, P. Baum, and B. Byers. 1991. MPS1 and MPS2: novel yeast genes defining distinct steps of spindle pole body duplication. J Cell Biol. 114:745-54.

Witt, P.L., H. Ris, and G.G. Borisy. 1981. Structure of kinetochore fibers: microtubule continuity and inter-microtubule bridges. Chromosoma. 83:523-40.

Wittmann, T., H. Boleti, C. Antony, E. Karsenti, and I. Vernos. 1998. Localization of the kinesin-like protein Xklp2 to spindle poles requires a leucine zipper, a microtubuleassociated protein, and dynein. J Cell Biol. 143:673-85.

Wittmann, T., A. Hyman, and A. Desai. 2001. The spindle: a dynamic assembly of microtubules and motors. Nat Cell Biol. 3:E28-34.

Wittmann, T., M. Wilm, E. Karsenti, and I. Vernos. 2000. TPX2, A novel xenopus MAP involved in spindle pole organization. J Cell Biol. 149:1405-18.

Wojcik, E., R. Basto, M. Serr, F. Scaerou, R. Karess, and T. Hays. 2001. Kinetochore dynein: its dynamics and role in the transport of the Rough deal checkpoint protein. Nat Cell Biol. 3:1001-7. 
Wood, K.W., R. Sakowicz, L.S. Goldstein, and D.W. Cleveland. 1997. CENP-E is a plus end-directed kinetochore motor required for metaphase chromosome alignment. Cell. 91:357-66.

Wood, W.B., R. Hecht, S. Carr, R. Vanderslice, N. Wolf, and D. Hirsh. 1980. Parental effects and phenotypic characterization of mutations that affect early development in Caenorhabditis elegans. Dev Biol. 74:446-69.

Wordeman, L., and T.J. Mitchison. 1995. Identification and partial characterization of mitotic centromere-associated kinesin, a kinesin-related protein that associates with centromeres during mitosis. J Cell Biol. 128:95-104.

Wordeman, L., E.R. Steuer, M.P. Sheetz, and T. Mitchison. 1991. Chemical subdomains within the kinetochore domain of isolated CHO mitotic chromosomes. J Cell Biol. 114:285-94.

Yajima, J., M. Edamatsu, J. Watai-Nishii, N. Tokai-Nishizumi, T. Yamamoto, and Y.Y. Toyoshima. 2003. The human chromokinesin Kid is a plus end-directed microtubulebased motor. EMBO J. 22:1067-1074.

Yang, C.H., and M. Snyder. 1992. The nuclear-mitotic apparatus protein is important in the establishment and maintenance of the bipolar mitotic spindle apparatus. Mol Biol Cell. 3:1259-67.

Yao, X., K.L. Anderson, and D.W. Cleveland. 1997. The microtubule-dependent motor centromere-associated protein E (CENP-E) is an integral component of kinetochore corona fibers that link centromeres to spindle microtubules. J Cell Biol. 139:435-47.

Yeh, E., R.V. Skibbens, J.W. Cheng, E.D. Salmon, and K. Bloom. 1995. Spindle dynamics and cell cycle regulation of dynein in the budding yeast, Saccharomyces cerevisiae. $J$ Cell Biol. 130:687-700.

Yeh, E., C. Yang, E. Chin, P. Maddox, E.D. Salmon, D.J. Lew, and K. Bloom. 2000. Dynamic positioning of mitotic spindles in yeast: role of microtubule motors and cortical determinants. Mol Biol Cell. 11:3949-61.

Yen, T.J., D.A. Compton, D. Wise, R.P. Zinkowski, B.R. Brinkley, W.C. Earnshaw, and D.W. Cleveland. 1991. CENP-E, a novel human centromere-associated protein required for progression from metaphase to anaphase. Embo J. 10:1245-54.

Yen, T.J., G. Li, B.T. Schaar, I. Szilak, and D.W. Cleveland. 1992. CENP-E is a putative kinetochore motor that accumulates just before mitosis. Nature. 359:536-9.

Yin, H., D. Pruyne, T.C. Huffaker, and A. Bretscher. 2000. Myosin V orientates the mitotic spindle in yeast. Nature. 406:1013-5. 
Yin, H., L. You, D. Pasqualone, K.M. Kopski, and T.C. Huffaker. 2002. Stu1p Is Physically Associated with beta-Tubulin and Is Required for Structural Integrity of the Mitotic Spindle. Mol Biol Cell. 13:1881-92.

Yoon, H.J., and J. Carbon. 1999. Participation of Bir1p, a member of the inhibitor of apoptosis family, in yeast chromosome segregation events. Proc Natl Acad Sci USA. 96:13208-13213.

Yoshida, T., A. Ito, and K. Izutsu. 1985. Association of anti-dynein-1 cross-reactive antigen with the mitotic spindle of mammalian cells. Cell Struct Funct. 10:245-58.

Yu, X., L. Waltzer, and M. Bienz. 1999. A new Drosophila APC homologue associated with adhesive zones of epithelial cells. Nature Cell Biol. 1:144-151.

Yucel, J.K., J.D. Marszalek, J.R. McIntosh, L.S. Goldstein, D.W. Cleveland, and A.V. Philp. 2000. CENP-meta, an essential kinetochore kinesin required for the maintenance of metaphase chromosome alignment in Drosophila. J Cell Biol. 150:1-11.

Zachariae, W., and K. Nasmyth. 1999. Whose end is destruction: cell division and the anaphase-promoting complex. Genes Dev. 13:2039-58.

Zeitlin, S.G., and K.F. Sullivan. 2001. Animal cytokinesis: breaking up is hard to do. Curr Biol. 11:R514-6.

Zhai, Y., P. Kronebusch, P. Simon, and G. Borisy. 1996. Microtubule dynamics at the G2/M transition: abrupt breakdown of cytoplasmic microtubules at nuclear envelope breakdown and implications for spindle morphogenesis. J. Cell Biol. 135:201-214.

Zhai, Y., P.J. Kronebusch, and G.G. Borisy. 1995. Kinetochore microtubule dynamics and the metaphase-anaphase transition. J Cell Biol. 131:721-34.

Zhang, D., and R.B. Nicklas. 1996. 'Anaphase' and cytokinesis in the absence of chromosomes. Nature. 382:466-8.

Zheng, Y., M.L. Wong, B. Alberts, and T. Mitchison. 1995. Nucleation of microtubule assembly by a gamma-tubulin-containing ring complex. Nature. 378:578-83.

Zieve, G.a.S., F. 1982. Proteins specifically associated with the microtubules of the mammalian mitotic spindle. Cell. 28:233-242.

Zinkowski, R.P., J. Meyne, and B.R. Brinkley. 1991. The centromere-kinetochore complex: a repeat subunit model. J Cell Biol. 113:1091-110.

Zirkle, R.E. 1970. Ultraviolet-microbeam irradiation of newt-cell cytoplasm: spindle destruction, false anaphase, and delay of true anaphase. Radiat Res. 41:516-37. 
Zumbrunn, J., K. Kinoshita, A.A. Hyman, and I.S. Nathke. 2001. Binding of the adenomatous polyposis coli protein to microtubules increases microtubule stability and is regulated by GSK3 beta phosphorylation. Curr Biol. 11:44-9. 


\section{Figure legends}

Figure 1. Different mitotic stages in Xenopus A6 culture cells. Only DNA is shown. (A) Prophase; (B) Prometaphase; (C) Metaphase; (D) Anaphase A; (E) Anaphase B; (F) Telophase/. Bar is $10 \mu \mathrm{m}$.

Figure 2. Spindle organization at metaphase. Visualization of spindle microtubules in a live Drosophila S2 tissue culture cell stably transfected with a construct that expresses GFPtubulin. Note the microtubule bundles that extend from the poles to the center of the cell and the many astral microtubules nucleated by the spindle poles. Bar is $10 \mu \mathrm{m}$.

Figure 3. Time-lapse images of spindle formation in a Drosophila S2 tissue culture cell stably expressing GFP-tubulin. A) Prophase, note that the nucleus is clear of microtubules. B) Very early prometaphase indicated by nuclear envelope breakdown and GFP signal within the nucleus. C) Mid- prometaphase as the microtubules begin to set up the spindle. D) Late

prometaphase. E) Spindle organization at metaphase. Note the robust microtubule bundles. F) Anaphase A, when sister chromatids come apart but the pole-to-pole distance has not elongated. G) and H) Anaphase A and anaphase B showing the significant elongation of the spindle. I) Telophase as indicated by the reformation of the two daughter nuclei. Bar is 10 $\mu \mathrm{m}$.

Figure 4. Localization of MAPs to multiple mitotic compartments in human HeLa cells. Microtubules are in red, DNA in blue and CLASP1 in green. (A) Prophase - CLASP1 is mainly at the centrosomes. (B) Prometaphase - In addition to centrosomes, CLASP1 starts accumulating at kinetochores. (C) Metaphase - CLASP1 localizes at centrosomes and 
kinetochores of bioriented chromosomes. (D, E) Anaphase A and B - CLASP1 redistributes to the spindle mid-zone. (F) Telophase/Cytokinesis - CLASP1 accumulates at the mid-body matrix as two parallel marks. Bar is $10 \mu \mathrm{m}$.

Figure 5. Loss of spindle bipolarity after depletion of MAST in Drosophila. In all the images DNA is shown in blue, tubulin in green and centrosomes in yellow. A) Wild type neuroblast showing the normal organization of the metaphase spindle. B) and C) mutant mast ${ }^{1}$ neuroblasts in which the spindle shows a monopolar organization with that chromosomes localized closed to the centrosome.

Figure 6. Schematic representation of the spindle during metaphase and anaphase. The different localization patterns of the various MAPs is indicated (see also Table 1). (1) Microtubule plus-end-binding proteins; (2) Centrosome or pole-associated proteins; (3) Chromosomal or kinetochore proteins; (4) Centrosome or pole-associated proteins that remain during anaphase; (5) Chromosomal or kinetochore proteins that remain during anaphase; (6) Proteins associated with the spindle mid-zone during anaphase and cytokinesis. Note that Aurora A and B have not been shown to bind microtubules directly. 


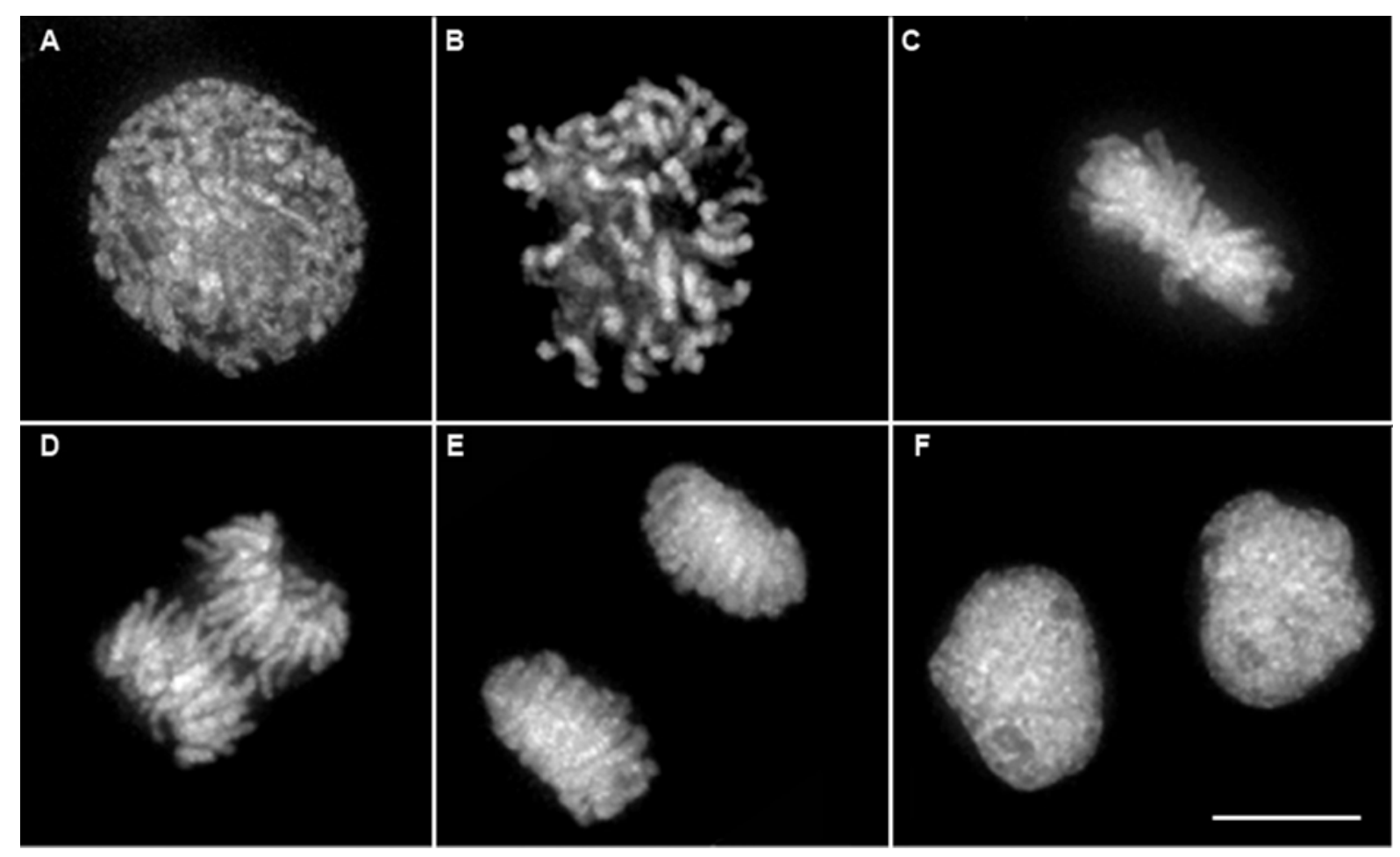

FIGURE 1 


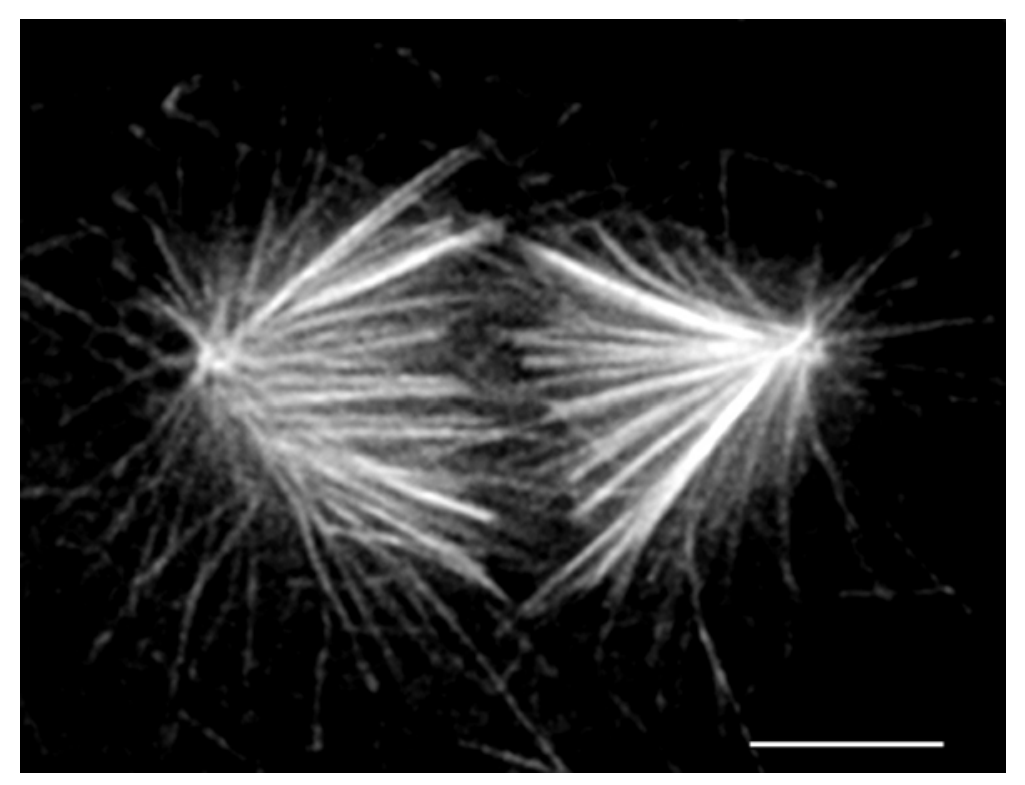

FIGURE 2 


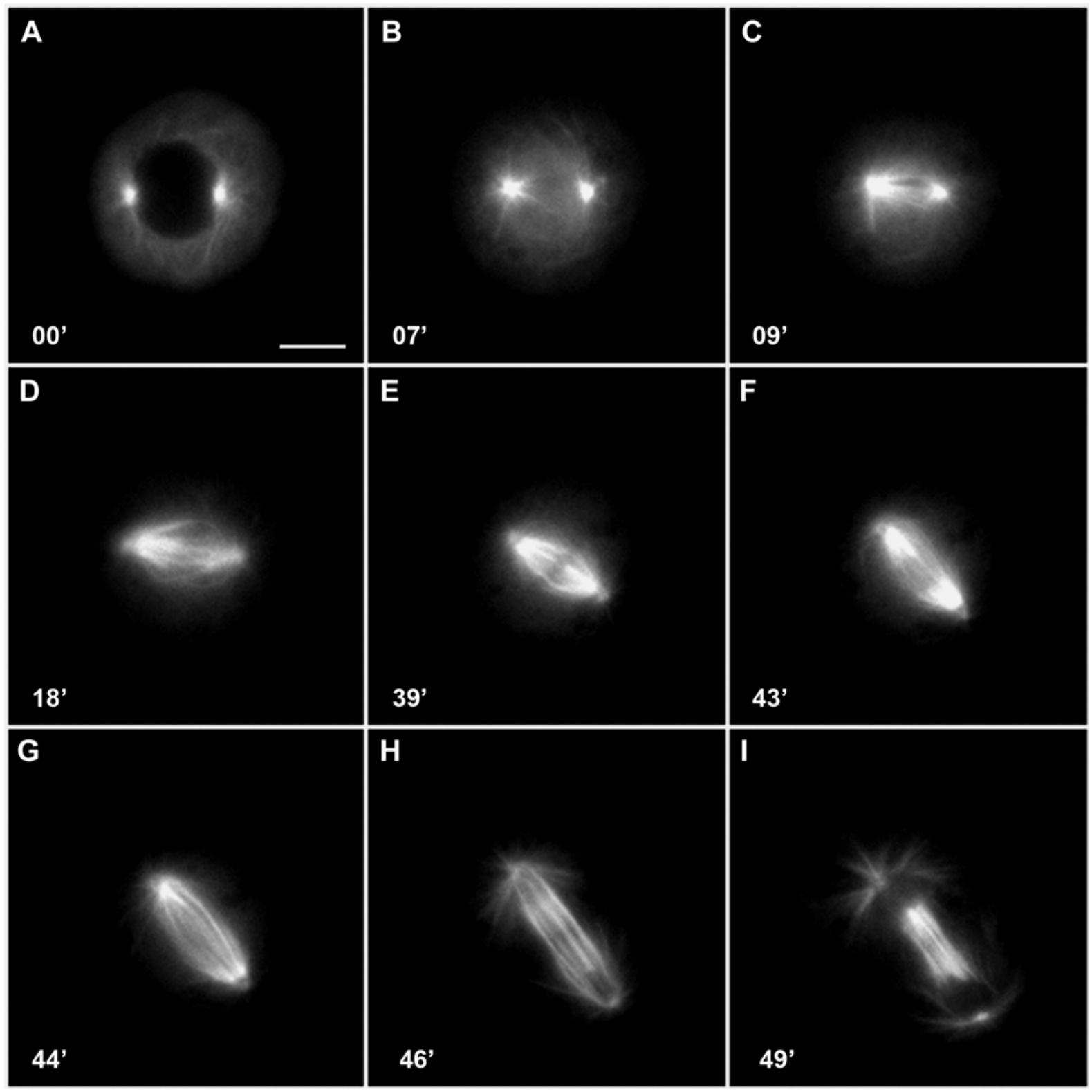

FIGURE 3 


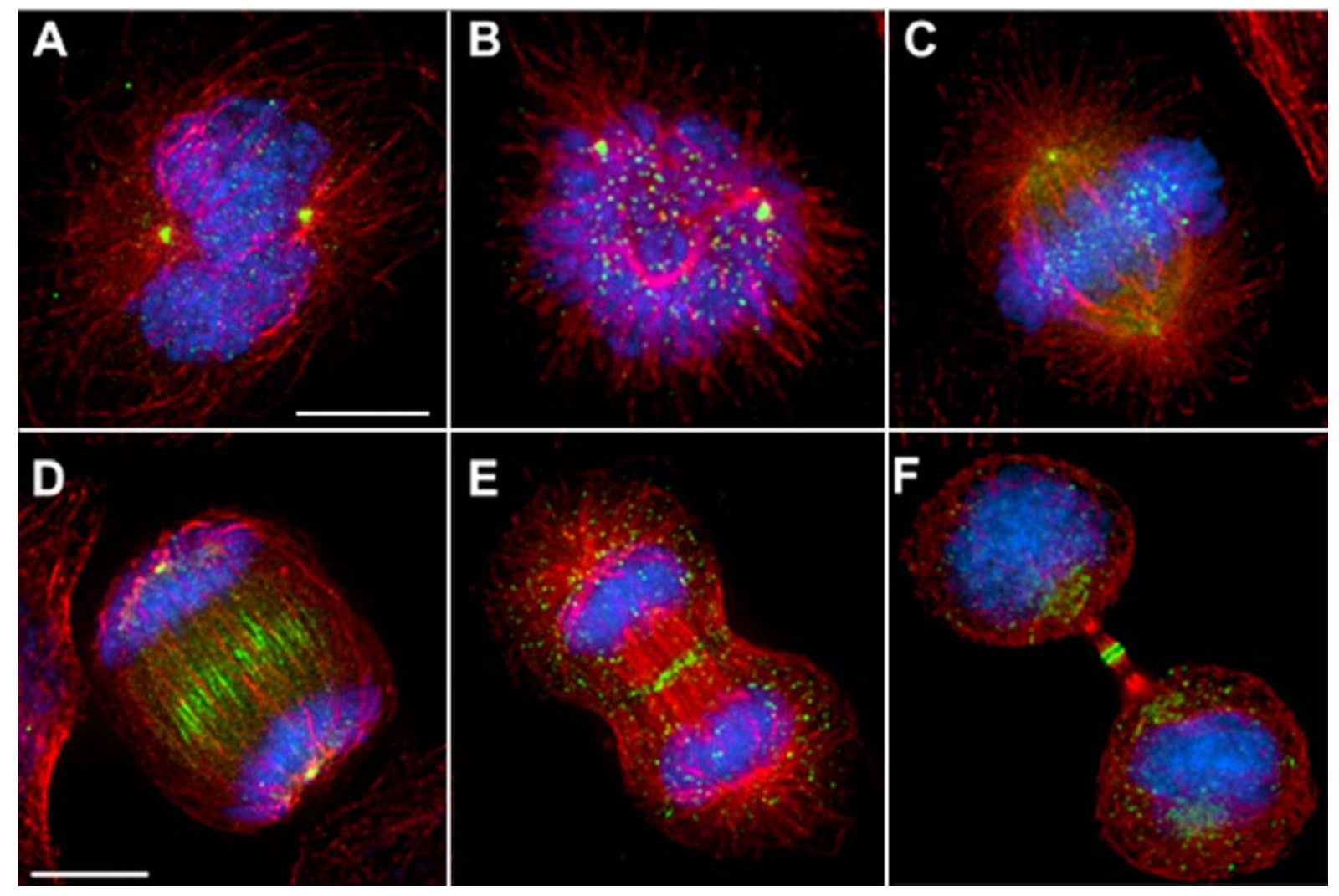

FIGURE 4 


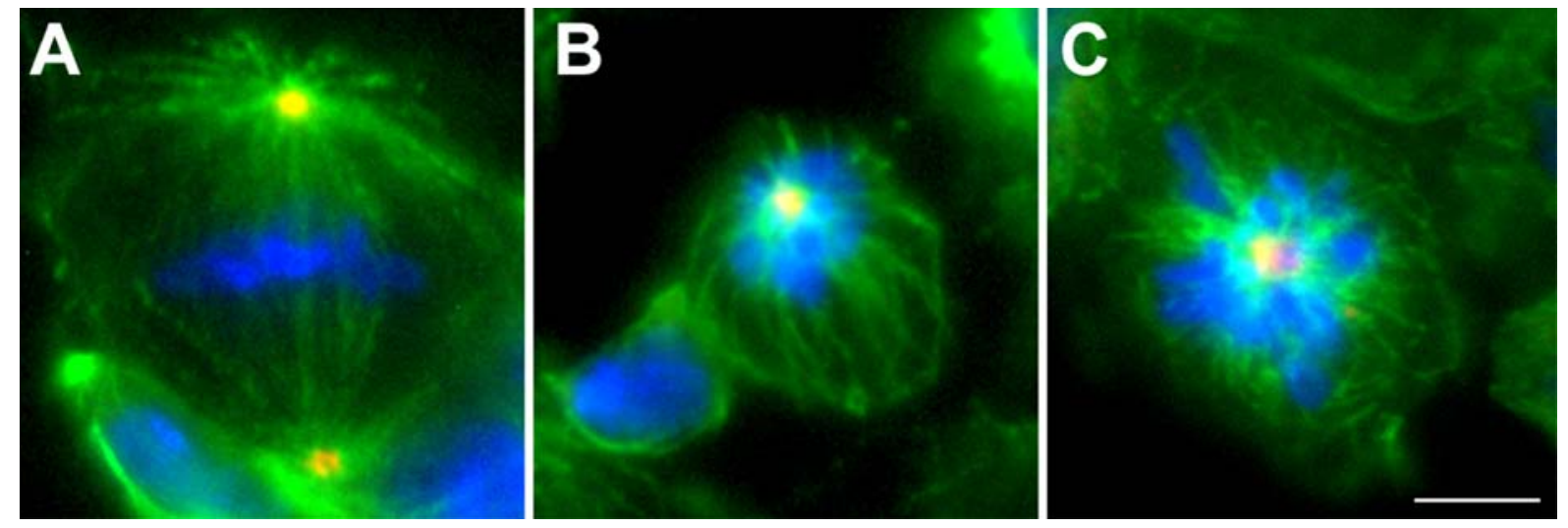

FIGURE 5 
A

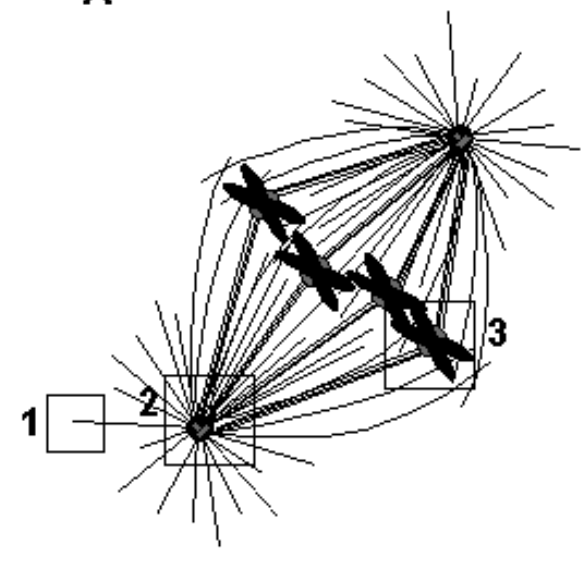

B

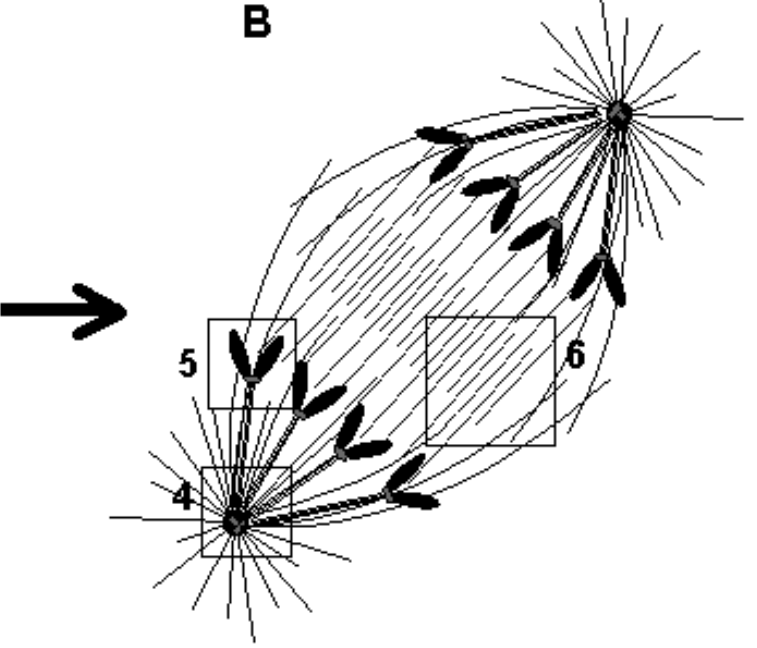

1. CLIP-170, Lis1, EB1, APC, CLASP, Dynein/Dynactin, CENP-E

2. CLASP, Dynein/Dynactin, NuMA, Kin I, EB1, APC, Dis-TOG, CLIP-170, C-KIN, TACC, TPX2, Katanin, MAP4, Polo, Aurona A

3. CLIP-170, CENP-E, KIN I, INCENP, Survivin, Aurora B, Dynein/Dynactin, Polo, Lis1, EB1, Chromokinesin, APC

4. CLASP, CENPE, KIN I, EB1, Dynactin/Dynein, Polo, Aurora A

5. CLASP, CENP-E, KIN I, Dynein/Dynactin

6. MKLP1, INCENP, Survivin, Aurora B, CENP-E, CLASP, PRC1, Polo 
Table 1. Families of microtubule associated proteins

\begin{tabular}{|c|c|c|c|c|c|}
\hline $\begin{array}{l}\text { MAP } \\
\text { Family }\end{array}$ & Members & Localization & Function & $\begin{array}{l}\text { Roles in } \\
\text { mitosis }\end{array}$ & References \\
\hline MAP4 & $\begin{array}{l}\text { MAP205 - Drosophila } \\
\text { XMAP230 - Xenopus } \\
\text { MAP4 - Mammalian }\end{array}$ & $\begin{array}{l}\text { Along interphasic and } \\
\text { mitotic MTs }\end{array}$ & MT stabilization & $\begin{array}{l}\text { Spindle organization } \\
\text { Chromosome } \\
\text { segregation }\end{array}$ & $\begin{array}{l}\text { Pereira et al., 1992; } \\
\text { Andersen et al., 1994; } \\
\text { Bulinski and Borisy, } \\
\text { 1980b; Bulinski and } \\
\text { Borisy, 1980c }\end{array}$ \\
\hline CLIP-170 & $\begin{array}{l}\text { Bik1 - S. cereviseae } \\
\text { Tip1 - S. pombe } \\
\text { D-CLIP-190 - Drosophila } \\
\text { CLIP-170 - Mammalian }\end{array}$ & $\begin{array}{l}\text { Plus ends of growing MTs } \\
\text { Prometaphase } \\
\text { kinetochores (Dynein } \\
\text { dependent) }\end{array}$ & MT stabilization & $\begin{array}{l}\text { Chromosome } \\
\text { congression } \\
\text { MT-Kinetochore } \\
\text { interaction }\end{array}$ & $\begin{array}{l}\text { Berlin et al., 1990; } \\
\text { Brunner and Nurse, 2000; } \\
\text { Lantz and Miller, 1998; } \\
\text { Pierre et al., 1992; } \\
\text { Rickard and Kreis, } 1990 . \\
\text { Review in Schuyler and } \\
\text { Pellman, 2001a }\end{array}$ \\
\hline CLASP & $\begin{array}{l}\text { Stu1 - S. cereviseae } \\
\text { MAST/Orbit - Drosophila } \\
\text { CLASP1, CLASP2 - } \\
\text { Mammalian }\end{array}$ & $\begin{array}{l}\text { Plus ends of growing MTs } \\
\text { Centrosomes } \\
\text { Spindle midzone } \\
\text { Mid-body } \\
\text { Kinetochores (MTs } \\
\text { independent) }\end{array}$ & MT stabilization & $\begin{array}{l}\text { Chromosome } \\
\text { congression } \\
\text { Kinetochore MT } \\
\text { dynamics Bipolar } \\
\text { spindle assembly } \\
\text { and maintenance }\end{array}$ & $\begin{array}{l}\text { Pasqualone and Huffaker, } \\
\text { 1994; Inoue et al., 2000; } \\
\text { Lemos et al., 2000; } \\
\text { Maiato et al., 2003a }\end{array}$ \\
\hline APC & $\begin{array}{l}\text { Kar9p - S. cereviseae } \\
\text { E-APC, APC2, DAPC - } \\
\text { Drosophila } \\
\text { APR-1 - C. elegans } \\
\text { APC - Xenopus } \\
\text { APC - Mammalian }\end{array}$ & $\begin{array}{l}\text { MT cytoskeleton } \\
\text { Plus ends of growing MTs } \\
\text { Kinetochores (MT } \\
\text { dependent) }\end{array}$ & $\begin{array}{l}\text { MT plus-ends } \\
\text { stabilization }\end{array}$ & $\begin{array}{l}\text { Chromosome } \\
\text { segregation } \\
\text { Interact with } \\
\text { kinetochore- } \\
\text { attachment } \\
\text { checkpoint proteins } \\
\text { Spindle positioning }\end{array}$ & $\begin{array}{l}\text { Miller et al., 2000; Miller } \\
\text { and Rose, 1998; Yu et al., } \\
\text { 1999; McCartney and al., } \\
\text { 1999; Hayashi et al., } \\
\text { 1997; Hoier et al., 2000; } \\
\text { Mimori-Kiyosue et al., } \\
\text { 2000a; Munemitsu et al., } \\
\text { 1994. Review in Mimori- } \\
\text { Kiyosue and Tsukita, } \\
\text { 2001; Bienz, } 2002\end{array}$ \\
\hline EB1 & $\begin{array}{l}\text { Bim1p - S.cereviseae } \\
\text { Mal3p - S. pombe } \\
\text { DdEB1 - Dictyostelium } \\
\text { dEB1 - Drosophila } \\
\text { EB1 - C. elegans } \\
\text { EB1 - Xenopus } \\
\text { EB1 - Mammalian } \\
\text { AtEB1 - Plant }\end{array}$ & $\begin{array}{l}\text { Along MTs with } \\
\text { accumulation on plus ends } \\
\text { of growing MTs } \\
\text { Centrosomes } \\
\text { Kinetochores (MT } \\
\text { dependent) }\end{array}$ & $\begin{array}{l}\text { MT stabilization } \\
\text { Promote MT plus- } \\
\text { ends dynamics }\end{array}$ & $\begin{array}{l}\text { Modulation of } \\
\text { kinetochore MTs } \\
\text { dynamics } \\
\text { Spindle assembly } \\
\text { and function } \\
\text { Spindle positioning }\end{array}$ & $\begin{array}{l}\text { Schwartz et al., 1997; } \\
\text { Beinhauer et al., 1997; } \\
\text { Rehberg and Graf, 2002; } \\
\text { Rogers et al., 2002; } \\
\text { Tirnauer and Bierer, } \\
\text { 2000; Tirnauer et al., } \\
\text { 2002b; Su et al., 1995; } \\
\text { Mathur et al., 2003. } \\
\text { Review in Tirnauer and } \\
\text { Bierer, } 2000\end{array}$ \\
\hline Lis1 & $\begin{array}{l}\text { Pac1p - S. cereviseae } \\
\text { NUDF- Aspergillus } \\
\text { nidulans } \\
\text { DmLis1 - Drosophila } \\
\text { Lis1 - Mammalian }\end{array}$ & $\begin{array}{l}\text { MT plus-ends } \\
\text { Kinetochores (Dynein } \\
\text { dependent) } \\
\text { Cell cortex }\end{array}$ & $\begin{array}{l}\text { Coordination between } \\
\text { motor activity and } \\
\text { cargo binding }\end{array}$ & $\begin{array}{l}\text { Nuclear migration } \\
\text { Chromosome } \\
\text { congression Spindle } \\
\text { positioning }\end{array}$ & $\begin{array}{l}\text { Geiser et al., 1997; Liu et } \\
\text { al., 1999; Reiner and al., } \\
\text { 1993; } \\
\text { Review in Vallee, et al. } \\
2001\end{array}$ \\
\hline Katanin & $\begin{array}{l}\text { MEI-1/MEI-2 - C. elegans } \\
\text { Katanin - Xenopus } \\
\text { Katanin - Mamalian } \\
\text { Katanin - Plant }\end{array}$ & $\begin{array}{l}\text { Centrosomes } \\
\text { Spindle poles }\end{array}$ & MT severing & $\begin{array}{l}\text { Promote MT } \\
\text { dynamics at } \\
\text { onset of mitosis } \\
\text { Spindle organization }\end{array}$ & $\begin{array}{l}\text { Srayko et al., 2000; } \\
\text { McNally and Thomas, } \\
\text { 1998; McNally and } \\
\text { Thomas, 1998; Stoppin- } \\
\text { Mellet et al., 2002 } \\
\text { Review in Quarmby, } 2000\end{array}$ \\
\hline $\begin{array}{c}\text { Op18/stathm } \\
\text { in }\end{array}$ & $\begin{array}{l}\text { OP18/ stathmin - } \\
\text { Drosophila } \\
\text { OP18/ stathmin - Xenopus } \\
\text { OP18/ stathmin - } \\
\text { Mammalian }\end{array}$ & Cytoplasm & $\begin{array}{l}\text { MT destabilization } \\
\text { Sequestration of } \\
\text { tubulin dimmers } \\
\text { Promote microtubule } \\
\text { plus-end catastrophes }\end{array}$ & $\begin{array}{l}\text { Down regulation is } \\
\text { essential } \\
\text { for G2/M transition } \\
\text { and spindle } \\
\text { assembly }\end{array}$ & $\begin{array}{l}\text { Belmont and Mitchison, } \\
1996 . \\
\text { Reviews in Andersen, } \\
\text { 2000; Cassimeris, 2002; } \\
\text { Walczak, } 2000\end{array}$ \\
\hline
\end{tabular}




\begin{tabular}{|c|c|c|c|c|c|}
\hline KinI & $\begin{array}{l}\text { Kip3 - S. cereviseae } \\
\text { Klp5/Klp6 - S. pombe } \\
\text { KLP10A, KLP59C - } \\
\text { Drosophila } \\
\text { XKCM1 - Xenopus } \\
\text { MCAK - Mammalian }\end{array}$ & $\begin{array}{l}\text { Centrosome } \\
\text { Spindle poles } \\
\text { Kinetochore/centromere }\end{array}$ & $\begin{array}{l}\text { Depolymerization of } \\
\text { MT ends }\end{array}$ & $\begin{array}{l}\text { Spindle assembly } \\
\text { Chromosome } \\
\text { congression } \\
\text { and segregation } \\
\text { Correction of } \\
\text { improper } \\
\text { MT-kinetochore } \\
\text { attachments } \\
\text { Polewards } \\
\text { microtubule flux }\end{array}$ & $\begin{array}{l}\text { Severin et al., 2001; } \\
\text { Garcia et al., 2002b; } \\
\text { Goshima et al., 2003; } \\
\text { Rogers et al., 2004; } \\
\text { Walczak et al., 1996; } \\
\text { Wordeman and } \\
\text { Mitchison, } 1995 \\
\text { Review in Walczak, } 2003\end{array}$ \\
\hline Dis1-Tog & $\begin{array}{l}\text { Stu2 - S. cerevispae } \\
\text { Dis1, Alp14 - S. Pombe } \\
\text { DdCP224 - Dictyostelium } \\
\text { Msps - Drosophila } \\
\text { Zyg9 - C. elegans } \\
\text { XMAP215 - Xenopus } \\
\text { ch-TOG - Mammalian } \\
\text { Mor1 - Plant }\end{array}$ & $\begin{array}{l}\text { Centrosome } \\
\text { Along interphase and } \\
\text { mitotic MTs } \\
\text { Accumulate at plus ends } \\
\text { of MTs } \\
\text { Kinetochore (MT } \\
\text { dependent except in Dis1) }\end{array}$ & MT dynamicity & $\begin{array}{l}\text { Spindle organization } \\
\text { Organization of } \\
\text { spindle poles } \\
\text { Chromosome } \\
\text { congression and } \\
\text { segregation } \\
\text { MT nucleation and } \\
\text { anchor } \\
\text { at the centrosome }\end{array}$ & $\begin{array}{l}\text { Wang and Huffaker, } \\
\text { 1997; Rockmill and } \\
\text { Fogel, 1988; Ohkura et } \\
\text { al., 1988; Garcia et al., } \\
\text { 2001; Graf et al., 2000; } \\
\text { Cullen et al., 1999; } \\
\text { Matthews et al., 1998; } \\
\text { Vasquez et al., 1994; } \\
\text { Charrasse et al., 1998; } \\
\text { Whittington et al., 2001 } \\
\text { Review in McNally, } 2003\end{array}$ \\
\hline TACC & $\begin{array}{l}\text { D-TACC - Drosophila } \\
\text { TAC-1 - C. elegans } \\
\text { TACC - Mammalian }\end{array}$ & Centrosomes & $\begin{array}{l}\text { Centrosome } \\
\text { localization of } \\
\text { Dis-tog proteins }\end{array}$ & $\begin{array}{l}\text { Organization of } \\
\text { spindle poles }\end{array}$ & $\begin{array}{l}\text { Gergely et al., 2000b; Le } \\
\text { Bot et al., 2003; Gergely } \\
\text { et al., 2000a; } \\
\text { Review in Gergely , } 2002\end{array}$ \\
\hline NUMA & $\begin{array}{l}\text { Asp - Drosophila } \\
\text { NUMA - Mammalian }\end{array}$ & Spindle poles & Crosslink MT fibers & $\begin{array}{l}\text { Bipolar spindle } \\
\text { organization Spindle } \\
\text { pole focusing }\end{array}$ & $\begin{array}{l}\text { Saunders et al., 1997a; } \\
\text { Lydersen and Pettijohn, } \\
1980 \\
\text { Review in Compton and } \\
\text { Clevland, } 1994\end{array}$ \\
\hline INCENP & $\begin{array}{l}\text { Sli15 - S. cereviseae } \\
\text { DmINCENP - Drosophila } \\
\text { CeINCENP - C. elegans } \\
\text { INCENP - Mammalian }\end{array}$ & $\begin{array}{l}\text { Chromosomes } \\
\text { Inner centromere } \\
\text { Spindle mid-zone } \\
\text { Cell cortex in the region } \\
\text { of contractile ring } \\
\text { formation }\end{array}$ & $\begin{array}{l}\text { Complex with } \\
\text { Survivin and Aurora } \\
\text { B } \\
\text { Localization and } \\
\text { regulation of Aurora } \\
\text { B }\end{array}$ & $\begin{array}{l}\text { Chromosome } \\
\text { congression } \\
\text { and segregation } \\
\text { Cytokinesis }\end{array}$ & $\begin{array}{l}\text { Kim, 1999; Adams et al., } \\
\text { 2001; Kaitna et al., 2000; } \\
\text { Cooke et al., } 1987 \\
\text { Review in Earnshaw, } \\
2001\end{array}$ \\
\hline Survivin & $\begin{array}{l}\text { Bir1p - S. cereviseae } \\
\text { Bir1 - S. pombe } \\
\text { BIR-1 - C. elegans } \\
\text { Survivin - Xenopus } \\
\text { Survivin - Mammalian }\end{array}$ & $\begin{array}{l}\text { Chromosomes } \\
\text { Inner centromere } \\
\text { Spindle mid-zone } \\
\text { Cell cortex in the region } \\
\text { of contractile ring } \\
\text { formation }\end{array}$ & $\begin{array}{l}\text { Complex with } \\
\text { INCENP and Aurora } \\
\text { B } \\
\text { Localization and } \\
\text { regulation of Aurora } \\
\text { B }\end{array}$ & $\begin{array}{l}\text { Chromossome } \\
\text { congression } \\
\text { and segregation } \\
\text { Cytokinesis } \\
\text { Kinetochore- } \\
\text { attachment } \\
\text { checkpoint response }\end{array}$ & $\begin{array}{l}\text { Yoon and Carbon, 1999; } \\
\text { Uren et al., 1999; Fraser } \\
\text { et al., 1999; Bolton et al., } \\
\text { 2002; Ambrosini et al., } \\
\text { 1997; Uren et al., } 2000 \\
\text { Review in Reed and } \\
\text { Bischoff, } 2000\end{array}$ \\
\hline Aurora & $\begin{array}{l}\text { Ipl1p - S. cereviseae } \\
\text { AuroraA, AuroraB - } \\
\text { Drosophila } \\
\text { AIR-1, AIR-2 - C. elegans } \\
\text { Eg2, XAIRK2 - Xenopus } \\
\text { Aurora A, Aurora B - } \\
\text { Mammalian }\end{array}$ & $\begin{array}{l}\text { Aurora A: } \\
\text { Centrosome } \\
\text { Aurora B: } \\
\text { Chromosomes } \\
\text { Inner centromere } \\
\text { Spindle mid-zone } \\
\text { Cell cortex in the region } \\
\text { of contractile ring } \\
\text { formation }\end{array}$ & Regulatory kinase & $\begin{array}{l}\text { Aurora A: } \\
\text { Centrosome } \\
\text { maturation } \\
\text { Bipolar spindle } \\
\text { organization } \\
\text { and function } \\
\text { Aurora B: } \\
\text { Chromosome } \\
\text { congression } \\
\text { and segregation } \\
\text { Cytokinesis } \\
\text { Targeting Kt } \\
\text { proteins }\end{array}$ & $\begin{array}{l}\text { Francisco et al., 1994; } \\
\text { Glover et al., 1995; Giet } \\
\text { and Glover, 2001; } \\
\text { Schumacher et al., 1998a; } \\
\text { Schumacher et al., 1998b; } \\
\text { Roghi et al., 1998; Adams } \\
\text { et al., 2000 } \\
\text { Review in Giet and } \\
\text { Prigent, 1999b; Carmena } \\
\text { and Earnshaw, } 2003\end{array}$ \\
\hline TPX2 & $\begin{array}{l}\text { TPX2 - Xenopus } \\
\text { hTPX2 - Mammalian }\end{array}$ & $\begin{array}{l}\text { Spindle poles } \\
\text { Spindle mid-zone and } \\
\text { mid-body }\end{array}$ & $\begin{array}{l}\text { Aurora A activation } \\
\text { and localization on } \\
\text { MTs }\end{array}$ & $\begin{array}{l}\text { Bipolar spindle } \\
\text { assembly } \\
\text { Spindle pole } \\
\text { organization }\end{array}$ & $\begin{array}{l}\text { Wittmann et al., 2000; } \\
\text { Garrett et al., 2002; }\end{array}$ \\
\hline
\end{tabular}




\begin{tabular}{|c|c|c|c|c|c|}
\hline $\begin{array}{l}\text { Cytoplasmat } \\
\text { ic Dynein }\end{array}$ & $\begin{array}{l}\text { Dyn1 - S. cereviseae } \\
\text { Dynein heavy chain - S. } \\
\text { pombe } \\
\text { NudA - Aspergillus } \\
\text { nidulans } \\
\text { Dhc64 - Drosophila } \\
\text { Dynein heavy chain - C. } \\
\text { elegans } \\
\text { Dynein heavy chain - } \\
\text { Xenopus } \\
\text { Dynein heavy chain - } \\
\text { Mammalian } \\
\text { Dynein heavy chain - Plant }\end{array}$ & $\begin{array}{l}\text { Cell cortex } \\
\text { Nuclear envelope } \\
\text { Kinetochore } \\
\text { Spindle poles } \\
\text { Plus-end of astral MTs }\end{array}$ & $\begin{array}{l}\text { Minus-end directed } \\
\text { motor }\end{array}$ & $\begin{array}{l}\text { Nuclear migration } \\
\text { Anchor astral MTs } \\
\text { to cell cortex } \\
\text { Centrosome } \\
\text { separation } \\
\text { Bipolar spindle } \\
\text { organization } \\
\text { Organization of } \\
\text { spindle poles } \\
\text { Poleward } \\
\text { chromosome } \\
\text { movement } \\
\text { Spindle positioning } \\
\text { Inactivation of } \\
\text { kinetochore- } \\
\text { attachment } \\
\text { checkpoint }\end{array}$ & $\begin{array}{l}\text { Lye et al., 1987; Paschal } \\
\text { et al., 1987. Reviews in } \\
\text { Hirokawa et al., 1998; } \\
\text { Karki and Holzbaur, } \\
\text { 1999; Schroer, 1994; } \\
\text { Vallee and Gee, 1998; }\end{array}$ \\
\hline CENP-E & $\begin{array}{l}\text { DmCmeta - Drosophila } \\
\text { CENP-E - Xenopus } \\
\text { CENP-E - Mammalian } \\
\text { Cpe12 - Plant }\end{array}$ & $\begin{array}{l}\text { Kinetochore } \\
\text { Spindle mid-zone and } \\
\text { mid-body }\end{array}$ & $\begin{array}{l}\text { Plus-end directed } \\
\text { motor }\end{array}$ & $\begin{array}{l}\text { MT capture by } \\
\text { kinetochore } \\
\text { Kinetochore } \\
\text { attachment to } \\
\text { depolymerizing MTs } \\
\text { Chromosome } \\
\text { congression } \\
\text { Interact with } \\
\text { Kinetochore- } \\
\text { attachment } \\
\text { checkpoint proteins }\end{array}$ & $\begin{array}{l}\text { Yucel et al., 2000; Wood } \\
\text { et al., 1997; Yen et al., } \\
1991 ; \text { ten Hoopen et al., } \\
2002 \\
\text { Review in Grancell and } \\
\text { Sorger, } 1998\end{array}$ \\
\hline $\begin{array}{l}\text { Bipolar } \\
\text { Kinesin }\end{array}$ & $\begin{array}{l}\text { Cin8, Kip1 - S. cereviseae } \\
\text { Cut7 - S. pombe } \\
\text { KLP61F - Drosophila } \\
\text { Eg5 - Xenopus } \\
\text { HsEg5 - Mammalian }\end{array}$ & Spindle MT & $\begin{array}{l}\text { Plus-end directed } \\
\text { motor } \\
\text { Sliding anti-parallel } \\
\text { MTs }\end{array}$ & $\begin{array}{l}\text { Bipolar spindle } \\
\text { organization }\end{array}$ & $\begin{array}{l}\text { Hoyt et al., 1992; Roof et } \\
\text { al., 1992; Hagan and } \\
\text { Yanagida, 1990; Barton et } \\
\text { al., 1995; Heck et al., } \\
\text { 1993; Le Guellec et al., } \\
\text { 1991; Sawin et al., 1992; } \\
\text { Blangy et al., 1995 } \\
\text { Review in Kashina et al } \\
1997\end{array}$ \\
\hline $\begin{array}{l}\text { C-terminal } \\
\text { Kinesin }\end{array}$ & $\begin{array}{l}\text { Kar3p - S. cereviseae } \\
\text { Ncd - Drosophila } \\
\text { XCTK2 - Xenopus } \\
\text { HSET - Mammalian }\end{array}$ & Spindle MT & $\begin{array}{l}\text { Minus-end directed } \\
\text { motor }\end{array}$ & $\begin{array}{l}\text { Bipolar spindle } \\
\text { organization Spindle } \\
\text { positioning }\end{array}$ & $\begin{array}{l}\text { Middleton and Carbon, } \\
\text { 1994; McDonald et al., } \\
\text { 1990; Walczak et al., } \\
\text { 1997; Ando et al., 1994; } \\
\text { Kuriyama et al., 1995; }\end{array}$ \\
\hline \begin{tabular}{|} 
Chromokine \\
$\sin$
\end{tabular} & $\begin{array}{l}\text { Nod, KLP38B, KLP3A - } \\
\text { Drosophila } \\
\text { Xklp1, Xkid1 - Xenopus } \\
\text { Kid, KIF4 - Mammalian }\end{array}$ & $\begin{array}{l}\text { Chromosome arms } \\
\text { Spindle mid-zone and } \\
\text { mid-body }\end{array}$ & $\begin{array}{l}\text { Plus-end directed } \\
\text { motor }\end{array}$ & $\begin{array}{l}\text { Spindle organization } \\
\text { Polar injection } \\
\text { forces Chromosome } \\
\text { congression }\end{array}$ & $\begin{array}{l}\text { Afshar et al., 1995a; } \\
\text { Vernos et al., 1995; } \\
\text { Antonio et al., 2000; } \\
\text { Funabiki and Murray, } \\
\text { 2000; Levesque and } \\
\text { Compton, 2001; Tokai et } \\
\text { al., 1996; Lee et al., } \\
\text { 2001b; }\end{array}$ \\
\hline MKLP1 & $\begin{array}{l}\text { Pavarotti - Drosophila } \\
\text { ZEN-4, CeMKLP1 - C. } \\
\text { elegans } \\
\text { CHO1, MKLP1 - } \\
\text { Mammalian }\end{array}$ & Spindle mid-zone & $\begin{array}{l}\text { Plus-end directed } \\
\text { motor }\end{array}$ & Cytokinesis & $\begin{array}{l}\text { Adams et al., 1998; } \\
\text { Williams et al., 1995; } \\
\text { Raich et al., 1998; Powers } \\
\text { et al., 1998; Nislow et al., } \\
\text { 1992; Sellitto and } \\
\text { Kuriyama, 1988; }\end{array}$ \\
\hline PRC1 & $\begin{array}{l}\text { Ase1p - S. cereviseae } \\
\text { PRC1 - Mammalian }\end{array}$ & Spindle mid-zone & MT bundling & $\begin{array}{l}\text { Anaphase B } \\
\text { Cytokinesis }\end{array}$ & $\begin{array}{l}\text { Pellman et al., 1995; Jiang } \\
\text { et al., 1998; }\end{array}$ \\
\hline Polo & $\begin{array}{l}\text { cdc5p - S. cereviseae } \\
\text { Plo1 - S. pombe } \\
\text { Polo - Drosophila } \\
\text { Plx1 - Xenopus } \\
\text { Plk1 - Mammalian }\end{array}$ & $\begin{array}{l}\text { Centrosome, Kinetochore, } \\
\text { Spindle mid-zone } \\
\text { Mid-body }\end{array}$ & Regulatory kinase & $\begin{array}{l}\text { Centrosome } \\
\text { maturation } \\
\text { G2/M transition } \\
\text { Spindle organization } \\
\text { Activation of } \\
\text { anaphase-promoting } \\
\text { complex } \\
\text { Cytokinesis }\end{array}$ & $\begin{array}{l}\text { Kitada et al., 1993; } \\
\text { Ohkura et al., 1995; } \\
\text { Llamazares et al., 1991; } \\
\text { Sunkel and Glover, 1988; } \\
\text { Kumagai and Dunphy, } \\
\text { 1996; Golsteyn et al., } \\
\text { 1995; } \\
\text { Review in Glover et al. } \\
1998\end{array}$ \\
\hline
\end{tabular}


